\title{
A Bird's-Eye View: \\ Core Knowledge in wild North Island Robins of New Zealand
}

By

Alexis Garland

\begin{abstract}
A thesis
Submitted to the Victoria University of Wellington

in fulfilment of the requirements for the degree of

Doctor of Philosophy

in Psychology
\end{abstract}

Victoria University of Wellington

2013 
This is dedicated to

Dr. Louie Arnold Dow

25 April 1927 - 5 February 2013

who stuck with me right through to my defense before gently leaving us, and my Marley, whose Butterbean I will always be.

To

\section{Dr. Sandra Michalik}

the teacher who always had faith that I would finish my homework eventually, and who let me hold her newly printed dissertation in 5th grade;

something I have never forgotten.

Of course, to my mother,

Dr. Shannan McNair,

who I watched walk across the stage for her own Doctoral achievement and whose dissertation I have many early memories of.

To my father,

Dr. Frank Garland,

who always nurtured my intense curiosity and note-taking,

and who I will continue look around for when hearing my newly minted title.

And to my brother,

Colin Garland

who came along to the other side of the world with me, and kept life exciting. 


\begin{abstract}
A prominent psychological theory on early cognitive development is Spelke's Core Knowledge (CK) hypothesis (Spelke, Breinlinger, Macomber, \& Jacobson, 1992), which posits that human infants, and possibly other species, are guided by innate understandings of how object movements, classification and quantification are governed by physical laws and, further, how agents are capable of perceptions and purposive action. $\mathrm{CK}$ is a set of cognitive building blocks, which serve as the foundation for more complex cognition such as acquisition and use of symbol systems pertaining to language and mathematics (Spelke, 2000). Evidence points to four core systems of knowledge: representation of number, object, space (or geometry) and agency. Investigation of spontaneous $\mathrm{CK}$ in nonhuman species in the wild is fundamental to understanding the ecological validity and evolutionary context for a set of systems that is proposed to be universally embedded. The bold, inquisitive manner, naïve fearlessness and unique insect caching behaviour of wild North Island robins (Petroica longipes) presents a unique opportunity to identify and characterise CK in a new model system. Six studies were conducted with the aim of investigating core developmental cognition in robins.

The first three studies focused on perception of numerical quantity. Study 1 investigated the ability to discriminate between both large and small quantities, finding that robins successfully discriminate between unusually large quantities independent of ratio. Study 2 explored quantity discrimination in which summation of items is spatially distributed across an array, and found that while robins perform successfully with small numbers, the task presented substantially more cognitive demand. Study 3 measured robins' reactions to computation by presenting simple addition and subtraction problems in a Violation of Expectancy (VoE) paradigm, finding that robins search longer when presented with a mathematically incongruent scenario.

The last three studies focused on perception of agency. Study 4 investigated robins' response to gaze direction in humans in a competitive paradigm, and found that they were sensitive to human gaze direction in all conditions but one. Study 5 explored perception of physical capability in humans, and results indicated that limb visibility significantly influences pilfering choice. Study 6 examined robins' perception of animacy in prey, finding that in a VoE paradigm, robins' expectation of hidden prey continuity varies depending on mobility and animacy.

Taken together, the results of these six studies suggest that while supportive of fundamental characteristics defining basic Core Knowledge in many ways, some unique results in the cognitive abilities of this biologically naïve species shed new light on our growing understanding of the shared basis of cognition. A deeper look at avian performance in core developmental tasks, especially in a naïve wild population, can offer new insights into sweeping evolutionary theories that underpin basic cognitive mechanisms.
\end{abstract}




\section{Acknowledgments}

A debt of gratitude is owed to all of the kind, patient, inspirational, persistent and intelligent people that surround and support me. The time I have spent living, studying and experiencing life as a PhD student in Wellington, New Zealand have been several of the most unforgettable years of my existence, and it is with great reluctance that I bring this experience to a close.

First and foremost, I owe eternal thanks to my advisors. Doctoral advisors are sometimes called 'Doktorvater' in German, which translates literally to 'doctoral father,' and this is precisely what my advisors were to me: my academic parents. I feel that they invested in me not only as a student, but as a person - and I have come away changed. Without Jason's guidance, kindness, and conviction in my abilities, I would almost certainly have emerged less bravely from this adventure. The endless detailed hours he spent on aiding both my project and myself as a researcher have left me continuing to strive for ways to improve as a learner. It is KC who answered the fateful message that led me to New Zealand in the first place and it is his continued enthusiasm for the field and belief in me that provided such essential support along the path.

Sanctuary staff and volunteers are and continue to be an essential cog in the wheel of conserving New Zealand wildlife. It is their endless work, on an unimaginable scale, which makes theoretical work like mine possible. I was lucky enough to work with and learn from a handful of Zealandia colleagues who are an endless well of knowledge about New Zealand forests and the native winged inhabitants therein. They maintain a level of passion about their work that I both admire and appreciate the results of - restoration.

Family and friends are those to whom I have always been and will be ever thankful for the support of. Especially without my mother's unswerving support and unwavering persistence, I would never have arrived at this opportunity to begin with - and it is only the staunch support of her, my family, my adoptive 'Kiwi' family in New Zealand, and the network of close friends all behind me that such an endeavour could be undertaken or achieved.

Finally, I would, of course, like to thank the ones without whom this dissertation would not have existed - the birds. Sitting in the forest and learning new things while I observed their antics in the world around them, I continually came only to a single conclusion: I must be the luckiest person on earth.

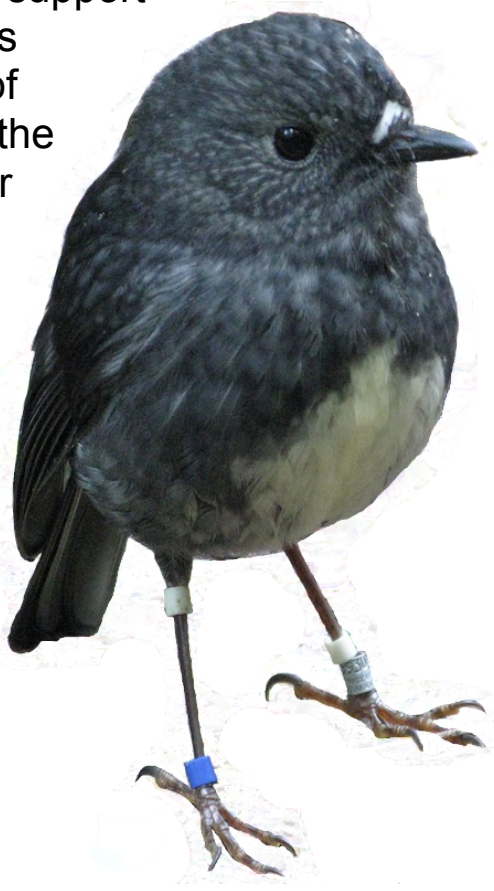




\section{Table of Contents}

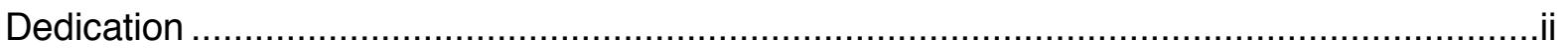

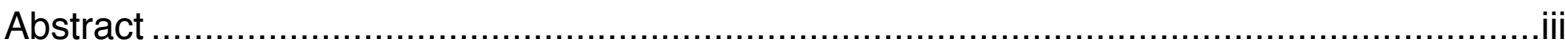

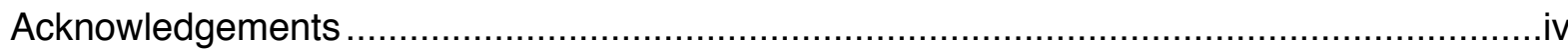

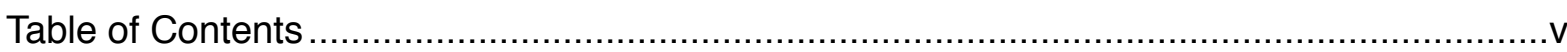

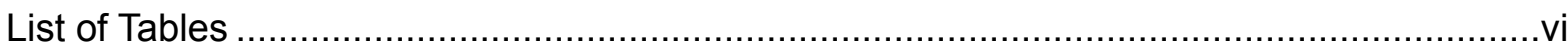

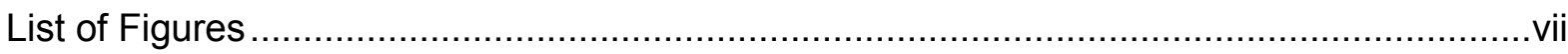

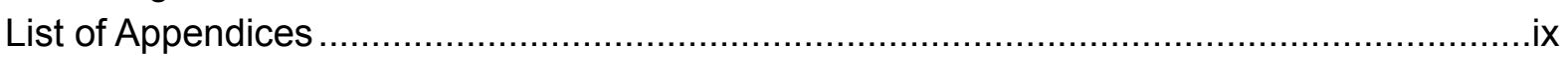

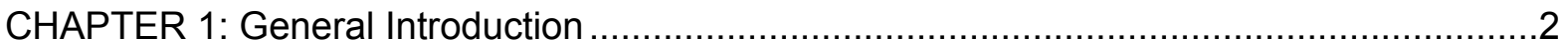

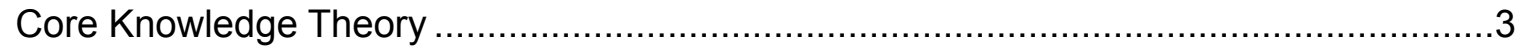

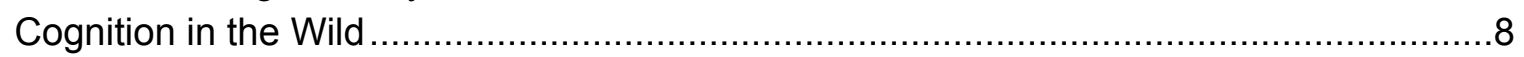

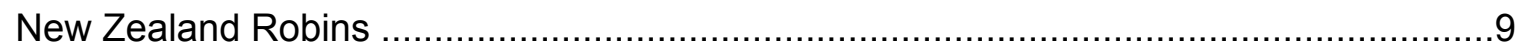

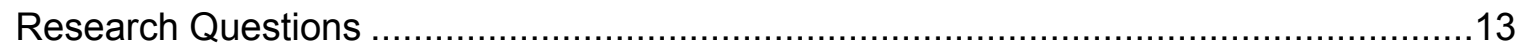

CHAPTER 2: Study 1 - Discrimination of Large and Small Quantities ...............................14

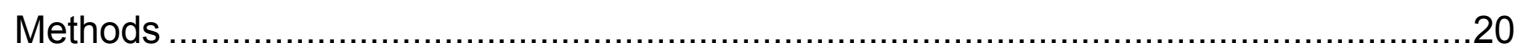

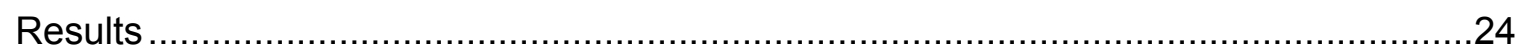

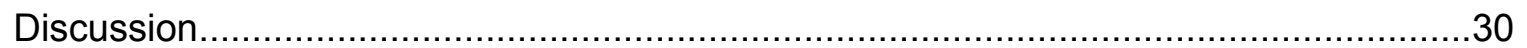

CHAPTER 3: Study 2 - Quantity Discrimination Across Multiple Arrays ...............................34

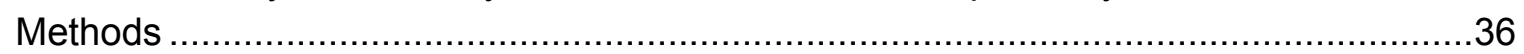

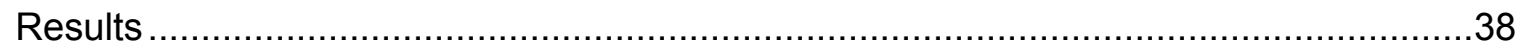

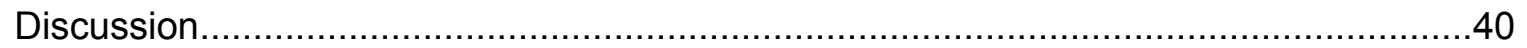

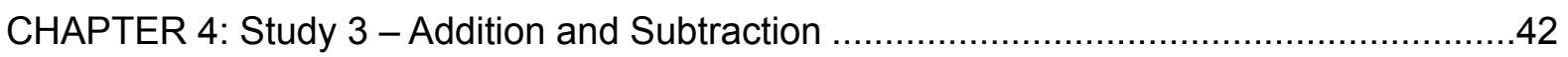

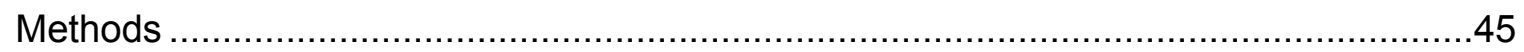

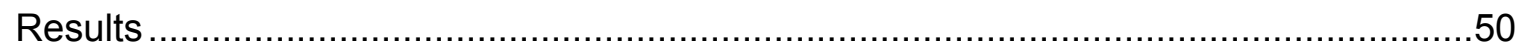

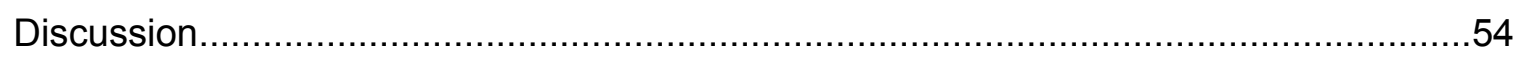

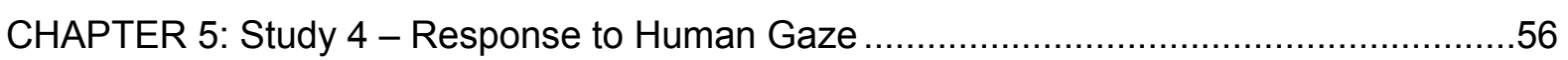

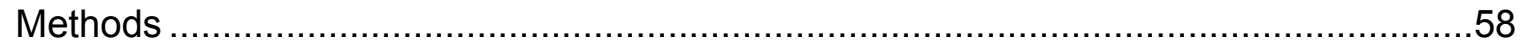

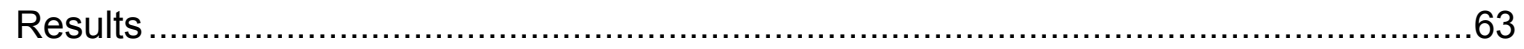

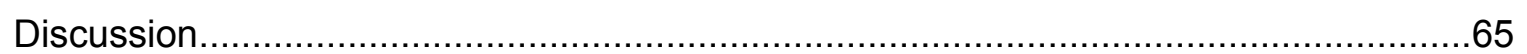

CHAPTER 6: Study 5 - Response to Human Physical Capability ...................................... 70

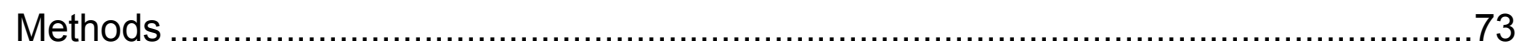

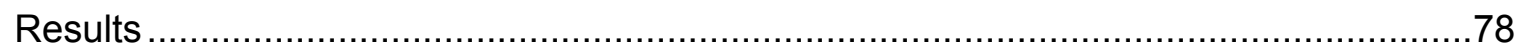

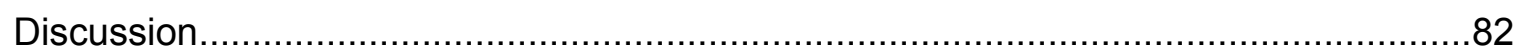

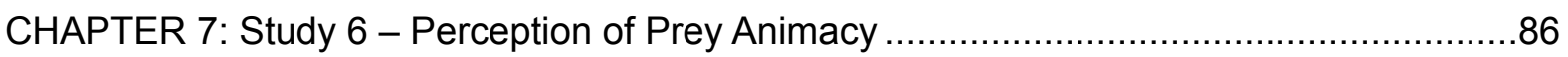

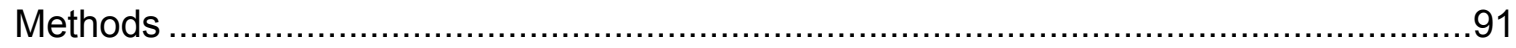

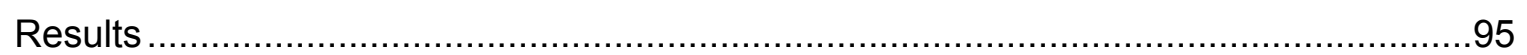

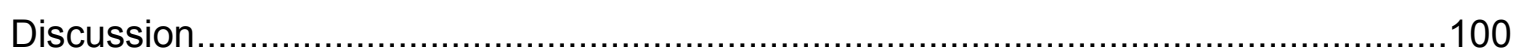

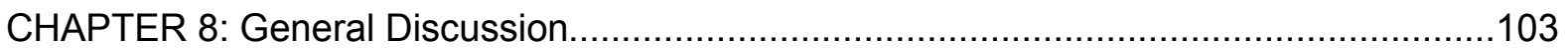

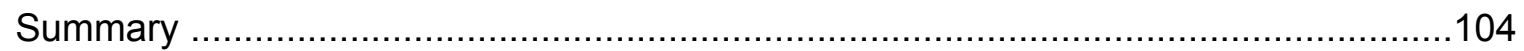

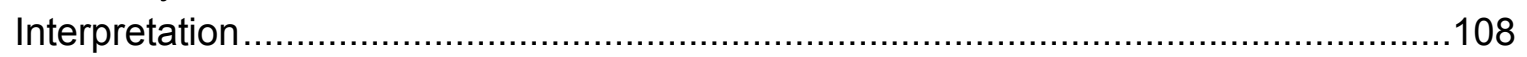

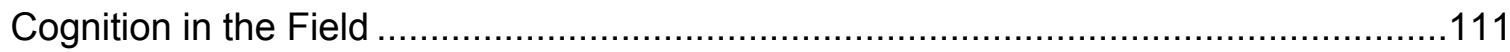

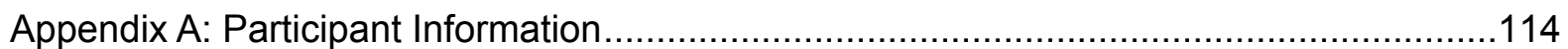

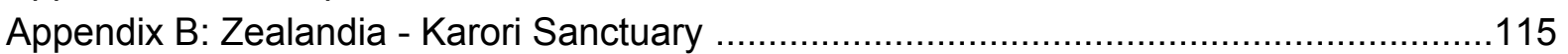

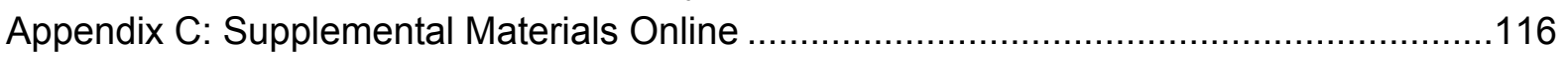

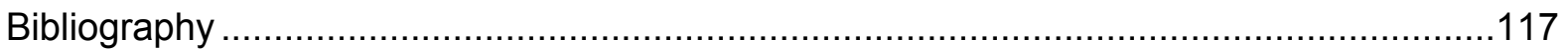




\section{List of Tables}

Table 1. Number comparisons and total number of worms displayed for each trial for the 5 ratios investigated across Experiments 1,2 and 3.

Table 2. Percentage of correct trials across Experiments 1, 2 and 3 in both 'small' and 'large' comparisons.

Table 3. Congruent and Incongruent conditions presented in Experiment 1, altering item number

Table 4. Congruent and Incongruent conditions presented in Experiment 2, altering item category, where ' $m$ ' is mealworm, and ' $w$ ' is waxworm.

Table 5. Congruent and Incongruent conditions presented in Experiment 1, altering item type.

Table 6. Congruent and Incongruent conditions presented in Experiment 2, altering item animacy. 


\section{List of Figures}

Figure 1. An adult mated pair of North Island robins (Petroica longipes)

Figure 2. A map of robin territories in one portion of the sanctuary

Figure 3. A robin makes his choice in a sequential number task.

Figure 4. Percentage of correct trials as a function of (a) ratio and (b) total number of worms (combined set size), for item-by-item presentation of comparisons containing up to 30 total prey items $(\mathrm{N}=12)$.

Figure 5. Percentage of correct trials as a function of (a) ratio and (b) total number of worms (combined set size), for whole set presentation of comparisons containing up to 30 total prey items $(\mathrm{N}=12)$.

Figure 6. Percentage of correct trials as a function of (a) ratio and (b) total number of worms (combined set size), for whole set presentation of comparisons containing up to 120 total prey items $(\mathrm{N}=12)$

Figure 7. Percentage of correct trials as a function of the larger number of worms of the two quantities shown in trials of Experiments 2 and 3.

Figure 8. A robin makes a choice between two arrays

Figure 9. Percentage success across all participants for all 6 number comparisons, overall, in winter, and in summer.

Figure 10. The VoE apparatus: Out of view of the bird, the upper compartment is slid in, (a) insects placed inside and (b) the compartment is slid out, hiding it. In view of the bird, in the lower compartment (c) insects are placed inside, (d) the lid covers the well, (e) the drawer is slid in, and (f) the bird uncovers and accesses the upper compartment.

Figure 11. Experiment 1 (Item number) - Average response in search and attendance time and number of pecks.

Figure 12. Experiment 2 (Item category) - Average response in search and attendance time and number of pecks. 
Figure 13. Overall response by prey destination - Average response in search and attendance time and number of pecks.

Figure 14. Two experimenters in position during a trial of Condition 5.

Figure 15. The six conditions presented to robins. In each, one experimenter has full view of the mealworm in front of her, and the view of the other is obscured

Figure 16. Results for all six conditions, displaying number of subjects pilfering from each experimenter, with one trial per condition per subject.

Figure 17. The six conditions presented to robins in Experiment 1, using opaque brown cloth to obscure the body or limbs of the experimenters.

Figure 18. The six conditions presented to robins in Experiment 2, using a wooden plank to obscure the body or limbs of the experimenters.

Figure 19. Results for all six conditions in Experiment 1, displaying number of subjects pilfering from each experimenter, with one trial per condition per subject...79

Figure 20. Results for all four conditions in Experiment 2, displaying number of subjects pilfering from each experimenter, with one trial per condition per subject...81

Figure 21. The VoE apparatus: Out of view of the bird, in the upper compartment, (a) insects are placed inside and (b) the compartment is slid out, hiding it. In view of the bird, in the lower compartment (c) insects are placed inside (d) the lid covers the well, (e) the drawer is slid in, and (f) the bird allowed to access the upper compartment.

Figure 22. Experiment 1 (Prey type) - Average response in search and attendance time and number of pecks.

Figure 23. Experiment 1 (Prey type) - Average response in search and attendance time and number of pecks by individual insect.

Figure 24. Experiment 2 (Prey animacy) - Average response in search and attendance time and number of pecks.

Figure 25. Overall response by prey destination - Average response in search and attendance time and number of pecks. 100 


\section{List of Appendices}

Appendix A. Information about experimental participation, approximate age, and sex of North Island robins observed interacting in these studies. Approximate current age is provided; age at testing varied based on date of data collection. .....................114

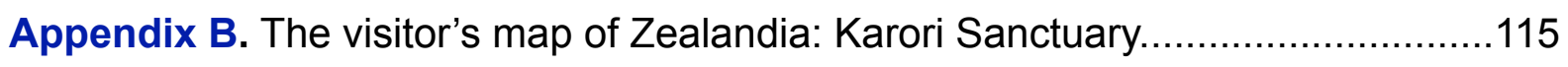

Appendix C. Supplemental Materials Online - Sample Trial Videos. ...................116 
"Now listen, Doctor, and I'll tell you something. Did you know animals can talk?" "My! You don't say so!" said the Doctor. "You never talked that way to me before." "What would have been the good?" said Polynesia. "You wouldn't have understood me if I had."

- Hugh Lofting, Doctor Dolittle 1 
CHAPTER 1: General Introduction 
While there is a clear divide between human language and cognition and the communicative and cognitive systems by which other species function, research has yet to fully connect the dots to create a clear picture of just what that gap might comprise. Even less is known about how those systems found in animals develop and function in their natural state.

\section{Core Knowledge Theory}

A prominent psychological theory on early cognitive development is Spelke's Core Knowledge (CK) hypothesis (Spelke et al., 1992), which posits that human infants, and possibly other species, are guided by innate understandings of how object movements, classification and quantification are governed by physical laws and, further, how agents are capable of perceptions and purposive action. CK is a set of cognitive building blocks, which serve as the foundation for more complex cognition such as acquisition and use of symbol systems pertaining to language and mathematics (Spelke, 2000).

Evidence points to four core systems of knowledge: representation of number, object, space (or geometry) and agency. Each system is defined by a set of cognitive signatures (sometimes referred to as "constraints" or "limits") that identifies its functioning in tasks across variables such as age, gender, species or culture (Spelke \& Kinzler, 2007). Research suggests that these core systems are domain specific, task specific, and relatively encapsulated and automatic (not susceptible to explicit belief reasoning) (Von Bayern \& Emery, 2009). In spite of the proliferation of evidence on CK in the human and nonhuman primate lineage (Hanus \& Call, 2007; Hauser, MacNeilage, \& Ware, 1996), a detailed understanding of precise way in which these core systems manifest themselves in other species of nonhuman animals bears continued exploration. Furthermore, investigation of spontaneous CK in the wild is fundamental to understanding the ecological validity and evolutionary context for a set of systems that is proposed to be universally embedded (Emery et al., 2005).

To date, establishing both experimental and ethological significance of CK has been rarely achieved because researchers face significant logistical difficulties working with wild animals as compared to trained, captive subjects 
(McComb, Packer, \& Pusey, 1994). The bold, inquisitive manner and naïve fearlessness of wild North Island robins (Petroica longipes) presents a unique opportunity to identify and characterise $\mathrm{CK}$ in a new model system (Menzies \& Burns, 2008).

A cognitive domain represents a given system operating within a finite, contextspecific computational problem space, in which a psychological mechanism only becomes engaged when faced with specific types of scenarios. Each system operates by picking out certain relevant 'signature characteristics' (and rejecting others) using specialised learning mechanisms (Flombaum, Santos, \& Hauser, 2002).

Number representation. With respect to number, several competing proposed paradigms have postulated differing sets of principles to define this system (Changeux \& Dehaene, 1989; Church \& Broadbent, 1990; Meck \& Church, 1983). However, at least two basic models have been postulated among others; a single continuum of representation, subject to scalar variability (Cordes, Gelman, Gallistel, \& Whalen, 2001), and a dual mechanism system operating object file/subitizing system for small numbers, and a magnitude representation system for larger numbers (Dehaene, Dehaene-Lambertz, \& Cohen, 1998). The object-file system computes numerical differences on the basis of one-to-one correspondence, but there appears to be a capacity limit of 3 to 4 objects to which file tokens may be assigned at any one time (Trick \& Pylyshyn, 1994). Performance in situations that exceed the limits of object-tracking system (e.g., discriminating 16 from 32 vs. 16 from 24) show that human infants and primates' numerosity judgments display the Weber-fraction signature of scalar variance. The cognitive signature of the magnitude representation system is that the ratio of two values determines discriminability.

Despite disagreement on the larger set of principles defining the domain of number, or precise deployment of multiple mechanisms, there is consensus over three signature features of number representation. First, number representations are imprecise in nature, and noise increases in a linear manner with cardinal value, and a ratio limit appears as a result of scalar variability which serve to constrain discriminability of sets containing different values (Gallistel \& Gelman, 2000; Izard \& Dehaene, 2008). Second, addition and subtraction can be used to compare and 
combine number representations (Brannon, Wusthoff, Gallistel, \& Gibbon, 2001). Third, number representations are abstract regardless of item characteristics: they apply to any range of articles experienced through multiple sensory inputs, including auditory sequences, visual arrays of objects or light, and perceived or sequences of motion or action. Pigeons and primates, for example, can both acquire abstract numerical rules such as ascending ordinal sequence (Scarf, Hayne, \& Colombo, 2011; Scarf, Danly, Morgan, Colombo \& Terrace, 2011; Scarf \& Colombo 2008). Using direct comparison within the same experimental group, subjects rely on the same numerical system for food and inherently non-valuable tokens (Addessi, Crescimbene, \& Visalberghi, 2008; Carey, 1998; Feigenson, Carey, \& Hauser, 2002).

Object representation. With respect to object representations, experiments on tracking and directed reaching reveal that human infants and primates reason about the motion of animate and inanimate kinds by way of two signature constraints: principle of cohesion (objects are connected bodies that maintain their connectedness as they move) and principle of continuity (objects move only on at least one connected path) (Hood, Hauser, Anderson, \& Santos, 1999; Spelke et al., 1992). Object representation is governed by a set of signature spatio-temporal principles inapplicable to agents. First, principles of continuity dictates that objects will move on unobstructed connected paths (Aguiar \& Baillargeon, 1999). Second, principles of cohesion determines that objects will move as bounded whole (Leslie \& Keeble, 1987; Valenza, Leo, Gava, \& Simion, 2006). Third, principles of physical contact observe that objects do not interact at a distance (Spelke, 1990). These features enable humans and animals to predict when and where objects will move or come to rest, perceive the boundaries of objects within a field of view, and conceive shape representations for objects that move out of view (Regolin, Vallortigara, \& Zanforlin, 1995; Santos, 2004; Valenza et al., 2006).

Objects in natural settings (human or nonhuman) are seldom found standing in isolation from one another, in continuous full view, or against a homogeneous background. For that reason, perception and representation are of objects in shifting, cluttered arrays rather than in isolated, static or homogeneous environments is particularly relevant to fully understanding an object representation system (Spelke, 1990). The ability to perceive objects in a rich diverse and changing environment requires processing features beyond those of surface layout to capture the 
underlying arrangement of structures that compose geometrical layout (Spelke, 1990), which ties directly into spatial representation abilities. Despite evolutionary divergence and differences in neural architecture, avian visual perception and complexity appears to show both mechanistic and behavioural similarities (Cook, 2000), such as the ability to generalise visual features when categorising (von Fersen \& Delius, 1989), and construct a generalised concept of a location or object based on multiple differing viewpoints (Cook, 2000; Honig \& Stewart, 1988). Behavioural evidence suggests that some avian visual acuity may exceed that of humans in certain contexts, for example, pigeons show no lag in reaction time for visual searches of reversal of features (Allan \& Blough, 1989), and no increased reaction time judging object identity when presented at different angles of orientation (Hollard \& Delius, 1982), suggesting a greater capacity to mentally rotate objects and visual information than primates.

Agent representation. Research on agency representation in CK reveals two consistent processing signatures with respect to making inferences from socialperceptual cues. In cooperative object choice paradigms, where subjects have to spontaneously read and use eye gaze to find the location of a hidden food, human infants pass while primates and corvids fail (e.g., Schloegl, Kotrschal, \& Bugnyar, 2007). In competitive foraging paradigms, however, primates and corvids "steal" more food from a conspecific or heterospecific competitor who is glancing away or whose eyes are closed than from one who is visually aware (e.g., Carter, Lyons, Cole, \& Goldsmith, 2008). Agency in terms of corvid cache protection strategy (Bugnyar \& Heinrich, 2006; Emery, Dally, \& Clayton, 2004) and anticipation of conspecific reaction (Emery \& Clayton, 2008) takes place within a competitive setting.

Distinguishing signature characteristics of agent representation are exactly those which do not define objects: goal-directedness, efficiency, contingency, reciprocity, and gaze direction (all of which are defining principles for nonhuman animals and humans alike) (Spelke \& Kinzler, 2007). According to Woodward(1999), agency involves actions directed to outcomes (goals), and agents achieve their goals through means that are efficient and rational (Gergely \& Csibra, 2003). Agents also interact contingently (Johnson, Booth, \& O'Hearn, 2001; Watson, 1972) and reciprocally (Meltzoff \& Moore, 1977). Newly hatched chicks, rhesus monkeys, and 
chimpanzees are sensitive to what their predators or competitors can and cannot see (Agrillo, Regolin, \& Vallortigara, 2004; Flombaum \& Santos, 2005; Hare, Call, \& Tomasello, 2001) Studies such as these are consistent with the physiological signatures of 'mirror neurons', which have been observed in captive monkeys and selectively respond to specific actions performed both by the self and others (Rizzolatti, Fogassi, \& Gallese, 2008).

Spatial representation. The overarching signature characteristic of spatial representation in humans and nonhuman animals (e.g. rats, desert ants, birds, etc.) alike is the ability to navigate or orient either themselves, or the location of an object within an area, based on geometric information in the environment, using shape as an indicator (Hermer \& Spelke, 1994). Non-geometric features do not appear to be universal in their utilisation; whereas adult humans take into account non-geometric information (e.g., surface colour), young children, rats and other species do not, even when that information is represented (Cheng, 1986; Hermer \& Spelke, 1996).

A more flexible spatial reorientation on the basis of surface cues (e.g., colour) or integrating geocentric and non-geometric cues may draw specifically on the unique compositionality of natural human language (e.g., Cheng, 1986; Gallistel, 1990; Spelke, Gentner, \& Goldin-Meadow, 2003). In the absence of language, however, arboreal monkeys living in changing rainforest environments do integrate metric relations between spatial elements with landmark information under certain conditions (e.g., Townsend, Clark, McGowan, \& Lovette, 2009). Orientation and navigation in avians appear to rely on both geometric and non-geometric information, enabling them to orient to angles of a location they have not yet been exposed to (Honig \& Stewart, 1988), and orientate and locate items in an array (Chiesa, Speranza, Tommasi, \& Vallortigara, 2006). A geometric CK domain may turn out to privilege only geometric cues or both geometric and non-geometric cue information depending on phylogenetic differences, experimental or developmental factors. Research over instinctive agent representations in human and animals is expansive given that reasoning about mental states involves many component skills (Call \& Tomasello, 1999; Krachun, Carpenter, Call, \& Tomasello, 2009; Low, 2010).

In recent years, the cognitive milestones that shape our understanding of uniquely human capacities have shifted dramatically as new evidence is found that continually redefines our perception of both universal and specialised cognitive 
function. Finding core areas of knowledge that function cognitively in a unilateral way across species with as distant as birds and mammals, speaks to the evolutionary significance of just how early these fundamental building blocks may have arisen.

\section{Cognition in the Wild}

Two distinct traits are common across the set of four CK domains of number, object, agency and spatial geometry which comprises the neural and cognitive mechanisms that form the basis of more complex psychological skills: First, they are common to infants, children and adults, and emerge early in human development. Second, they evolved before humans and so are shared with other species (Hauser, Spelke, \& Gazzaniga, 2004).

Animal cognition research uncovering evidence supporting CK 'signature' limits is fundamentally underpinned by research with primates (Boysen \& Berntson, 1989; Feigenson \& Carey, 2005; Santos, 2004; Spelke, 1990). There is a range of supporting studies on number representation in rats, fish, pigeons and, more recently, invertebrates (e.g., Agrillo, Dadda, \& Bisazza, 2007; Carazo, Font, FortezaBehrendt, \& Desfilis, 2009; Honig \& Stewart, 1989; Uller, Jaeger, Guidry, \& Martin, 2003; von Fersen \& Güntürkün, 1990).

Other forms of CK - reasoning about object physics and agent mental life - have yet to be comprehensively examined in a non-primate context. Fewer studies on CK in non-mammals address ethological relevance, evolutionary or species-specific implications. Evidence of parallel cognitive complexity in some avian species (e.g., brood-parasitic cowbirds, scrub jays, crows) point to birds as being prime candidates for examining ultimate causes (e.g., the adaptive value of cognitive and social reasoning) and proximate mechanisms (e.g., whether and how CK competencies change with age and map onto neurobiological structures) that result in behaviour production (e.g., Clayton, 1995; Low, Burns, \& Hauber, 2009). Demands on food caching in corvids, for example, necessitate complex observational and episodic-like memory capacities compared to birds that do not face the same pressures for storing and tracking food (e.g., Bednekoff, Kamil, \& Balda, 1997; Clayton, Emery, \& Dickinson, 2006a). The adaptive pressures surrounding food caching and pilfering may give rise to CK abilities in certain avian brains (Hunt, Low, \& Burns, 2008; Low et al., 2009). 
Cognition in wild animals is by its nature a difficult field of research. The vast majority of species on the planet are unsuited for an experimental study examining cognition simply because engaging the animal is extremely challenging - particularly when the human experimenter is naturally a predator or prey of a given species. Making observations at close range can also present a problem; many of the studies of species done in their natural habitat must sometimes done at a considerable distance (Marzluff, Walls, Cornell, Withey, \& Craig, 2010; McComb et al., 1994).

\section{New Zealand Robins}

The subjects in this study were wild North Island robins (see Figure 1); an endemic passerine whose populations are largely limited to protected forest areas (Armstrong \& Ewen, 2002; Armstrong et al., 2002; Armstrong, Raeburn, Lewis, \& Ravine, 2006a, 2006b). Robins live in mated pairs, defending a given territory within which they hunt primarily for invertebrates, cache food, and raise young (Powlesland, 1980). They are a food hoarding species that cooperate to provision young during the summer nesting season, and display conflict and competition for food resources with varying rates of aggression year-round (Steer \& Burns, 2008). Pilfering, relocating and redistributing both pilfered and self-hunted prey is present in both males and females; however, differing rates of retrieval have been observed between the sexes (Burns \& Van Horik, 2007).

The population of robins tested in this study is found within a native wildlife sanctuary forest (Zealandia) in Wellington, New Zealand. This sanctuary is controlled for introduced predators (such as cats, rats and stoats) using predator-proof fencing and baited traps, to protect native bird populations within the sanctuary from predation. Approximately 225 hectares in area, Zealandia is covered primarily in native broadleaf forest and encompasses a deep valley ranges from $140 \mathrm{~m}$ to $358 \mathrm{~m}$ in elevation. As of 2008 , it housed a population of approximately 150 colour-banded birds, and a total population estimated to just below 600 birds (McGavin, 2009). Robins, like other endemic birds of New Zealand, have evolved in the absence of terrestrial mammals and are particularly susceptible to predation due to naïve behaviour seen many isolated island populations (Menzies \& Burns, 2010). This naïve response to terrestrial mammals, in combination with food-hoarding and 
pilfering behaviour, makes an ideal wild candidate for comparative cognition research eliciting spontaneous responses.

New Zealand robins are one of a very small number of food-hoarding birds in the Southern Hemisphere (Vander Wall, 1990). Robins are monogamous and mated pairs reside on exclusive territories year-round (Burns \& Steer, 2006), as seen on the map of territories in Figure 2. This species is a medium-sized insectivorous passerine that is endemic to New Zealand, and found both on the North and South Island. Like many other animals native to isolated oceanic islands, robins lack pronounced anti-predatory behaviours and are fearless of humans. They will consume and cache foods offered to them by hand, and readily attend to and interact in experimental paradigms without extensive familiarisation or training (Hunt et al., 2008). They show complex cognitive abilities in creating, protecting and retrieving caches. Robins cache food in numerous different sites, regularly caching more than one item in each site. Further, when robins retrieve their own or pilfer their mates' caches, they fly to the exact location of these cache sites, even after leaving them for significant periods of time - suggesting accurate observational spatial memory (see Menzies \& Burns, 2008, for a review). Examining numerosity discrimination in a scatter-hoarding songbird may uncover what roles phylogeny, ontogeny and ecology play in shaping universal and species-specific features in the cognitive mechanisms underlying quantity representation.

The Petroicidae family of Australo-Papuan robins has a relatively small handful of members inhabiting New Zealand, including the New Zealand robin (Petroica australis), the tomtit (Petroica macrocephala), and the Chatham Island black robin (Petroica traversi) among others. While the North Island robin was previously classified as a subspecies of the subspecies of the New Zealand robin using the nomenclature Petroica australis longipes (Fleming, 1950), more recent evidence based on mitochondrial DNA suggests that North Island robins are in fact a distinct Petroica subspecies (Holdaway, Worthy, \& Tennyson, 2001; Miller \& Lambert, 2006), more accurately called Petroica longipes. For the purposes of these studies, which focus entirely on behavioural aspects of cognition, the minimal ecological and geographical differences between New Zealand and North Island robins are a distinction that is functionally irrelevant to the present context. This is especially true as important and salient behavioural features exist in both species, such as caching, 
pilfering, defence of stable territories, and pair bonding. Future studies looking at possible variations in behavioural or cognitive features among sub-groups that differ geographically, and genetically, would certainly add interesting and valuable ecological context to this species contribution in the growing body of literature that informs ecology, cognition and evolution as a whole.

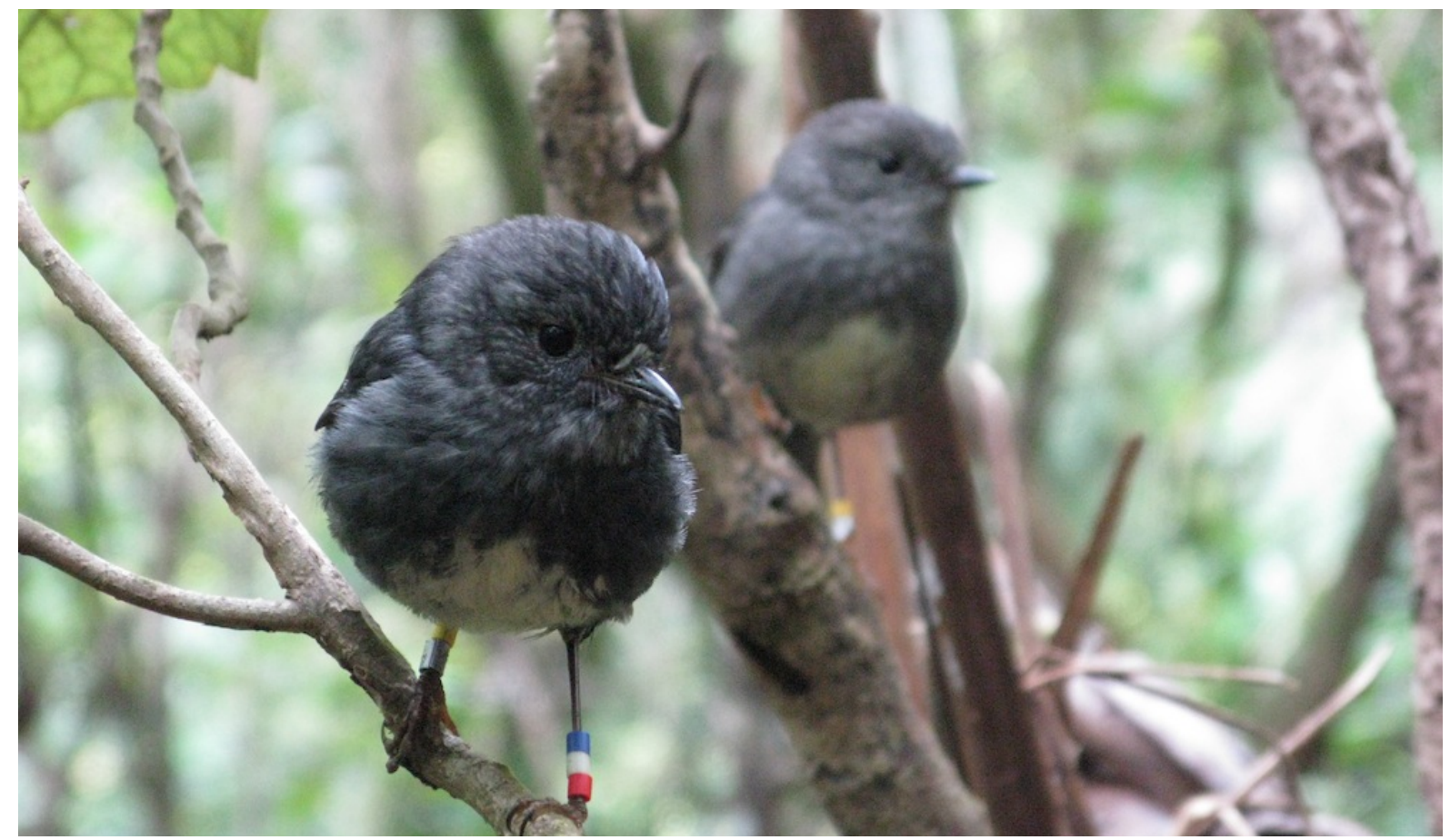

Figure 1: An adult mated pair of North Island robins (Petroica longipes)

The New Zealand robin is in many ways ideal as a model system for cognitive mechanisms in a naturalistic setting, using behavioural experimentation in the wild. As a species, the biological naiveté displayed is not as linear as one might think. The mainland robin population that remained (such as it was) did display responses to predators on mainland areas of the North Island to some extent (Maloney \& McLean, 1995), but have been found to quickly unlearn such behaviour in predator-free environments, showing almost no response within a single generation of relocation (Whitwell et al., 2011). Successful reintroduction of robins (along with other species) into off-shore islands and mainland reserves has seen largely successful breeding and re-population of these areas (Hanus \& Call, 2007; Taylor, Jamieson, \& Armstrong, 2005; Armstrong et al., 2002) but little to no behavioural defences (Whitwell et al., 2011). 


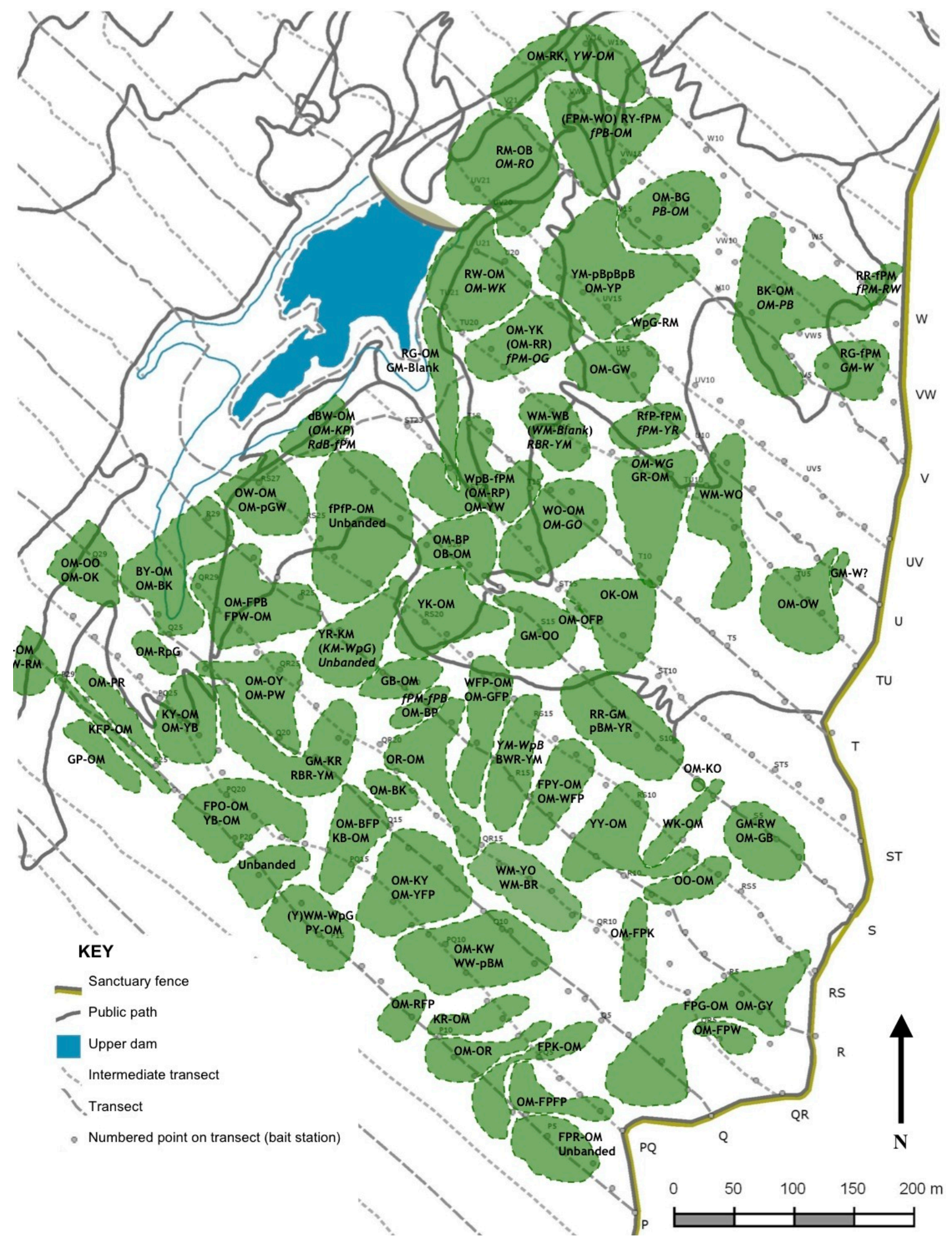

Figure 2: A map of robin territories in one portion of the sanctuary. 


\section{Research Questions}

A total of 43 individual robins took part in the studies described here (see Appendix $A$ ), ranging in approximate age at testing from less than 1 to 11 years. Individuals inhabited a range of the Zealandia Sanctuary along the eastern edge of the fenced forest reserve, close to the sanctuary entrance on the north end. Transect lines with markers and bait-stations were used in identification and documentation of robin territories and nesting sites (see Figure 2).

The objective of the suite of studies presented here is to establish whether the different core knowledge domains are similarly observed in the New Zealand robin and, further, whether there may be differences in the defining cognitive signatures in avian CK that reveal the species' divergent evolutionary history and unique ecological niche. The primary research questions are addressed by six studies, focused on two of the four core cognitive areas: Numerosity and Agency.

The first three studies address various features of numerosity in robins, asking several questions regarding robins' abilities to discriminate and compute varying quantities. How accurately do robins distinguish small and large quantities of prey items, and either ratio or numerical distance factors? Is the quantity discrimination ability of robins influenced or altered by spatially distributing the locations of two different quantities across multiple hidden arrays? Are robins able to successfully perform simple mathematical computations by responding differently to addition and subtraction of prey that is consistent or inconsistent with what they see?

The following three studies address several aspects of agency representation, and explore a number of questions about robins' capacity for responding to visible social cues in an evolutionarily distant and invasive species - humans. Can robins make pilfering decisions by taking into account the gaze of heterospecifics? Do robins make pilfering decisions by taking into account the visibility of limbs in heterospecifics? Do robins respond to cache retrieval and pilferage of live, paralysed or dead prey of various kinds differently depending on the species, mobility and animacy of the prey? 
CHAPTER 2: Study 1 - Discrimination of Large and Small Quantities²

${ }^{2}$ Contents from this chapter have been published as "Large quantity discrimination by North Island robins (Petroica longipes)" in Animal Cognition (2012) by Garland, Low \& Burns 
Quantity discrimination is fundamental to numerical cognition and considered an ability that is at the root of symbolic counting (Carey \& Williams, 2001; Gallistel \& Gelman, 1992). Not surprisingly then, studies examining relative quantity judgments (RQJs) have been conducted with human infants and adults (e.g., Barth, Kanwisher, \& Spelke, 2003; Feigenson et al., 2002) and primates (e.g., Beran \& Beran, 2004; Hanus \& Call, 2007; Wynn, Cummins, \& Allen, 1998) as well as other species ranging from insects to elephants (e.g., Agrillo, Dadda, Serena, \& Bisazza, 2008; Carazo et al., 2009; Irie-Sugimoto, Kobayashi, Sato, \& Hasegawa, 2009; Meck \& Church, 1983).

Non-linguistic number sense is understood to exist in humans and animals in two distinct systems; an indexing system, which processes or stores "object files" with a strict capacity limit of three to four items, and an analog magnitude system used in approximating larger numbers (Trick \& Pylyshyn, 1994; Uller, Hauser, \& Carey, 2001). Weber's law appears to play a fundamental role in the underlying mechanism with which the perception of large numbers is approximated (Cantlon, Platt, \& Brannon, 2009; Gallistel, Gelman, Holyoak, \& Morrison, 2005). According to this law, the size of the smallest perceptible difference in stimulus intensity varies as a function of a proportion of the original magnitude of that stimulus (Jordan \& Brannon, 2006). Within the framework of an analog magnitude system, ratio is the primary predictor of accuracy in discriminating between different quantities. There is still debate over whether there are two distinct core number systems. Some studies with monkeys and human infants support different cognitive signatures governing small versus large numbers (Feigenson, Dehaene, \& Spelke, 2004) whilst other studies have found that a singular mechanism based on Weber's law accounts for RQJs across a wide range of numerosities (e.g., Beran, 2001; Cantlon \& Brannon, 2006).

Despite increasing comparative research interest in quantity discrimination, understanding the ecological factors that determine when and how mental representations of quantity are deployed remains a challenge that needs to be addressed (Dehaene, Izard, Spelke, \& Pica, 2008; Emery \& Clayton, 2004; Feigenson et al., 2002). The goal here was to investigate spontaneous quantitative cognition skills in wild New Zealand robins, specifically North Island robins (Petroica longipes), an endemic species of New Zealand (Hunt et al., 2008). The bulk of the data on spontaneous numerical discrimination is still primate and mammal centric 
(Carazo et al., 2009; Dadda, Piffer, Agrillo, \& Bisazza, 2009; Uller et al., 2003). Considering the evolutionary distance of birds and mammals, examining RQJs in a scatter-hoarding songbird will contribute to a more phylogenetically diverse picture of numerical cognition (Beran, 2008; Clayton, 1995). There is work on numerical abilities in birds, but they involve considerable amounts of training and/or relate primarily to discrimination of sets with small sizes (Farnsworth \& Smolinski, 2006; Honig \& Stewart, 1989; Koehler, 1941; Pepperberg, 2006; Rugani, Regolin, \& Vallortigara, 2008; Terrace, 1987). However convincingly studies demonstrate animal learning and cognition after extensive training, interpreting what the findings mean in terms of animals' natural ability to represent number remains difficult (Davis \& Pérusse, 1988; McComb et al., 1994).

There are some studies that hint at wild birds' adaptive number sense. For example, American coots experience nest parasitism from conspecific females, and egg number combined with recognition contribute to clutch size decisions. Lyon (2003) found that female coots that discriminated parasitic eggs from their own counted only their own eggs and thus avoided inadvertent clutch size reductions. Quantity discrimination has also been suggested to partly underpin Brown-headed cowbirds' (Molothrus ater, an obligate brood parasite) synchronisation of nest parasitism with host incubation readiness. White, Ho, and Freed-Brown (2009) found that cowbirds spent longer periods investigating nests with the correct accumulated number of eggs in relation to elapsed days, suggesting that cowbirds appear to remember changes in egg-number between visits. The few studies on number cognition in wild birds have, however, focused on only a narrow range of numerical values and with small set sizes (Farnsworth \& Smolinski, 2006; Low et al., 2009). Some of these sets are still relatively 'large' in terms of being above object-file threshold of 3 to 4 items, but not substantially large enough to establish whether there is a sophisticated upper limit to birds' number discrimination or what representational system could underpin such capacities. This is important to address when assessing numerosity in birds where certain food-storing species have been shown to display significant spatial attention and memory skills (Clayton, Dally, Gilbert, \& Dickinson, 2005; Hunt et al., 2008).

North Island robins, the species of the current study, are one of a very small number of food-hoarding birds in the Southern Hemisphere (Vander Wall, 1990). 
Robins are monogamous and mated pairs reside on exclusive territories year-round (Burns \& Steer, 2006). This species is a medium-sized insectivorous passerine that is endemic to New Zealand, and found both on the North and South Island. Like many other animals native to isolated oceanic islands, robins lack pronounced antipredatory behaviours and are fearless of humans. They will consume and cache foods offered to them by hand, and readily attend to and interact in experimental paradigms without extensive familiarisation or training (Hunt et al., 2008). They show complex cognitive abilities in creating, protecting and retrieving caches. Robins cache food in numerous different sites, regularly caching more than one item in each site. Further, when robins retrieve their own or pilfer their mates' caches, they fly to the exact location of these cache sites, even after leaving them for significant periods of time - suggesting accurate observational spatial memory (see Menzies \& Burns, 2008, for a review). Examining numerosity discrimination in a scatter-hoarding songbird may uncover what roles phylogeny, ontogeny and ecology play in shaping universal and species-specific features in the cognitive mechanisms underlying quantity representation.

Initial RQJ testing by Hunt et al. (2008) indicated that robins chose the cache site with greater number more than half the time with comparisons involving 1 vs. 2,2 vs. 3,3 vs. 4,4 vs. 5 , and 4 vs. 8 , but chose at chance or below with 4 vs. 6 ; and 6 vs. 8 and 8 vs. 10. Their analysis indicated that total set size rather than ratio predicted the accuracy of robins' numerical judgments. Precise numerical judgments could potentially form an integral part of robins' cache retrieval strategy. If robins are able to compute precisely how many pieces of prey items are in each of its (and its mates') cache sites, it would help prioritise efficient cache retrieval and pilfering (Burns \& Steer, 2006). Moreover, given that robins only store insect prey that are highly perishable, tracking and remembering the precise number of items stored in particular cache sites would help prioritise cache retrieval to minimise losses to spoilage. Hunt et al.'s findings are illuminating in their contrast with work on primates and human infants showing that the object-file system, whilst capturing performance with small sets, cannot process quantities greater than 3 or 4 items simultaneously. The results suggest that robins - in the context of food-storing and food-retrieval demands - may have developed an object indexing system with a higher upper limit. 
In relation to the present study, though, it is still not clear whether robins have relatively sophisticated discrimination of numbers in an absolute sense, or whether a single fuzzy analog magnitude system for recognising all quantities - large or small underpins RQJs. First, in Hunt et al.'s (2008) study, all comparisons with ratios at 0.50 , half at 0.67 and 0.75 and none at 0.80 were significant. This suggests that ratio, to some extent, may influence robins' numerosity discrimination, but it was undetected due to the limited range of comparisons being examined. Second, Hunt et al. tested RQJs by presenting the two sets of quantities item-by-item whereby the totality of items was never visually available at the time of cache selection, as prey was dropped into opaque containers and subsequently covered. Research indicates that apes (Hanus \& Call, 2007) and South American sea lions (Abramson, Hernández-Lloreda, Call, \& Colmenares, 2011) are poorer at spontaneously selecting the larger value when the two sets of quantities were presented item-byitem compared to when the two quantities are presented simultaneously as visually accessible whole sets. Importantly, ratio between quantities was found to account for apes and sea lions' spontaneous RQJ performance - analog magnitude representations appear to be especially favoured when items are visibly presented as a united set (see Shettleworth, 2009).

There is compelling evidence in some studies (Beran, 2001, 2004) that chimpanzees are able to make highly accurate RQJs (70-95\% correct) even when larger numbers of food items are sequentially presented and hidden from view (5 vs. 8,5 vs. 10 , and 6 vs. 10). However, as Beran notes (2004), it is not clear whether certain subjects' rich history of participation in numerical and other cognitive tasks may have scaffolded their ability to update and retain quantity information when sets are sequentially presented. Overall, while each of these methods (quantities presented simultaneously as whole sets or quantities presented item-by-item) has been frequently used to test animals' RQJs, there are few systematic comparisons of spontaneous performance between these two types of tasks (Abramson et al., 2011; Hanus \& Call, 2007). It is noteworthy that wild robins in Hunt et al.'s study could make RQJs successfully in a cognitively demanding task context where the item-byitem presentation meant that the birds had to deal with temporal discontinuity between prey items. Hunt et al. have suggested that an extended upper limit to robins' parallel individuation of food items may reflect these birds' sensory, motor, 
cognitive and neuroanatomical specialisations in behaviour for scatter-hoarding, pilfering and retrieving food. However, emphasising a sophisticated object indexing system operating in robins instead of a generalised and evolutionarily primitive Weber-based magnitude representation system may be premature.

Comparing robins' RQJs across different sets of quantities (high and low numbers weighted across the ratio scale) and in different task contexts (item-by-item and simultaneous presentation of visually accessible whole sets) can help uncover the commonality and diversity in the nature of the cognitive representations governing robins' quantitative judgments. The aim was to test whether robins converged with other species in having both object-file representations and analog magnitude representations, yet may diverge in terms of when limits in precise discrimination are imposed and when fuzzy Weber's law-based discrimination of quantities are deployed.

Three experiments were carried out to test robins' spontaneous RQJs and investigate what representational systems could characterise their abilities. Experiment 1 tested robins' RQJ using an item-by-item paradigm where 10 different number pair comparisons (from 1 vs. 8 to 14 vs. 16) were combined with 5 different ratios (from 0.125 to 0.875 ), so that the total number of worms remained hidden from view as each robin made its choice. Experiment 2 presented the same number comparisons (and ratio range) to a new group of robins, by pouring the two sets of worms simultaneously, so two quantities remained visually accessible at the time of choice. In Experiment 3 another new group of robins were tested on RQJs with the same visually accessible context in Experiment 2, using number comparisons 4 to 8 times larger than those presented in the first two experiments (see Table 1). An analysis across all three experiments was conducted, examining the effects of ratio between pairs of quantities and total set size across pairs of quantities to contrast the analog magnitude and object indexing systems. 
Table 1. Number comparisons and total number of worms displayed for each trial for the 5 ratios investigated across Experiments 1,2 and 3.

\begin{tabular}{|c|c|c|c|c|}
\hline \multirow[t]{2}{*}{ Ratio } & \multicolumn{2}{|c|}{ Experiments $1 \& 2$} & \multicolumn{2}{|c|}{ Experiment 3} \\
\hline & Comparison & Total & Comparison & Total \\
\hline \multirow[b]{2}{*}{0.125} & 1 vs. 8 & 9 & 4 vs. 32 & 36 \\
\hline & 2 vs. 16 & 18 & 8 vs. 64 & 72 \\
\hline \multirow{2}{*}{0.250} & 1 vs. 4 & 5 & 8 vs. 32 & 40 \\
\hline & 2 vs. 8 & 10 & 16 vs. 64 & 80 \\
\hline \multirow{2}{*}{0.500} & 4 vs. 8 & 12 & 16 vs. 32 & 48 \\
\hline & 8 vs. 16 & 24 & 32 vs. 64 & 96 \\
\hline \multirow{2}{*}{0.750} & 3 vs. 4 & 7 & 24 vs. 32 & 56 \\
\hline & 6 vs. 8 & 14 & 48 vs. 64 & 112 \\
\hline \multirow[b]{2}{*}{0.875} & 7 vs. 8 & 15 & 28 vs. 32 & 60 \\
\hline & 14 vs. 16 & 30 & 56 vs. 64 & 120 \\
\hline
\end{tabular}

\section{Methods}

Subjects. Twelve individual robins participated in each experiment (36 robins in total). No subject participated in more than one of the three experiments described below. Each subject was identified with the unique combination of coloured bands on the bird's legs (see Figure 3). None of the robins used in this study had prior experimental history. This study was conducted in native New Zealand forest within the Karori Sanctuary. The sanctuary is located on the southern tip of the North Island of New Zealand ( $41^{\circ} 18^{\prime} \mathrm{S}, 174^{\circ} 44^{\prime} \mathrm{E}$ ) in Karori, Wellington. As of 2008 , it housed a population of approximately 150 colour-banded birds, and a total population estimated to be roughly 600 birds(McGavin, 2009). Robins used in trials were located auditorily and visually along a series of footpaths or transects traversing the Karori Sanctuary. 


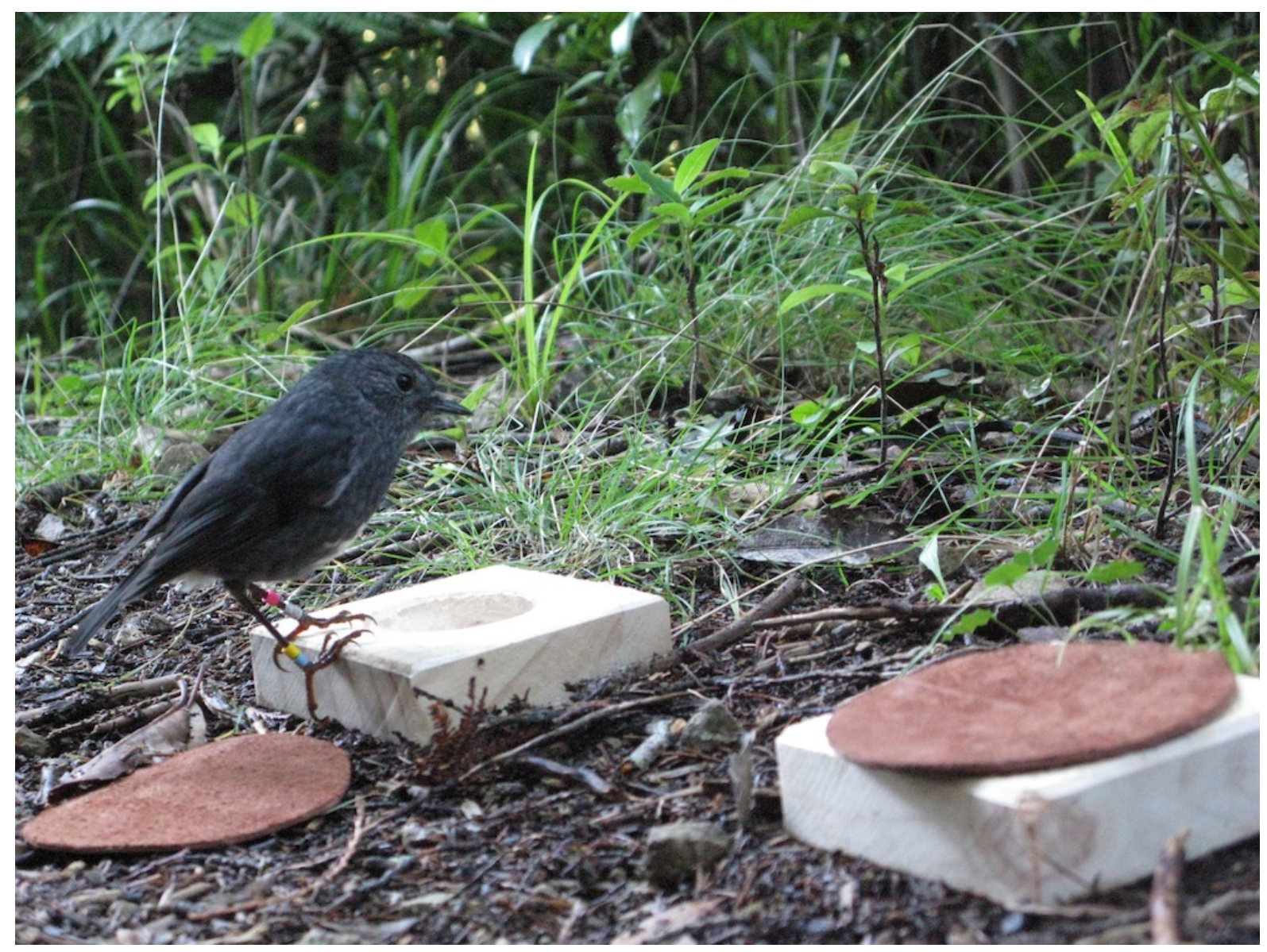

Figure 3. A robin makes his choice in a sequential number task.

Materials. This experiment examined RQJs by presenting subjects with mealworms (Tenebrio molitor larvae) in artificial caches comprising two plastic wells, sunk into a wooden encasement. The plastic lining of each well served to prevent the mealworms from being able to climb the rough wooden surface of the encasement, thus retaining even large quantities of mealworms within the encasement. Each well measured approximately $2 \mathrm{~cm}$ deep and they were spaced approximately $35 \mathrm{~cm}$ apart, allowing for variations in the terrain of the individual robin's territory. In Experiment 1, the wells were each covered with a circular brown leather flap approximately $7 \mathrm{~cm}$ across. For Experiments 2 and 3 , pre-counted containers of mealworms were poured into these wells from white plastic $35 \mathrm{~mm}$ film canisters. No covers were utilised for the wells in Experiments 2 and 3.

Procedure. Mealworm prey was presented to wild robins in an experimental arena containing two artificial cache sites (see Appendix $C$ for links to example videos). Wild robins frequently turn over leaves in search of prey on the forest floor. As a result, all birds readily removed leather lids to access prey without training after 
a brief exposure period to the experimental materials. For all three experiments, subjects were not positively or negatively reinforced for a specific choice, and allowed to retrieve mealworms for consumption or caching regardless of the choice made, but only from one of the two caches. The order of treatments was randomised in all three experiments in an attempt to control for observational learning.

In each experiment, 12 individual robins participated, for a total of 36 subjects across the three experiments, all of whom were colour-banded with unique identifying band combinations. Trials were conducted within the forest territory of each bird. Ten different number comparison spanning 5 ratios were displayed to each robin (see Table 1), with the total combined number of worms displayed ranging from between 5 and 120 across all three experiments. The number combinations in these three experiments (see Table 1) were specifically chosen for an even and broad distribution of ratios between 0 and 1 , and to create essentially 4 tiers of varying set sizes for each ratio, using 5 different larger set quantities $(4,8$, $16,32,64)$. There was no trial in which a choice was made and worms were not retrieved by the robin; every robin made a choice for the given set of 10 number comparisons. In all three experiments, presentation of the larger number on the right or left was counterbalanced and randomised, and the first number presented for Experiment 1 was also randomised for side (left, right) and quantity (more, less).

In item-by-item presentation, subjects are unable to see either the accumulation in each well as prey was added, or the final amounts (singly or simultaneously), as the opaque containers obscured prey, and were covered upon filling. Each individual mealworm is only visible as it is held in tweezers while being dropped into the well. It is important to note that the visible presentation procedure in Experiments 2 and 3 were specifically adapted for experimentation involving large quantities with a wild species, and prey were therefore not placed sequentially onto the platforms as in Experiment 1. In aiming to experiment with quantities of mealworms beyond a total number of 30 , it was important to reduce the time it took to place the worms onto the platform, because in many cases, robins will not attend to a trial for longer than about 60s. To this end, pouring the worms simultaneously in trials, for example, using a total of 80 or 120 worms, was essential to the participation of the subjects. To be able to appropriately compare both of these changes in methodology (visibility and manner of placement), Experiment 2 was also conducted using this pouring 
methodology and with the prey remaining visible, using the same smaller numbers as in Experiment 1.

Experiment 1. Hidden quantities of mealworms were presented item-by-item, numbering in combined total between 5 and 30. Ten specific number combinations consisting of five different ratios (see Table 1) were presented to all 12 subjects comprising 120 trials. Mealworms were placed in each site sequentially, at an approximate rate of $2 s$ per item, using metal tweezers, to prevent worms from being obscured as they were placed. Wells were separately filled with between 1 and 16 mealworms in each, and then covered. Due to the depth of the well, and the position of the subjects sitting low to the ground, robins did not have visual access to the accumulating contents of each well either during or after filling. The robin was then allowed to make a choice between the two artificial caches by removing the cover and retrieving the worms for consumption or storage.

Experiment 2. Each of the same ten number combinations and five ratios presented in Experiment 1 (Table 1) were simultaneously presented in whole sets to each of 12 different subjects. Subjects were presented with two caches, which were then simultaneously filled with between 1 to 16 mealworms each and remained visible. White plastic film canisters were pre-loaded with a specific number of mealworms. Mealworms were poured into the wells using the canisters.

Experiment 3. The final experiment presented very large numbers (up to 64 in a single set) to explore the upper range of robins' spontaneous numerosity discrimination ability. Ten new number combinations (Table 1) that spanned the same five ratios used in Experiments 1 and 2 were presented simultaneously as whole sets to each of 12 new birds, totalling 120 separate trials (10 per individual). Testing very large number discrimination in the wild with item-by-item presentation is not practical: ecological necessities faced by robins - vigilant monitoring of extant food caches and defending of territory from intruders - make it extremely unlikely that a given subject will attend to a sequential presentation of up to 120 worms. The experimental caches were simultaneously filled with between 4 and 64 mealworms in each well and remained visible. White plastic film canisters were pre-loaded with a specific number of mealworms for each number combination. Mealworms were poured into the wells using identical white film canisters. The robin was then allowed to make a choice between the two artificial caches, and the outcome documented. 


\section{Results}

Each of the three experiments examined the extent to which accuracy of RQJs varied with ratio and total set size, and additionally a joint analysis of Experiments 2 and 3 was performed across all visibly presented trials. Subjects' choice in each trial is defined as 'successful' where the greater of the two numbers was chosen. The ratio of the mealworms displayed was calculated dividing the smaller number (in one well) by the larger (in the second well) (e.g., 1/8 =.125). Total set size was the combined number of mealworms presented in both wells (e.g., 2 vs. $5=7$ ). Robins did not appear to display a side-bias across the three experiments in making a choice (left 51\%, right 49\%).

A binary logistic regression analysis for both ratio and set size was conducted for each of the 3 experiments, as well as a joint analysis of experiments 2 and 3, with each analysis including robin (individual subject) as a categorical variable to account for the within-subjects design. There was considerable variation between individuals, ranging from $40 \%$ by an individual in Experiment 3 to $100 \%$ correct by two individuals in Experiments 1 and 2. Robin, as a variable, was never a significant factor in the resulting model across trials presented in either Experiment $1(p=$ 0.863, Wald $=6.149, d f=11)$, Experiment $2,(p=0.985$, Wald $=3.383, d f=11)$ or Experiment $3(p=0.348$, Wald $=12.218, d f=11)$.

In a binary logistic regression, the odds ratio $(\operatorname{Exp}(B)$ refers to the decrease in the dependent variable (percentage of correct RQJs), which occurs with every unit of increase in the independent variable (e.g., total number of worms). So for example, when $(\operatorname{Exp}(B))=0.720$, the model indicates that with each unit increase - in this example, with every worm added to the overall number shown - the odds of a subject choosing correctly are reduced to a projected average of 0.72 times the odds of the previous comparison. This effectively reduces the odds of success in this example by $28 \%$ (or 0.28 times) with every added worm - decreasing the overall likelihood of success as set size gets larger.

Preliminary analyses indicated that there was no evidence of improvement in accuracy across trials in any of the three experiments (Exp. 1, $p=0.451$; Exp. 2, $p=$ 0.161 ; Exp. $3, p=0.657$ ), as shown in the results of a binary logistic regression. Individual robins were therefore not significantly more or less likely to be successful when responding on their tenth trial than their first; subjects' responses were 
spontaneous and non-reinforced as intended. Across all three experiments, few number comparisons were performed at chance (Experiment 2, 8 vs. 16 and 14 vs. 16; Experiment 3, 28 vs. 32 and 56 vs. 64 ) or below (Experiment 3, 48 vs. 64).

Experiment 1. Robins' RQJs for sequentially displayed comparisons involving trials with a total of 5 to 30 prey items are shown in Figure 4. Performances across the number combinations ranged from $58 \%$ success ( 14 vs. 16 ) to $100 \%$ success ( 1 vs. 4).

A binary logistic regression was conducted, including individual as a categorical variable, and prey ratio and total set size as independent covariates, with 'success' as the dependent variable. This revealed total set size to be a significant predictor of RQJ performance, $(p=0.043, \operatorname{Exp}(B)=0.936$ Wald $=4.096, d f=1)$, whereas ratio was not $(p=0.533, \operatorname{Exp}(B)=0.577$ Wald $=0.388, d f=1)$. The model indicated that with each unit increase in total set size for a number comparison, the odds were reduced to a projected average of 0.94 times the odds of the previous comparison, slightly decreasing (by $6 \%$ with each additional worm) the overall likelihood of success as total set size gets larger. Further analysis of Experiment 1 is conducted and explained as part of the following joint analysis section.

(a)

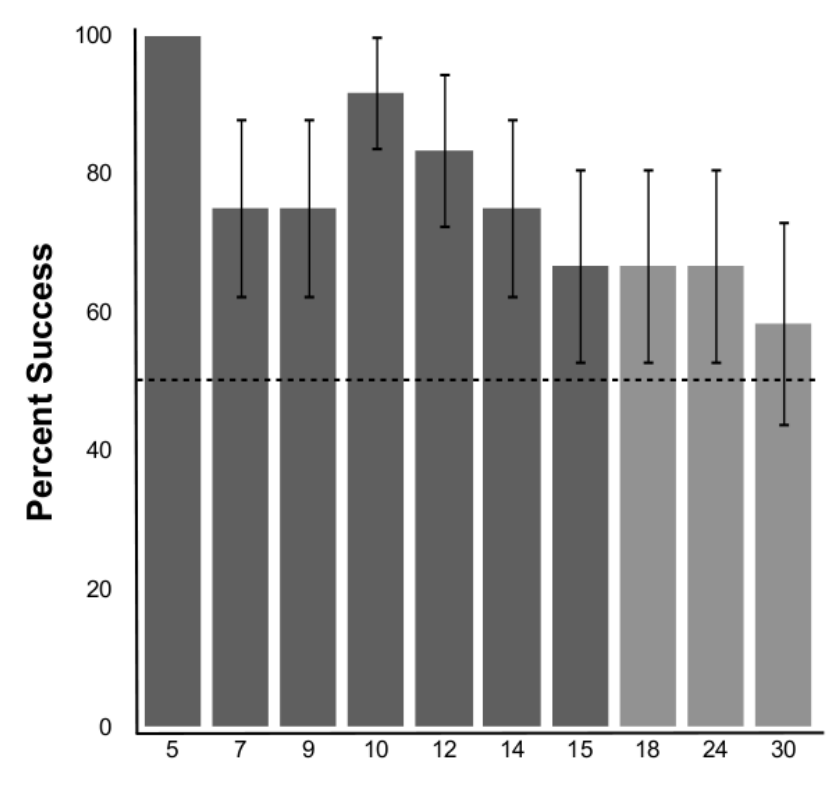

Total Number of Worms (b)

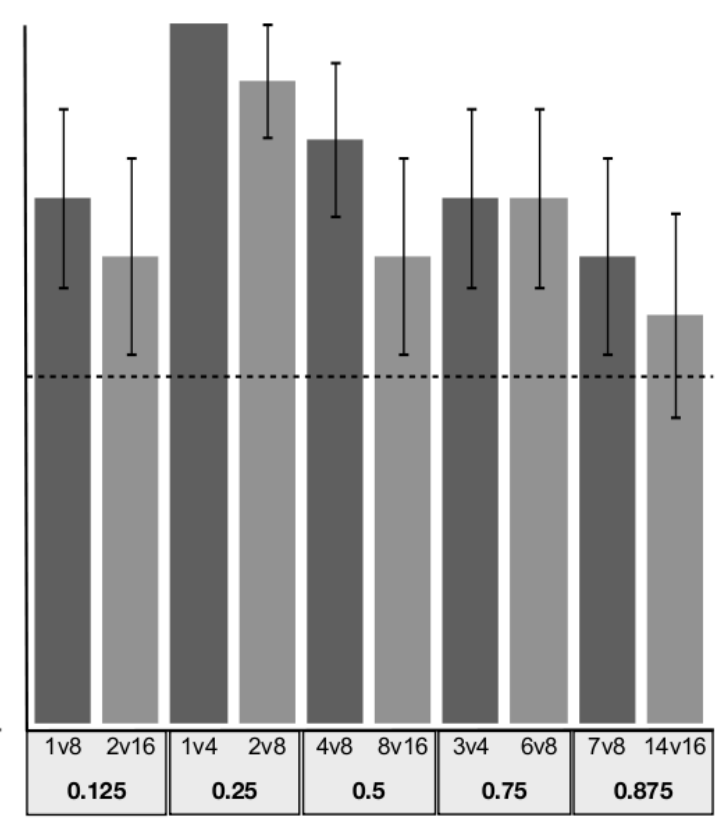

Ratio

Figure 4. Percentage of correct trials as a function of (a) ratio and (b) total number of worms (combined set size), for item-by-item presentation of comparisons containing up to 30 total prey items $(\mathrm{N}=12)$. 
Experiment 2. Robins' RQJs for simultaneously visible comparisons involving trials with a total of 5 to 30 prey items are shown in Figure 5 . Percent success overall ranged from between $50 \%$ ( 8 vs. 16 and 14 vs. 16 ) and $92 \%$ ( 1 vs. 8 ), showing a slight but non- significant $(t(22)=0.81, p=0.42)$ overall decrease in accuracy $(\mathrm{n}=$ $120, M=72 \%, S D=0.453, S E=0.041$ ) compared to the hidden trials of the same numbers in Experiment $1(n=120, M=76 \%, S D=0.430, S E=0.039$ ).

As in Experiment 1, the logistic regression analysing both prey ratio and total set size (with individual as a categorical variable), found total set size was significant ( $p$ $=0.034, \operatorname{Exp}(B)=0.936$ Wald $=4.484 d f=1)$. The model indicated that with each unit increase (worm added) in total set size, the odds were reduced to a projected average of 0.94 times that of the previous comparison, slightly decreasing (by $6 \%$ with each additional worm) the overall likelihood of success as total set size increases. Ratio was not a significant predictor of success $(p=0.394, \operatorname{Exp}(B)=$ 0.494 Wald $=0.727 d f=1$ ). While the model projected that the odds would be reduced to 0.49 times that of the previous comparison, drastically decreasing (by $51 \%$ with each additional worm) the overall likelihood of success as ratio increases, it remained a non-significant predictor of success.

(a)

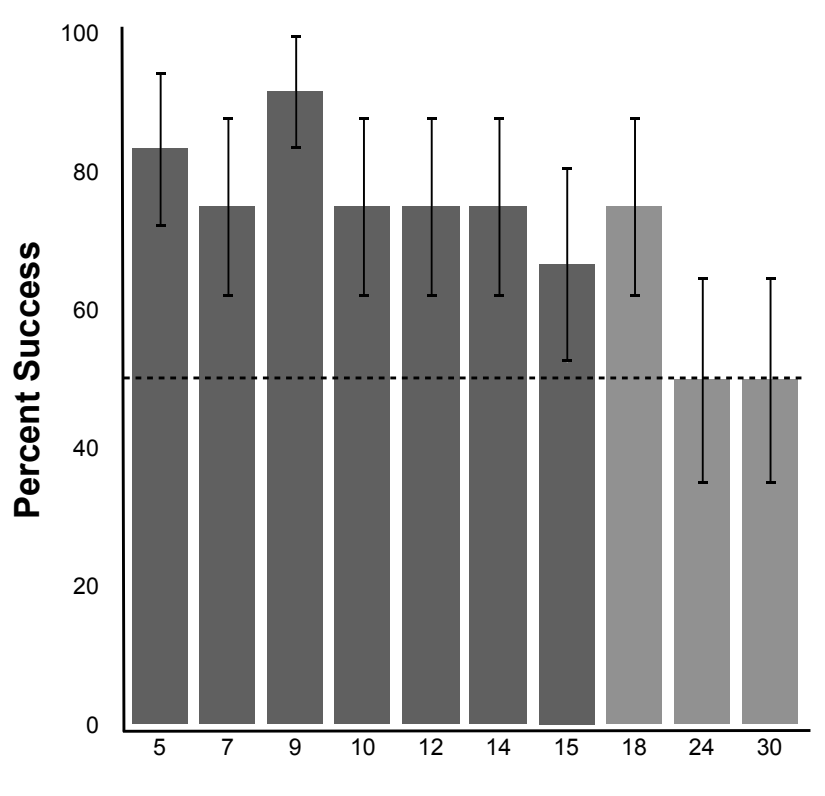

Total Number of Worms (b)

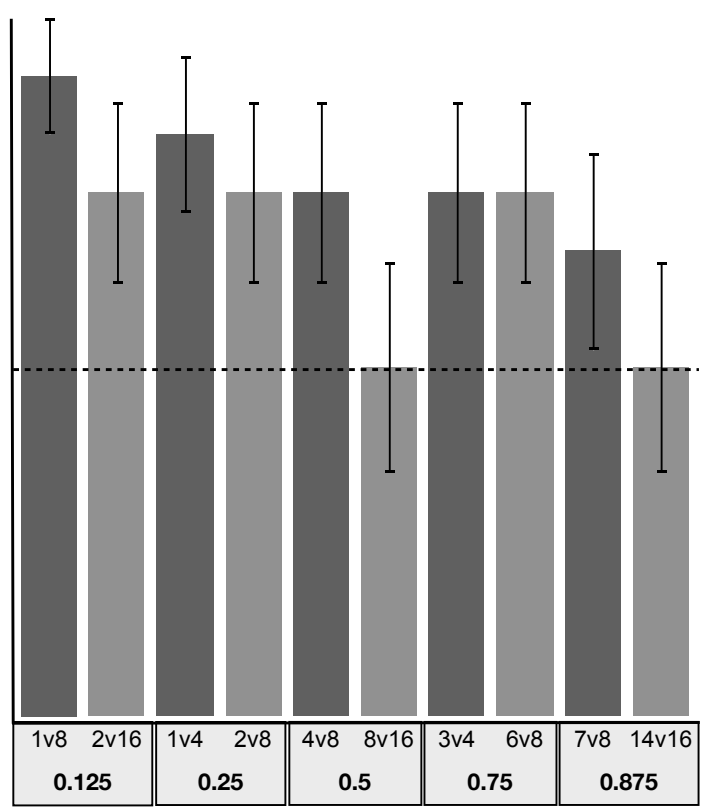

Ratio

Figure 5. Percentage of correct trials as a function of (a) ratio and (b) total number of worms (combined set size), for whole set presentation of comparisons containing up to 30 total prey items $(\mathrm{N}=12)$. 
Experiment 3. Robins' RQJs for simultaneous visible comparisons involving trials with a total of 36 to 120 prey items are shown in Figure 6 . Percent success overall ranged from between $33 \%$ (48 vs. 64 ) to $83 \%$ ( 4 vs. 32 and 8 vs. 64 ), on the whole lower $(n=120, M=63 \%)$ than Experiments $1(n=120, M=76 \%)$ or $2(n=120 M=$ $72 \%)$. The overall range of percentage of 'correct' decisions shows a slight decrease accuracy in contrast to both Experiment 1 (a significant difference, $t(22)=2.08, p=$ 0.049 ) and Experiment 2 (non-significant: $t(22)=1.44, p=0.17$ ), and response variance increased $(S D=0.484, S E=0.440)$ as ratio between sets increased.

A logistic regression including prey ratio and total set size (with individual subject as a categorical variable, and 'success' as the dependent variable), revealed that ratio was a strong significant predictor of success $(p=0.020, \operatorname{Exp}(B)=0.138$ Wald $=$ $5.412, d f=1)$, while total set size was not $(p=0.571, \operatorname{Exp}(B)=0.995$ Wald $=0.320$, $d f=1$ ). According to this model, the odds of the likelihood of making a successful choice is reduced to a projected average of 0.14 times that of the previous comparison, sharply decreasing (by $86 \%$ with each unit) the overall likelihood of success as prey ratio gets larger.

(a)

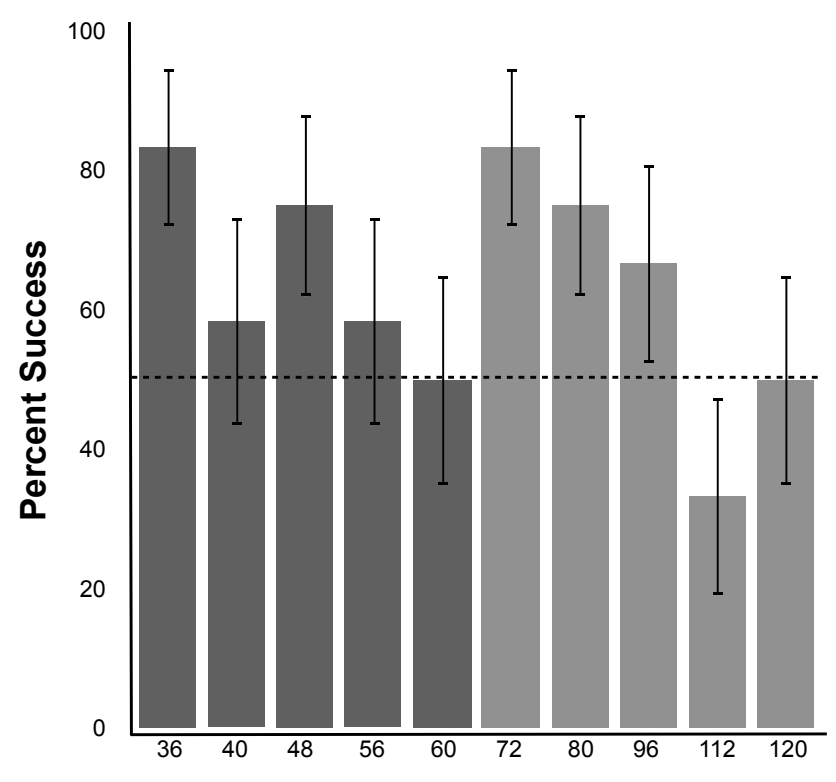

Total Number of Worms (b)

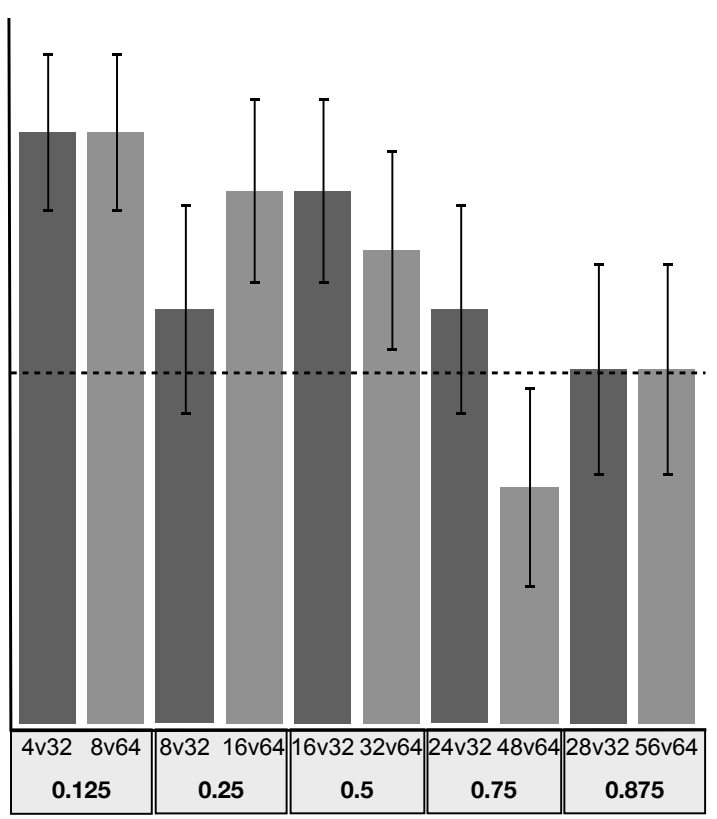

Ratio

Figure 6. Percentage of correct trials as a function of (a) ratio and (b) total number of worms (combined set size), for whole set presentation of comparisons containing up to 120 total prey items $(\mathrm{N}=12)$. 
Joint analyses. In a joint analysis of Experiments 2 and 3, both of which used the same methodology of visibly presenting varying quantities of prey, ratio was a significant predictor of success $(p=0.009, \operatorname{Exp}(B)=0.220$ Wald $=6.817, d f=1)$, while total set size was not $(p=0.206, \operatorname{Exp}(B)=0.990$ Wald $=1.603, d f=1)$. This overall result is expected, when for both high and low numbers, the model showed an extremely strong potential reduction in success associated with ratio both in Experiments 2 (by $51 \%$ per unit change) and 3 (by $86 \%$ per unit change), although it was only a significant predictor in the latter. With comparison, total set size remains a weak effect in the models for both Experiments 2 (accuracy reduced by 6\% per unit change) and 3 (a less than 1\% drop in accuracy per unit change), although the set size effect was only significant in the former.

Most importantly, when percent success is looked at across trials in both experiments (see Table 2), a pattern emerges indicating that in considering the larger of the two set sizes shown, success drops significantly $(t(18)=3.780, p=0.001$; see Figure 6) from the percent success of smaller sets, with a larger set size of 4 or 8 (n $=84, \mathrm{M}=77 \%, \mathrm{SD}=0.421)$, until the larger set size reaches $16(\mathrm{n}=36, \mathrm{M}=58 \%$, $S D=0.500)$. Beyond this, a split occurs where number comparisons with smaller numerical distance between the two sets (ratios of 0.750 and 0.875 ) remain close to chance, and those with greater numerical distance (ratios of $0.125,0.250$ and 0.500 ) increase again in the percentage of successful responses. This serves as an important marker for defining and analysing "small" and "large" quantities in reference to robins' performance with comparison to other species, and could be indicative of two systems at work.

In order to more accurately examine how ratio and total set size affect 'small' and 'large' numbers, experiments 1 and 2 have been reanalysed applying this new definition of 'large' and 'small' based on the findings of the joint analysis. To generate a data set of 'small' set sizes, all trials with a larger set size of 16 or more were removed, effectively reducing the definition of "small" set sizes to those where the larger set is either 4 or 8 in these experiments, again using a binary logistic regression. To generate a data set of 'large' set sizes, trials from Experiment 2 and Experiment 3 (both of which were presented in a simultaneous, visible manner) that had a larger set size of 16 were used. 
In Experiment 1, when trials with a larger set size of 16 or higher are removed from the model, neither ratio $(p=0.344, \operatorname{Exp}(B)=0.247$ Wald $=0.895, d f=1)$ nor total set size $(p=0.393, \operatorname{Exp}(B)=0.903$ Wald $=0.730, d f=1)$ are significant predictors of success. Likewise, in Experiment 2, when trials with a larger set size of 16 or higher are removed from the model, neither ratio $(p=0.344, \operatorname{Exp}(B)=0.247$ Wald $=0.895, d f=1)$ nor total set size $(p=0.393, \operatorname{Exp}(B)=0.903$ Wald $=0.730, d f=$ 1) are significant predictors of success. In Experiment 3, when trials from Experiment 2 with a larger set size of 16 are included in the model, again, ratio emerges as a strong significant predictor of success, and total set size is not significant. These results indicate that set sizes below 16 square with a more object-based paradigm, but do not fall in line with the strict 4-item limit imposed by a typical "object-file system", while numbers 16 and above are subject to a ratio-based system.

Table 2. Percentage of correct trials across Experiments 1, 2 and 3 in both 'small' and 'large' comparisons.

\begin{tabular}{|c|c|c|c|c|c|c|}
\hline \multirow[t]{2}{*}{ Ratio } & \multicolumn{2}{|c|}{ Experiment 1} & \multicolumn{2}{|c|}{ Experiment 2} & \multicolumn{2}{|c|}{ Experiment 3} \\
\hline & Small & Large & Small & Large & Small & Large \\
\hline \multirow{2}{*}{0.125} & 1 vs. 8 & 2 vs. 16 & 1 vs. 8 & 2 vs. 16 & 4 vs. 32 & 8 vs. 64 \\
\hline & $75 \%$ & $67 \%$ & $92 \%$ & $75 \%$ & $83 \%$ & $83 \%$ \\
\hline \multirow{2}{*}{0.250} & 1 vs. 4 & 2 vs. 8 & 1 vs. 4 & 2 vs. 8 & 8 vs. 32 & 16 vs. 64 \\
\hline & $100 \%$ & $92 \%$ & $83 \%$ & $75 \%$ & $58 \%$ & $75 \%$ \\
\hline \multirow{2}{*}{0.500} & 4 vs. 8 & 8 vs. 16 & 4 vs. 8 & 8 vs. 16 & 16 vs. 32 & 32 vs. 64 \\
\hline & $83 \%$ & $67 \%$ & $75 \%$ & $50 \%$ & $75 \%$ & $67 \%$ \\
\hline \multirow{2}{*}{0.750} & 3 vs. 4 & 6 vs. 8 & 3 vs. 4 & 6 vs. 8 & 24 vs. 32 & 48 vs. 64 \\
\hline & $75 \%$ & $75 \%$ & $75 \%$ & $75 \%$ & $58 \%$ & $33 \%$ \\
\hline \multirow{2}{*}{0.875} & 7 vs. 8 & 14 vs. 16 & 7 vs. 8 & 14 vs. 16 & 28 vs. 32 & 56 vs. 64 \\
\hline & $67 \%$ & $58 \%$ & $67 \%$ & $50 \%$ & $50 \%$ & $50 \%$ \\
\hline
\end{tabular}




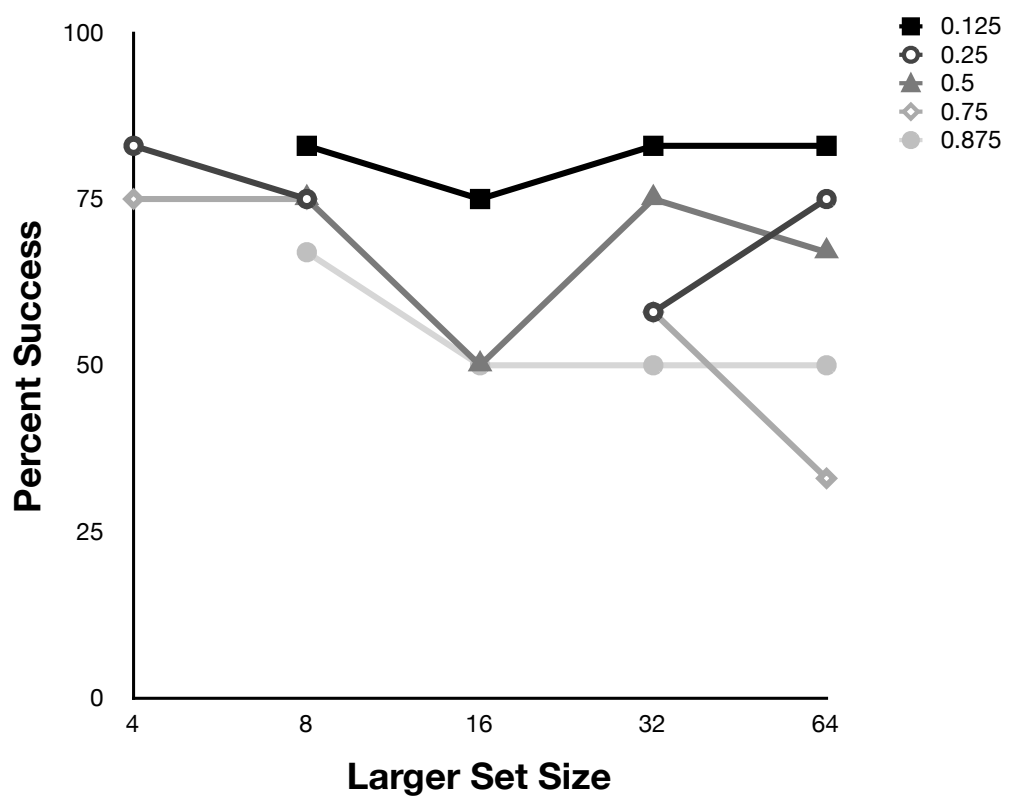

Figure 7. Percentage of correct trials as a function of the larger number of worms of the two quantities shown in trials of Experiments 2 and 3.

\section{Discussion}

Robins were capable of selecting the larger of two quantities when they were presented item-by-item and when both were presented simultaneously as visually accessible whole sets. These findings extend previous work by Hunt et al. (2008). In other ways, however, the findings are novel with respect to broader comparative RQJ research.

Consider the following themes in the literature. First, some studies have found that animals perform poorly when they have to process the sequential presentation of items so as to keep two sets of representations in working memory and compare quantities mentally (e.g., Abramson et al., 2011, with sea lions; Hanus \& Call, 2007, with apes). Second, psychophysical representations - based on Weber's law - are a clear signature of numerosity when animals are tested with quantities presented as a united set (e.g., Abramson et al., 2011). Third, whilst researchers have argued that the object-file tracking system is a better fit for capturing how animals represent small numbers irrespective of their ratio, the numerical representation involved here has a typical set size limit of 3 to 4 items (e.g., Feigenson et al., 2004; Hauser, 2000; Trick \& Pylyshyn, 1994). 
Robins' RQJs were different on all three counts. Robins actually show slightly higher accuracy in selecting the larger number over a range of values - small (1 to 4 ) and large (8 to 16) - when quantities were presented item-by-item (Experiment 1) than when quantities were presented simultaneously (Experiment 2). Further, while there was a general decline in accuracy in numerical discrimination with increasing objective number in each method of RQJ assessment, accuracy in both contexts were predicted by the total set size of the quantities and not ratio. And finally, in Experiments 1 and 2, robins were able to make accurate RQJs for values far exceeding the typical object-file system limit of around 3 to 4 items; robins were accurate even for comparisons of 7 vs. 8 (Experiments 1 and 2) and 14 vs. 16 (Experiment 1). At the very least, robins' object indexing system appears to operate over an extended number range of 1 to 16 , and robins are able to individuate up to 8 or more items in parallel. Whilst a detailed unpacking of robins' sophisticated numerical competence will require further research, there may be merit to Hunt et al.'s (2008) explanations vis-à-vis robins' food-caching ecology.

Initial RQJ testing by Hunt et al. (2008) indicated that robins chose the cache site with greater number with comparisons involving 1 vs. 2, 2 vs. 3,3 vs. 4,4 vs. 5 , and 4 vs. 8 , but chose randomly with 4 vs. 6 ; and 6 vs. 8 and 8 vs. 10 . While the levels of accuracy in these results differ from the present study, this is likely explained by methodology adapted in the experiments presented here specifically for improved examination of extremely large numbers. For example, one important alteration is a faster drop rate of $2 \mathrm{~s}$ per worm in the present study compared to $5 \mathrm{~s}$ in Hunt et al. (2008). This means that where a trial with 12 worms, for example, would be 60 seconds in duration in the Hunt et al. (Povinelli, 2000) study, it would require only 24 seconds to display in the present study (2.5 times faster). The advantage therein lies in being able to overcome difficulties with loss of interest or attention by the subject, particularly when presentation of prey requires longer than 40-60s, better maintaining full attention of the subject on the task at hand.

As a scatter-hoarding species, robins need to track each prey item to its endpoint - from being caught, stored, shifted, pilfered, and consumed. These physical inventories of stored food have high turnover rate due to perishability, are susceptible to pilfering by mates, fledglings and neighbours, and are maintained on permanent year-round territories with seasonal pressures (summer provisioning, 
winter resource competition). Robins' unusually high threshold for accuracy in quantity discrimination tasks complement evidence of neurological differences found not only between avian memory systems and mammals, but also between scatterhoarding birds and other birds (e.g., Clayton, 1994, 1995). Of course, similar RQJ experiments on wild birds that do not hoard food have yet to be conducted; attributing the advanced numerical skills of robins to food hoarding per se remains speculative. That said, birds' visual perception and spatial orientation are physiological systems that differ significantly from mammals and other animals (Cook, 2000), and are likely to play a significant role in shaping how even such fundamental core knowledge systems as number, geometry and object are evidenced in their natural behaviour. Geospatial information attached to items tracked by scatter-hoarding species could very well augment behavioural evidence of higher range object-based numerical system in such species the same way radio frequency identification (RFID) tags provide information enhancing a system's ability to track tagged items. For North Island robins, as scatter-hoarders, one ecological adaptation appears to be the use of a sophisticated number system which is able to precisely identify quantities larger than 3 or 4 .

It is important to point out that robins' RQJs across all three experiments could be influenced by cues other than numerousness, and are almost certainly likely to be affected by pilfering and hunting strategy specific to this species in ways that future experimentation will hopefully reveal in more detail. The present experiment did not control for cues such as differences in dropping duration (Experiment 1) or surface area (Experiments 2 and 3). The findings do not definitively rule out that robins are making decisions based on non-numerical cues to solve numerical problems. Nonetheless, it is encouraging that the findings in Experiment 1 replicate work by Hunt et al. (2008) where dropping duration was controlled for. Hunt et al. also showed that other sensory confounds (odour and sound) do not affect robins' RQJs. The attempt here was primarily to simulate problems robins might encounter in the wild wherein the item-by-item presentation might approximate subjects observing a conspecific dropping insect prey into a cache one-by-one and the whole set presentation might approximate subjects turning over leaf litter to discover prey items. As such, even though this design cedes certain experimental controls for gains in ecological validity, these findings still afford important comparisons with 
other studies that have used similar protocols to measure spontaneous RQJs (e.g., Helme, Call, Clayton, \& Emery, 2006) with sea lions, and (Hanus \& Call, 2007), with apes). Compared to those species where fewer subjects succeeded in the cognitively demanding item-by-item task context, robins were as accurate in RQJs with sequential presentation as they were with presentation of whole visible sets. As such, new findings have been uncovered about the representations that are at the root of robins' numerical abilities. Two systems appear to be at work when wild North Island robins perceive quantity: an approximate magnitude representation system which is effective for very large numbers and an object indexing system which remains effective at substantially higher thresholds than current theory posits.

Importantly, the current findings also support more than one mechanism underlying the ability to enumerate quantities in the North Island Robin. In Experiment 3, ratio was the only factor that significantly accounted for robins' RQJs involving very large values (16 to 64 ) presented simultaneously. Robins were able to discriminate between 32 and 64 prey items (0.50 ratio) but performance dropped to around chance level when 24 and 32 prey items were presented ( 0.75 ratio). With respect to large numbers that exceed the standard object-file range, mosquitofish have also been found to discriminate contrasts with ratios of 0.67 (8 vs. 12) but not ratios of 0.75 (9 vs.12) (Bisazza, Piffer, Serena, \& Agrillo, 2010). The developing precision of the analog magnitude representation system shown in human children also intersects with the ratio resolution in robins: Lipton \& Spelke (2003) show that 6 month-olds can discriminate a ratio of .50 (e.g., 8 vs 16) but not .67 (e.g., 8 vs 12) whereas 9-month-olds can discriminate a ratio of .66 (e.g., 8vs 12) but not .80 (e.g., 8 vs 10). As with Agrillo et al.'s mosquitofish $(2007,2008)$, resolution of the ratiobased large number discrimination in robins parallels the resolution of the ratiobased large number discrimination shown between that of a 9-month-old human infant and a human preschooler. Given the correspondence of cognitive signatures between species as diverse as robins, fish and human children's large number discrimination, these findings fit with the intriguing proposal that robins share with other species an analog magnitude system that is inherited from a common ancestor (Agrillo et al., 2008; Beran, 2008; Cantlon \& Brannon, 2006). 
CHAPTER 3: Study 2 - Quantity Discrimination Across Multiple Arrays 
Numerical competence in nonhuman species has been one of the more extensively researched cognitive domains to date. Perhaps one of the most fundamental and widely studied quantification abilities is relative numerousness judgments (RNJ), which has been demonstrated in nearly every species tested; from great apes, dolphins and elephants (Beran, 2007; Boysen \& Berntson, 1995; 2006; Irie-Sugimoto et al., 2009; Jaakkola, Fellner, Erb, Rodriguez, \& Guarino, 2005; Kilian, Yaman, von Fersen, \& Güntürkün, 2003; Perdue, Talbot, Stone, \& Beran, 2012) to pigeons, salamanders and even beetles (Carazo et al., 2009; Emmerton, 1998; Krusche, Uller, \& Dicke, 2010; Uller et al., 2003). Studies where subjects are asked to discriminate different quantities present stimuli in a broad spectrum of manners using multiple sensory modes such as auditory, visual (Beran, 2012; Kilian, von Fersen, \& Güntürkün, 2005; McComb, Packer, \& Pusey, 1994), multiple media types: food items, tokens (Addessi et al., 2008; Beran, Evans, \& Hoyle, 2011), shapes on a computer screen (Honig \& Matheson, 1995; Krusche et al., 2010) or paper (Bogale, Kamata, Mioko, \& Sugita, 2011; Emmerton, 1998), and multiple methods: sequential, simultaneous, contiguous or dispersed presentations.

Many quantity discrimination studies, by nature of the choice task, present different quantities in visible 'arrays', though this can describe anything from an assortment of food items scattered in a defined space (Study 1) (Garland, Low, \& Burns, 2012; Krusche et al., 2010) to ordered grids of items controlled for density and surface area (Tomonaga, 2008). To date, however, research comparing hidden quantities of items have consistently been presented by being placed continuously into the same visually obscured location (Beran, Beran, Harris, \& Washburn, 2005; Hanus \& Call, 2007; Perdue et al., 2012).

There are also studies that utilise an array in which items are hidden in select locations, so that the locations and quantity of items is not visible to the subject. The majority of spatial memory tasks broaching number do so by examining the retrieval or identification of objects hidden within a single array (Sanford \& Clayton, 2008; Scheid \& Bugnyar, 2008), which is sometimes rotated in orientation (Beran, 2006; Beran, Beran, \& Menzel, 2005; Miller, Rayburn-Reeves, \& Zentall, 2009). Such studies are primarily aimed at short or long term memory of object locations, where the quantity retrieved is sometimes used as a measure of success (Bednekoff \& Balda, 1996; Gould, Ort, \& Kamil, 2012). While there is a large and growing body of 
research addressing spatial memory in many ways, little or none to date has addressed numeracy in terms of quantity discrimination between multiple arrays of hidden items.

Cache recovery is an area of spatial memory research that has investigated a range of ecological and cognitive influence on the success and accuracy rates of recovery of caches in a number of avian species, including for example coal tits, Parus ater (Agrillo et al., 2004), chickadees, Parus atricapillus (Baker et al., 1988; Hitchcock \& Sherry, 1990), Clark's nutcrackers, Nucifraga columbiana (Balda \& Kamil, 1992; Bednekoff \& Balda, 1996; Bednekoff et al., 1997; Dunlap, Chen, Bednekoff, Greene, \& Balda, 2006), jackdaws, Corvus monedula (Vander Wall \& Balda, 1981) and ravens, Corvus corax (Bugnyar \& Kotrschal, 2002), for example. Such a task certainly involves location and retrieval of items in an array, in a broad sense; either distributed throughout a given area in the field, or within an aviary room or specific apparatus (such as an ice cube tray). As the vast majority of such studies aim to observe and manipulate variables involved in success rates in recovering a cache of items hidden by the bird itself, such studies rarely appear to involve recovery of items hidden by the experimenter.

The present study presents two discrete sets, each distributed across an array, where no more than one item is visible at a time. The data we obtain from these experiments will help us to examine two questions. First, this experiment will look at whether New Zealand robins can compare two sets of items, viewed one at a time and then hidden, and spatially distributed across an array. Second, it will examine whether ratio, numerical difference between quantities, and single and combined sums of quantities influence performance.

\section{Methods}

Subjects. Five individual adult male robins participated in this experiment. Each subject was identified with the unique combination of coloured bands on the bird's legs. This study was conducted in native New Zealand forest within the Zealandia Sanctuary. The sanctuary is located on the southern tip of the North Island of New

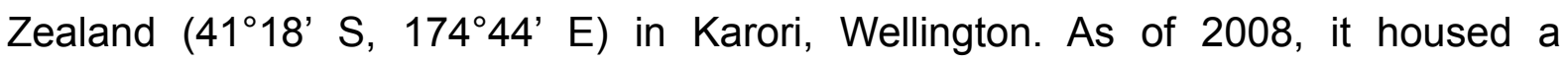
population of approximately 150 colour-banded birds, and a total population estimated to be just under 600 birds (McGavin, 2009). Robins used in trials were 
located auditorily and visually along a series of footpaths or transects traversing the Karori Sanctuary.

Materials. RQJs were examined by presenting subjects with mealworms (Tenebrio molitor larvae) in artificial caches comprising of two separate wooden planks, each containing an array of seven shallow, sunken wells drilled into the wood and lined in clear plastic (see Figure 8). Three wells were in the row nearer the robin and four behind that row; rows were offset so that all wells were visible at once. Each wooden plank measured approximately $2 \mathrm{~cm}$ thick, $13.5 \mathrm{~cm}$ wide and $31 \mathrm{~cm}$ long, and was natural unpainted pine. Each well measured approximately $0.5 \mathrm{~cm}$ deep, $5 \mathrm{~cm}$ in diameter, and were spaced approximately $1.5 \mathrm{~cm}$ apart. The planks were spaced approximately $45 \mathrm{~cm}$ apart, with variation due to vegetation and terrain. The wells were each covered during the display with two sliding covers consisting of thin brown pieces particleboard measuring $6 \mathrm{~cm}$ across and $31 \mathrm{~cm}$ long. Upon completion of filling the wells, each array was completely covered with an opaque dark green canvas cloth, measuring $13.5 \mathrm{~cm}$ wide and $31 \mathrm{~cm}$ long. A pair of stainless steel tweezers was used to transfer the mealworms from a small, opaque white plastic container into the wells.

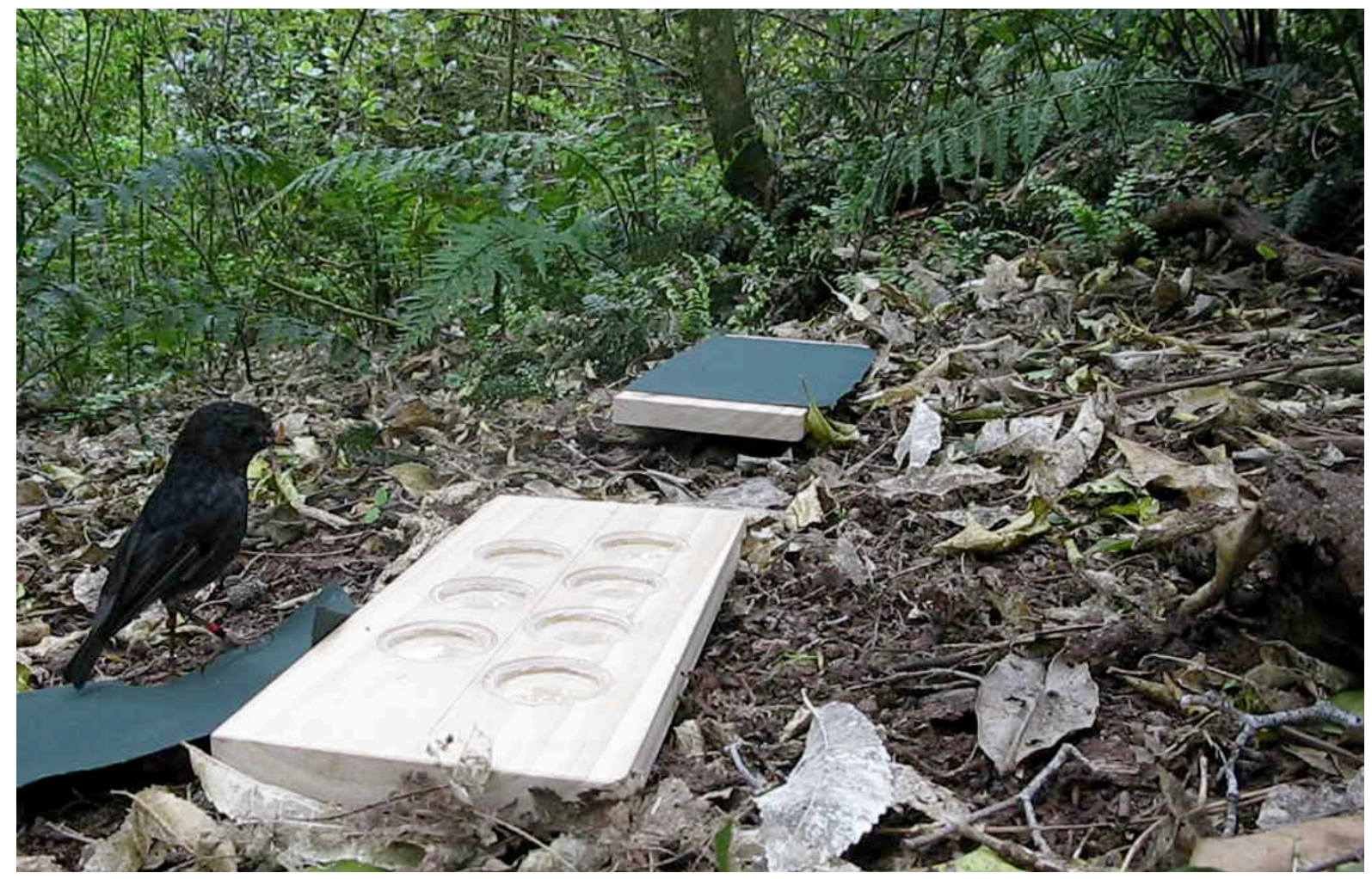

Figure 8. A robin makes a choice between two arrays. 
Procedure. Mealworm prey was presented to wild robins in an experimental arena containing two experimental arrays of caches (see Appendix $C$ for links to example videos). First, the arrays and sliding covers were placed in an appropriate location within the robin's territory, and the container of mealworms placed equidistant between the two arrays, $0.5 \mathrm{~m}$ towards the experimenter. The experimenter then used the tweezers to take mealworms from the plastic container and placed them randomly in 1-5 of the 7 wells on each array. For the wells left empty, the same motion was made with the tweezers, but without containing a mealworm. As each well was either filled or touched with empty tweezers, the experimenter covered the well immediately with the particleboard slider, so that only wells being filled and unfilled empty wells were within view of the subject. When all 7 wells were covered with the sliders, the green canvas cloth was placed over them, and the sliders were pulled out from beneath the cloth, allowing the subject to easily access all 7 wells when the cloth was removed. Subjects were then allowed to approach and remove one of the two cloths (by pulling it off with their beaks), and retrieve mealworms. Robins were allowed to retrieve and consume or cache the mealworms found regardless of the choice made, but only from one of the two caches.

The order of treatments were randomised in an attempt to control for observational learning. Six different quantity comparisons were displayed to each subject: 1 vs. 2,1 vs. 3,2 vs. 4,2 vs. 3,1 vs. 5 , and 2 vs. 5 . Three birds responded to 9 repetitions of each comparison (54 total trials per individual), and two birds responded to 7 repetitions of each comparison (42 total trials per individual). Trials were conducted within the forest territory of each bird.

\section{Results}

In this experiment, first the overall results are examined, and then the data is analyzed by season, comparing the data collected during nesting season with data collected during winter. During nesting season, males spend the predominate portion of their time provisioning their mate, nestlings and fledglings.

Overall results. Overall, each robin performed significantly above chance in choosing the larger quantity $(p<.005$, binomial test; OM-RK $-71.07 \%$; RW-OM 78.57\%; RY-fPM - 70.37\%; RM-OB - 70.37\%; OM-YK - 73.81\%) (see Figure 9). Individually, each robin performed above chance in each number comparison with 
the exception of RY-fPM, who performed $2 \mathrm{v} 3$ below chance $(45 \%)$ overall (see Figure 9). A linear stepwise regression was conducted, examining item ratio, numerical difference, total quantity displayed, the smaller of two quantities, and larger quantity as independent variables, weighted by trial count across all animals. The dependent variable was 'success', or the percent of successful choices made by each subject for each comparison.

Only 'smaller quantity', or the smaller of the two quantities displayed, was found to be a significant predictor of RQJ performance, $\left(F_{1,28}=4.870, p=0.036, B=\right.$ -0.385, $d f=29$ ). A Pearson Correlation also showed 'smaller quantity' significantly correlated with 'success' $(r(28)=0.683, p=0.030)$. Ratio $(p=0.744)$, numerical difference $(p=0.498)$, total quantity $(p=0.498)$ and larger quantity $(p=0.498)$ were all excluded variables. One of the five robins showed a significant side bias towards the array on the right side from the perspective of the participating subject, (RY-fPM $64.8 \%, p<.05$, two-tailed binomial test). The other four robins (OM-RK $-57.4 \%$; RW-OM - 47.6\%; RM-OB - 57.4\%; OM-YK - 61.9\%) did not differ from chance ( $p>$. 05, two-tailed binomial test).

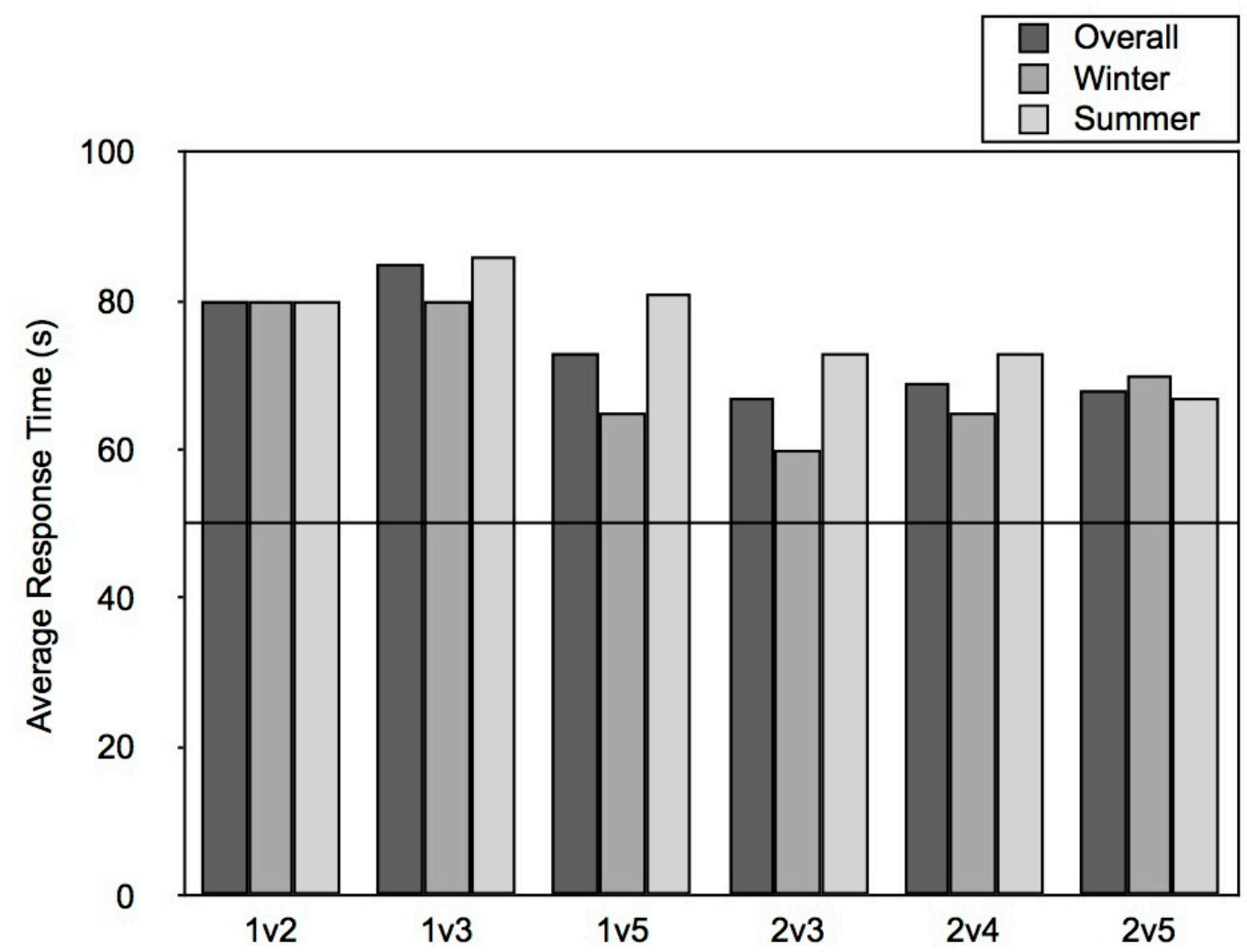

Figure 9. Percentage success across all participants for all 6 number comparisons, overall, in winter, and in summer. 
Seasonal Differences. Results for trials were collected during two behaviourally distinct seasons for robins: winter (July 2011) and summer (Oct 2010-Feb 2011), which is nesting season. Looking at individual subject across all summer trials, each robin performed significantly above chance in choosing the larger quantity $(p<.05$, binomial test; OM-RK - 80\%; RW-OM - 83.33\%; RY-fPM - 70\%; RM-OB - 70\%; OM-YK - 83.33\%). Robins performed at RQJs significantly $(N=21, p=.000)$ above chance across all six number comparisons (see Figure 9), ranging between $66.7 \%$ (2v5) to $90.5 \%$ (1v3), with $\mathrm{N}=21$ for each number comparison across all 5 birds. Three robins completed a total of 30 trials each (5 for each comparison), and two robins completed a total of 18 trials each (3 for each comparison).

Looking at individual subject across all winter trials, one robin performed significantly above chance in choosing the larger quantity $(p<.05$, binomial test; RW-OM $-75 \%$ ), two robins performed above chance, but only approaching marginal significance ( $p=.064$, binomial test; RY-fPM - 70.83\%; RM-OB - 70.83\%), and two robins did not perform significantly above chance $(p>.05$, binomial test; OM-RK 67.77\%; OM-YK - 67.77\%). Robins performed at RQJs significantly ( $N=20, p=$. 000) above chance across all six number comparisons (see Figure 9), ranging between $60.0 \%(2 \mathrm{v} 3)$ to $80.0 \%$ (1v3 and $1 \mathrm{v} 2$ ), with $\mathrm{N}=20$ for each number comparison including 5 birds. All five robins completed a total of 24 trials each (4 for each comparison).

A linear regression was conducted, examining the following independent variables: item ratio in combination with total, smaller, and larger set size, as well as numerical difference in combination total, smaller, and larger set size; each weighted by trial count. The dependent variable was 'success', or the percentage of successful choices made by each subject for each comparison. None of the variables emerged as significant, either for the winter or nesting season trials. A Pearson Correlation also shows no significant correlations between any independent variables and 'success'. Results indicate no significant differences in performance across season.

\section{Discussion}

This experiment presents a challenging cognitive task, requiring attention both to given quantities of hidden items, as well as spatial location. The data further illustrates an interesting difference in the performance of the North Island robin in 
quantity tasks with comparison to most other species, in that responses did not predictably vary with the ratio of items. This characteristic emerged in previous quantity experiments as well (see Chapter 2) (Garland et al., 2012; Hunt et al., 2008). An experiment using added dimensions of lapsed time prior to retrieval and obscuring visual access also lowered success rates of a discrimination task with added cognitive demand (Armstrong, 2011).

While the percentage success of this experiment was above chance (66-85\%), it was below performance for the the smallest comparisons presented and hidden in a single location in hidden trials of Study 1 (1v4, 3v4, 1v8; 75-100\% success). Robins appear more influenced by the total number of prey, as total (as well as the smaller and larger quantities) did appear significant on a forced entry regression. For example, if ratio was predictive of outcome, it would be expected that robins perform most successfully at $1 \mathrm{v} 5$; if it played no role, they might perform with lower success at $1 \mathrm{v} 5$ than $1 \mathrm{v} 3$, where there is a lower total number of worms, but less numerical distance. Robins also appeared to have a side bias for the left side of the visual field; another indicator differentiating this experiment with previous, less cognitively demanding discrimination tasks.

The smaller of the two numbers presented - whether it is 1 or 2 - appears most significant variable for robins (i.e. they are much more likely to choose correctly if the smaller number is 1 than 2). This is interesting, as it may be indicative of a strategy employed for such a cognitively demanding task as the one presented here, and account for absence of ratio as a predictor despite lower success levels in choosing the greater quantity. This could further support research showing a distinction between single-plural sets in conditions that do not promote distinctions based on an approximate magnitudes (Barner, Wood, Hauser, \& Carey, 2008). In a study by Barner et al. (2008), rhesus monkeys were able to distinguished between singular and plural sets $1 \mathrm{v} 2,1 \mathrm{v} 5$, but not two plural sets $2 \mathrm{v} 3,2 \mathrm{v} 4,2 \mathrm{v} 5$. Robins' lower levels of success with plural sets could provide evidence for a more basic singular-plural distinction in lieu of deploying mechanisms that might work more successfully at larger numbers, like ratio, or the more precise distinctions in tasks requiring less simultaneous spatial memory. 
CHAPTER 4: Study 3 - Addition and Subtraction 
Research on cognition in certain songbirds has challenged the belief that primates and human infants are the only non- or pre-linguistic animals with sophisticated cognitive abilities (Bugnyar \& Heinrich, 2006; Emery et al., 2004; Taylor, Hunt, Medina, \& Gray, 2009). There is still a limited understanding of avian numerical reasoning (Emmerton, 1998; Pepperberg, 2006; Roberts, Coughlin, \& Roberts, 2000) and the extent to which this core system of knowledge may have evolved independently from the cognitive skills of primates and humans (Beran, 2006, 2008; Cantlon \& Brannon, 2006; Feigenson et al., 2004; Hauser et al., 2004; Wynn, 1992, 1995; Wynn et al., 1998). This study focuses on establishing the New Zealand robin as one promising model system for understanding the evolutionary precursors of human mathematical ability. This experiment examines the ability to spontaneously compute (i.e., without training) arithmetic operations over small numbers of objects.

A variety of studies show that infants can perform basic, non-symbolic arithmetic computation, such as addition and subtraction of items (Barth et al., 2006; McCrink \& Wynn, 2004; Wynn, 1992). Using the Violation of Expectancy (VoA) paradigm, where in some scenarios an 'impossible' outcome is revealed, Wynn (1992) demonstrated that five-month-olds successfully responded to simple arithmetic operations. Infants were shown a certain number of toys as they were placed behind a screen, and in some instances the outcome was expected (e.g. 1+1=2), and in other cases the number revealed was unexpected (e.g. $1+1=1$ ), and were found to look longer at outcomes where expectation was violated. There remains debate over whether infants are capable of perceptual processing of numerical information itself, or are responding to other features. A replication of the study three years later (Simon, Hespos, \& Rochat, 1995) suggested the possibility that outcomes could be based on knowledge of physical object behaviour rather than underlying arithmetical ability, as the infants in this study, while responding by looking longer at mathematically incorrect outcomes, did not look longer when the type of object hidden was switched. An alternative explanation is that while infants are capable of representation of simple arithmetic operations, the objects of such operations are stripped of their nonnumerical features (Rugani, Fontanari, Simoni, Regolin, \& Vallortigara, 2009). Cohen and Marks (2002) however, suggest based on further experimentation that the infants in Wynn's study might be responding with familiarity preferences along with 
the tendency to look longer at more items. While it has been shown that infants do respond with increased looking time to perceptually novel displays, either in item type (2006) or number (Slater, Bremner, Johnson, \& Hayes, 2010), it does not rule out the use of arithmetic operations such as addition or subtraction. In contrast, it supports that outcomes in addition/subtraction experiments persisting despite such preference cannot be simply explained because of such familiarity alone. Further evidence shows that infants appear able to add and subtract over numbers that exceed object-tracking limits (McCrink \& Wynn, 2004), supporting representation of numerical computation.

A number of different animal species have also shown the ability to perform simple addition and subtraction of small numbers, including rhesus monkeys, Macaca mulatta (Hauser et al., 1996; Hauser, Tsao, Garcia, \& Spelke, 2003; Sulkowski \& Hauser, 2001), cotton-top tamarins, Sanguinus oedipus (Uller, Hauser, \& Carey, 2001), lemurs (Eulemur fulvus, E. mongos, Lemur catta and Varecia rubra) (Santos, Barnes, \& Mahajan, 2005) and domestic dogs, Canis familiaris (West \& Young, 2002). Chimpanzees (Pan troglodytes) have been shown capable of summing somewhat larger quantities (such as $3+4+1$ or $2+2+3$ ) (Beran, 2001). In Flombaum et al.'s study (Flombaum, Junge, \& Hauser, 2005), rhesus monkeys also successfully recognised addition of larger numbers (such as $4+4=8$ ) when using lemons in a VoE study.

Exploration of arithmetic operations in avians has been somewhat less investigated, however. In a study by Brannon et al. (2001), pigeons were asked to compare a constant number with the number remaining after a numerical subtraction, in a forced-choice testing phase following training. In one phase, a timeleft paradigm was presented, and in another a number-left paradigm, where for each it is assumed subjects are using memory to subtract their count of the tally of flashes shown. A study examining addition of numbers up to 6 arose spontaneously when Alex, a grey parrot (Psittacus erithacus) (Pepperberg, 2006), spontaneously answered numerical identification questions posed to another bird by tallying the clicks after each repeated enquiry. The resultant study revealed Alex was successfully able to sum numbers up to six (e.g. $5+1,2+4,3+2$, etc.) by responding verbally with the correct total number when briefly shown two sets of items each covered with cups. 
Addition and subtraction of items has also been recognised in newborn chicks (Gallus gallus) (Rugani et al., 2009). The chicks were first imprinted on a group of 5 plastic balls, and then exposed to a brief training session in which the chick was familiarised with the experimental arena and trained to follow the same type of plastic ball after being moved behind an occluding screen. In the testing phase of the final experiment, after two initial sets of five balls (e.g. 2 and 3, or 4 and 1) were hidden behind two screens, some elements were visibly transferred from behind one screen to behind the other; for example, creating a 4-2 and 1+2 condition, where the screens began with 4 and 1 . Chicks successfully chose the larger set of objects after the addition/subtraction event regardless of directional cues due to initial or final movement of the objects. Such pronounced results in chicks only several days old provides compelling evidence for representation of simple arithmetic operations as part of system of number representation in vertebrates.

While the rhesus monkeys presented with a choice task in Sulkowski's (2001) and Flombaum \& Santos' (2005) studies were free-ranging, very little experimentation focusing specifically on arithmetic operations has been done with wild populations. This study presents a population of wild North Island robins with a Violation of Expectancy ( $\mathrm{VOE}$ ) task in two separate experiments. Experiment 1 presents robins with trials that are in some cases numerically congruent, and in other cases numerically incongruent. Experiment 2 presents robins with trials that are categorically incongruent or categorically congruent. The data from these experiments will attempt to examine whether North Island robins respond with different search times to the addition or subtraction of small numbers of prey items.

\section{Methods}

Subjects. A total of 17 individual robins participated in this study, 7 birds in the first experiment and 10 birds in the second experiment. No single subject participated in more than one of the two experiments described below. Each subject was identified with the unique combination of coloured bands on the bird's legs. Some of the robins used in this study had prior experimental history. This study was conducted in native New Zealand forest within the Zealandia Sanctuary. The sanctuary is located on the southern tip of the North Island of New Zealand (41 ${ }^{\circ} 18^{\prime}$ S, $174^{\circ} 44^{\prime}$ E) in Karori, Wellington. As of 2008 , it housed a population of 
approximately 150 colour-banded birds, and a total population estimated to be roughly 600 birds (McGavin, 2009). Robins used in trials were located auditorily and visually along a series of footpaths or transects traversing the sanctuary.

Apparatus. Trials were performed by presenting subjects with mealworms (Tenebrio molitor larvae) or waxworms (Galleria mellonella larvae) in a wooden encasement designed for Violation of Expectancy (VoE) testing (see Figure 10). The box measured $9.5 \mathrm{~cm}$ wide, $4 \mathrm{~cm}$ tall, and $17 \mathrm{~cm}$ long, with a thin, particleboard slider protruding from one side which extends out when pulled and slides into the box when pushed. This slider attaches to an upper compartment comprising a clear Petri dish measuring $6 \mathrm{~cm}$ in diameter mounted on the inside of the box. The side of the Petri dish facing the inside of the box into which it can be pulled is covered in opaque black tape so that only the contents of the dish is visible when this sliding compartment is pushed into the box. A hole measuring $6 \mathrm{~cm}$ in diameter on the surface of the box allows the subject to see either the sliding compartment $(1 \mathrm{~cm}$ deep), when it is pushed into the box. The well below is accessible when the sliding compartment is pulled out fully, and lined with the same type and size plastic petri dish. The plastic lining of each well served to prevent the mealworms from being able to climb the rough wooden surface of the encasement. The well was covered with a circular brown leather flap approximately $7 \mathrm{~cm}$ across. Mealworms and waxworms were placed in the apparatus with stainless steel tweezers, to enable the clearest possible view of the prey.

Procedure. In both experiments, the VoE box (see Figure 10) was used with both the upper and lower compartments filled, regardless of whether the outcome was expected (items were the same in number or type) or unexpected (items differed in number or type). In that manner, all robins observed prey being dropped only into the lower compartment, and were only able to retrieve it from the upper compartment (see Appendix $\mathrm{C}$ for links to example videos). Any change in response based on a perceived difference in the depth of the well, or multiple insects being contained therein, should be responded to both for expected and unexpected events. 


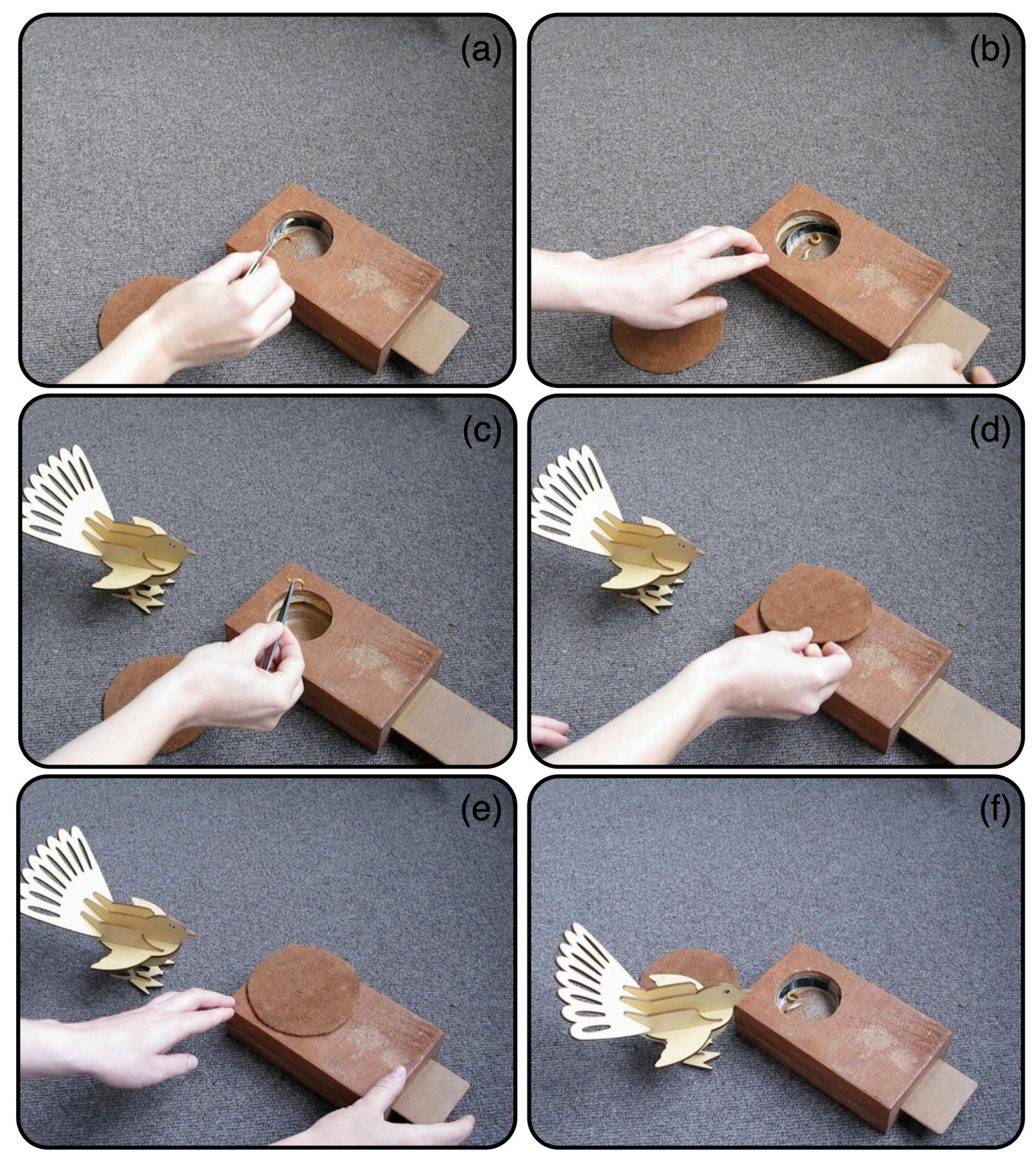

Figure 10. The VoE apparatus: Out of the bird's view, the upper compartment is slid in, (a) insects placed inside and (b) the compartment is slid out, hiding it. In the bird's view, in the lower compartment (c) insects are placed inside, (d) the lid covers the well, $(e)$ the drawer is slid in, and (f) the bird uncovers the upper compartment. ${ }^{3}$

${ }^{3}$ The wooden bird in this demonstration is meant to represent the participating robin as it might view and interact in a given trial, in order to make all the parts of the apparatus better visible. The proximity of the bird to the apparatus is not reflective of an actual trial scenario. 
Robins were also exposed to neutral controls in both experiments, in order to account for the natural curiosity for novelty characteristic of this naive species. Neutral exposures consisted of displaying only the VoE box and leather flap without running any trial, and measuring the same variables - general attentiveness, search time and frequency of pecking, but without running any numerical or categorical trial, or placing any prey items inside or around the box. For all trials and control exposures, a trial was considered 'ended' when the robin remained more than a meter away from the apparatus for longer than 20 seconds.

Experiment 1: Item number. Robins were shown 8 different hiding events in randomised order, and found 4 numerically congruent and 4 numerically incongruent (see Table 3). In this experiment, only mealworms (Tenebrio molitor) were used.

Table 3. Congruent and Incongruent conditions presented in Experiment 1, altering item number.

\begin{tabular}{ll}
\hline & Experiment 1 \\
\hline Congruent & Incongruent \\
\hline $1+0=1$ & $1+1 \neq 1$ \\
$1+1=2$ & $2-0 \neq 1$ \\
$2-1=1$ & $3-0 \neq 2$ \\
$3-1=2$ & $3-1 \neq 1$ \\
\hline
\end{tabular}

Out of view of the experimental subject, the sliding compartment of the VoE box was first loaded with the number of worms intended for the robin to find (Figure 10a), and the sliding compartment was pulled fully out so that the contents was not visible (Figure 10b). The VoE box was then placed so that it was clearly visible to the subject, within a $1-3 \mathrm{~m}$ distance of the robin's viewing position. Worms were then 'added' by dropping them in clear view of the robin into the empty bottom compartment of the VoE box, first displaying the worm clearly for approx. 4s by holding it in tweezers directly over the well, and then dropping each in with approximate 10 s between worms (Figure 10c).

In some events worms were 'subtracted' by removing the worm from the well with the same approximately 10s gap, and holding it again in tweezers for approx. 4s 
before replacing the worm into the small white $35 \mathrm{~mm}$ film canister holding the remainder of the worms. The trials were only conducted with robins when they were on or very close to the ground, so that they were unable to see the final total of worms present in the bottom compartment of the box, due to depth. The experimenter then covered the well with the leather cover (Figure 10d), and pushed the sliding compartment fully in, so that the upper compartment with the pre-loaded worms were the ones visible to the robin upon retrieval (Figure 10e). Lastly, robins were given the opportunity to approach and uncover the well, and retrieve a number of mealworms either consistent or inconsistent with the number of mealworms they observed being added or removed (Figure 10f).

Experiment 2: Item category. In this experiment, both mealworms (Tenebrio molitor larvae) and waxworms (Galleria mellonella larvae) were used. Robins were shown 6 different hiding events in randomised order, and found 4 categorically congruent and 4 categorically incongruent (see Table 4).

Table 4. Congruent and Incongruent conditions presented in Experiment 2, altering item category, where ' $m$ ' is mealworm, and ' $w$ ' is waxworm.

\begin{tabular}{ll}
\hline \multicolumn{2}{c}{ Experiment 2} \\
\hline Congruent & Incongruent \\
\hline $1 \mathrm{~m}+0 \mathrm{~m}=1 \mathrm{~m}$ & $1 \mathrm{~m}+1 \mathrm{w} \neq 2 \mathrm{~m}$ \\
$1 \mathrm{~m}+1 \mathrm{~m}=2 \mathrm{~m}$ & $1 \mathrm{~m}+1 \mathrm{w} \neq 2 \mathrm{w}$ \\
$1 \mathrm{~m}+1 \mathrm{w}=1 \mathrm{~m} 1 \mathrm{w}$ & $1 \mathrm{~m}+1 \mathrm{~m} \neq 1 \mathrm{~m} 1 \mathrm{w}$ \\
\hline
\end{tabular}

The sliding compartment of the VoE box was first loaded with the number of worms intended for the robin to find, out of view of the experimental subject (Figure 10a), and the sliding compartment was pulled fully out so that the contents was not visible (Figure 10b). The VoE box was then placed so that it was clearly visible to the subject, within a 1-3m distance of the robin's viewing position. Worms were then 'added' by dropping them in clear view of the robin into the empty bottom compartment of the VoE box, first displaying the worm clearly for approx. 4s by holding it in tweezers directly over the well, and then dropping each in with approximate 10 s between worms (Figure 10c). The trials were only conducted with 
robins when they were on or very close to the ground, so that they were unable to see the final total of worms present in the bottom compartment of the box, due to depth. The experimenter then covered the well with the leather cover (Figure 10d), and pushed the sliding compartment fully in (Figure 10e), so that the upper compartment with the pre-loaded worms were the ones visible to the robin upon retrieval. Lastly, robins were given the opportunity to approach and uncover the well, and retrieve a combination of worms either consistent or inconsistent with the category of worms they observed being added or removed (Figure 10f).

\section{Results}

A video analysis was performed looking at 6 different dimensions of response behaviour: First, attendance duration (Attend) - the amount of time the subject spent within 1 meter of the apparatus, generally attending even when not actively searching. Second, search duration (Search) - the total amount of time the robin spent actively examining the apparatus or leather cover. Third, pecking frequency (Pecks) - the number of times the subject pecked with its beak at any part of the apparatus. The data was also analysed jointly (across both Experiments 1 and 2) looking at the destination of the prey obtained by the robin, and whether it was eaten immediately or removed for caching or provisioning, to examine any differences that might be seen in response duration as a result.

Experiment 1: Item number. This experiment involved hiding events using only one type of prey (mealworms), in which an experimenter displayed an addition or subtraction event where the robin found prey that was either consistent or inconsistent in number with the prey seen being dropped into the box. Six males and one female participated in this experiment. On average, robins measured higher on all three measures in incongruent than congruent trials (Figure 11).

A One-Way ANOVA was used to compare means between conditions: congruent trials $(\mathrm{N}=28)$, incongruent trials $(\mathrm{N}=28)$, and neutral exposures $(\mathrm{N}=14)$. All three response dimensions were all higher on average in incongruent trials (Attend = 52.46s, Search $=42.96 \mathrm{~s}$, Pecks $=6.82$ ), than neutral exposures $($ Attend $=50.93 \mathrm{~s}$, Search $=36.71 \mathrm{~s}$, Pecks $=4.71)$, which were in turn higher than congruent trials $($ Attend $=27.68 \mathrm{~s}$, Search $=23.39 \mathrm{~s}$, Pecks $=1.25)$. Of these, responses were significantly different: attendance duration $(F(2,67)=4.226, p=.019)$, search 
duration $(F(2,67)=4.623, p=.013)$, and pecking frequency $(F(2,67)=5.678, p=$. 005).

Independent-Samples T-Tests were used to compare means between each condition. Congruent and incongruent trials were significantly different in all three measures: attendance (mean $\Delta 24.78 \mathrm{~s} ; t(54)=-2.759, p=0.005$ ) and search (mean $\Delta$ 19.57s; $t(54)=-3.117, p=0.002)$, and pecking (mean $\Delta 5.57 ; t(54)=-3.204, p=$ 0.000 ), as were neutral and congruent trials: attendance (mean $\Delta 23.25 \mathrm{~s} ; t(40)=$ -2.657, $p=0.010$ ) and search (mean $\Delta$ 13.32s; $t(40)=-2.013, p=0.007$ ), and pecking (mean $\Delta 3.46 ; t(40)=-3.127, p=0.000)$. While neutral exposures were on average lower than incongruent trials, they were not significantly so in any of the three measures: attendance (mean $\Delta 1.53 \mathrm{~s} ; t(40)=0.114, p=0.727$ ) and search (mean $\Delta 6.25 \mathrm{~s} ; t(40)=0.659, p=0.807)$, and pecking $($ mean $\Delta 2.11 ; t(40)=0.823, p$ $=0.106)$.

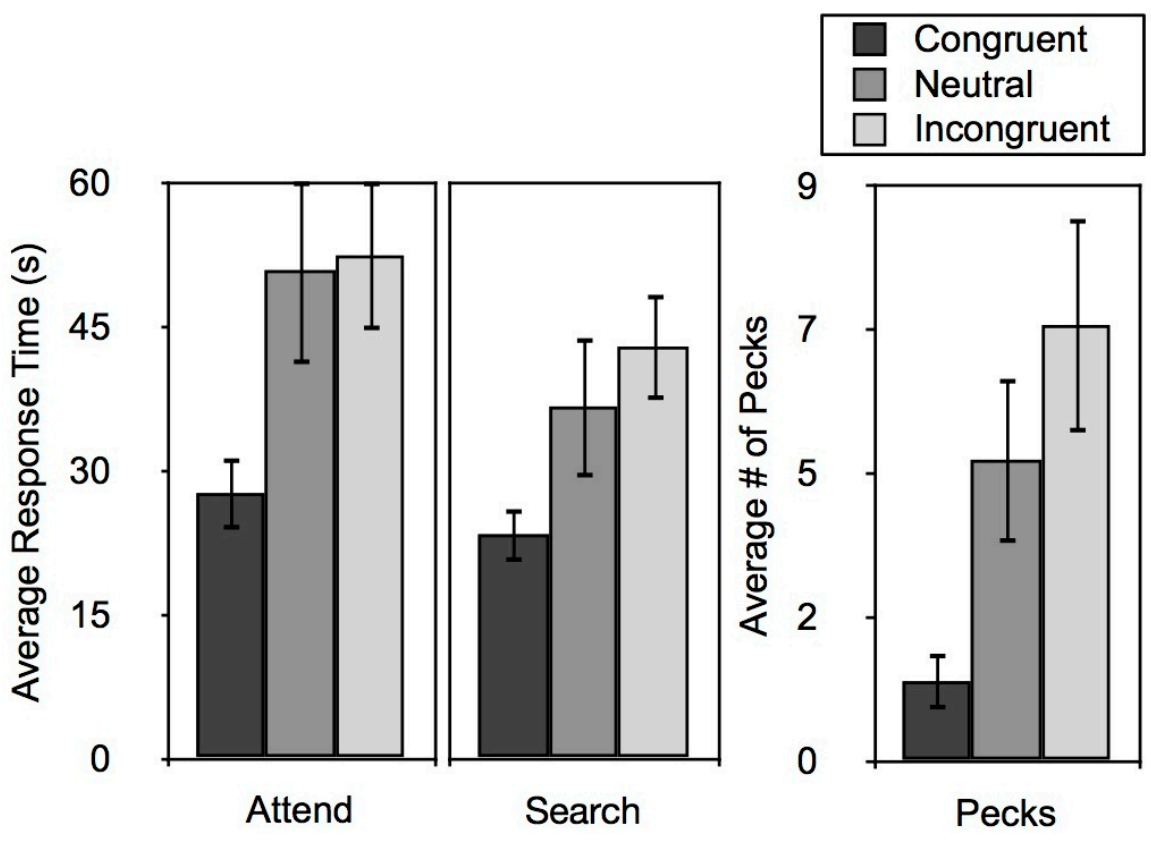

Figure 11. Experiment 1 (Item number) - Average response in search and attendance time and number of pecks.

Experiment 2: Item category. This experiment involved hiding events using two types of prey - mealworms and waxworms. An experimenter displayed an addition or subtraction event where the robin found prey that was either consistent or inconsistent in type with the prey seen being dropped into the box. Eight males and 
two females participated in this experiment. On average, robins measured higher on all three measures in incongruent than congruent trials (Figure 12).

A One-Way ANOVA was used to compare means between conditions: congruent trials $(\mathrm{N}=30)$, incongruent trials $(\mathrm{N}=30)$, and neutral exposures $(\mathrm{N}=20)$. On average, all three response dimensions were all highest in incongruent trials (Attend $=71.13 \mathrm{~s}$, Search $=52.90 \mathrm{~s}$, Pecks $=4.93)$. Neutral exposures again, on average, fell between the values for congruent and incongruent trials (Attend $=55.25 \mathrm{~s}$, Search $=38.40 \mathrm{~s}$, Pecks $=4.80)$. Values for congruent trials were all lowest of the three conditions (Attend $=37.13 \mathrm{~s}$, Search $=27.07 \mathrm{~s}$, Pecks $=1.00)$. Of these, all three response dimensions were again significantly different between conditions in all three measures, including attendance duration $(F(2,77)=3.522, p=.0349)$, search duration $(F(2,77)=3.5403, p=.034)$, and pecking frequency $(F(2,77)=5.679, p$ $=.005)$.

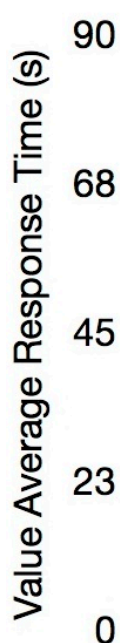

0

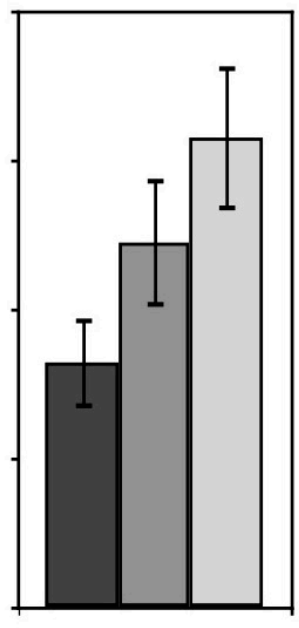

Attend

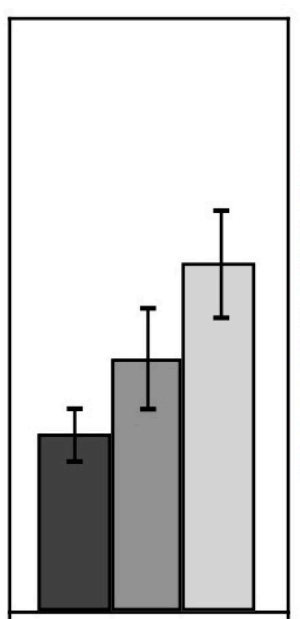

Search

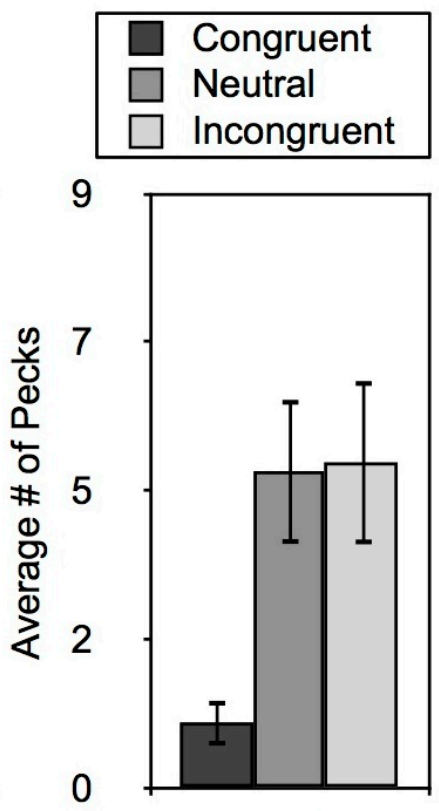

Peck

Figure 12. Experiment 2 (Item category) - Average response in search and attendance time and number of pecks.

Independent-Samples T-Tests were used to compare means between each condition. Congruent and incongruent trials were significantly different in all three measures: attendance (mean $\Delta 34.00 \mathrm{~s} ; t(58)=-2.557, p=0.048$ ) and search (mean $\Delta$ 25.83s; $t(58)=-2.619, p=0.012)$, and pecking (mean $\Delta 3.93 ; t(58)=-3.045, p=$ 0.000), while between neutral and congruent trials, only pecking frequency was 
significantly different: attendance (mean $\Delta$ 18.12s; $t(48)=-1.563, p=0.724$ ) and search $($ mean $\Delta 11.33 \mathrm{~s} ; t(48)=-1.313, p=0.124)$, and pecking (mean $\Delta 3.80 ; t(48)=$ -3.816, $p=0.001$ ). While neutral exposures were on average lower than incongruent trials, they were not significantly so in any of the three measures: attendance (mean $\Delta$ 15.88s; $t(48)=0.990, p=0.171)$ and search $($ mean $\Delta 14.50 \mathrm{~s} ; t(48)=1.154, p=$ $0.315)$, and pecking (mean $\Delta 0.13 ; t(48)=0.075, p=0.204)$.

Prey destination. Taken together, the prey destination - whether it was eaten, taken away for caching or provisioning, or both occurred (once with each worm) played a significant role in the response duration (see Figure 13), and much longer response durations when the worm was eaten immediately. There were no trials in which prey was left in the VoE box.

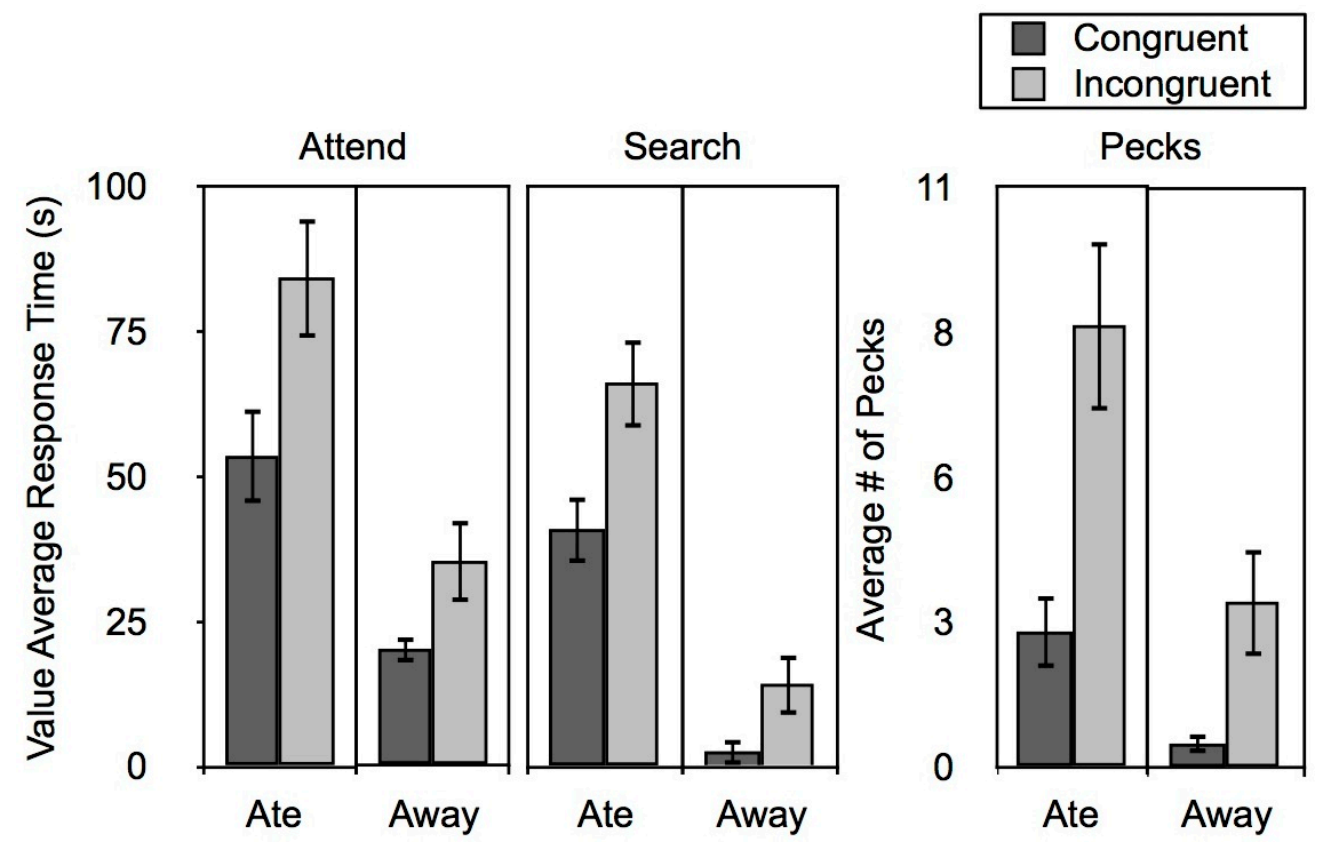

Figure 13. Overall response by prey destination - Average response in search and attendance time and number of pecks.

A One-Way ANOVA was used to compare means between conditions: prey being eaten $(N=50)$, taken away $(N=59)$, and instances where both occurred $(N=7)$. Trials where there was no prey found were not included. On average, all three response dimensions were all higher when prey was consumed (Attend $=72.60 \mathrm{~s}$, Search $=$ 56.66s, Pecks $=6.18$ ), than when prey was taken away (Attend $=26.59 \mathrm{~s}$, Search $=$ $21.37 \mathrm{~s}$, Pecks $=1.61$ ) or both consumption and removal occurred (Attend $=41.86 \mathrm{~s}$, 
Search $=23.29 \mathrm{~s}$, Pecks $=0.00)$. When examining prey destination across all congruent and incongruent trials in both experiments, differences in all three dimensions appeared significant: attendance duration $(F(2,113)=17.198, p=.000)$, search duration $(F(2,113)=20.589, p=.000)$, pecking frequency $(F(2,113)=$ 9.795, $p=.000)$.

\section{Discussion}

The results of Experiment 1, which displayed trials of 'possible' or 'impossible' or mathematical outcomes, show that robins spent significantly more time searching during incongruent than congruent trials. Robins also spent on average, more time searching after exposure to an incongruent trial than a neutral exposure (of simply the apparatus itself, and no trial or prey), but conversely spent on average less time searching after congruent trials than a neutral exposure. The same pattern of results held true for Experiment 2, where trials were manipulated so that outcomes were categorically 'possible' or 'impossible' rather than mathematically true or false. Looking at the data from both experiments, robins also spent more time searching when consuming the worm than when provisioning or caching the worm.

Rugani et al.(2009) suggests that signature limits might differ depending on whether a species is altricial or precocial, or that perhaps task specificity plays a role. The domestic chicks (Gallus gallus) in his study, for example, could perhaps have signature limits influenced by their brood size (on average 8-10 chicks). Following such logic, if task-specificity related to New Zealand robin ecology plays a role in defining signature limits for number discrimination (see Chapter 2) or addition and subtraction in the present study, robins' signature limit could be closely related to the average 'running inventory' of insects stored in caches at a given time for each individual. Such a limit would not however, be broached in the present data, with prey items totalling no higher than 3 in any condition, but could potentially be an avenue of exploration in future studies.

The inherent curiosity of New Zealand robins (see Chapter 1) not only necessitated control exposures to the apparatus without any trial or prey present, but also saw a great deal of variation amongst individuals. Given the opportunity to continue returning and searching, in many cases an individual robin might return to the apparatus multiple times over the course of several hours to resume 
investigating in some, but not all, cases. While an extended investigation of individual variation in continued searching would provide interesting data for a study focused on hunting and pilfering strategy, this type of lengthier, in-depth investigation into attention and memory related to placement of prey items was not applicable to the present study.

Whether an accumulator model (Meck \& Church, 1983) or other cues such as individual characteristics lend themselves to the ability to add and subtract small numbers of items remains an unanswered question. Attention to individual physical characteristics of same-species or even differing species of prey is an area that has not yet been experimentally approached with New Zealand robins. Infants, for example, rely on number rather than spatial information, when the objects in an array differ individual by feature (shape, colour), whereas the opposite is true for homogenous groups of items (Feigenson \& Carey, 2003). Positive results indicate the possibility that robins, like other species, have access to basic arithmetical information in the absence of language. This finding provides further possible evidence supporting the theory that a basic system of calculation has evolved as part of a suite of features characterising a system representing number. 
CHAPTER 5: Study 4 - Response to Human Gaze 
Attentional cues and gaze direction are important components of communication and the perception of disposition in humans (Baron-Cohen, Wheelwright, \& Jolliffe, 1997) and other social animals (Emery, 2000; Teufel et al., 2009). A variety of species including some primates (Bräuer, Call, \& Tomasello, 2005; Hare, Call, Agnetta, \& Tomasello, 2000; Santos \& Hauser, 1999), canids (Bräuer, Call, \& Tomasello, 2004; Smith \& Litchfield, 2010), horses (Proops \& McComb, 2010) and birds (Peron, Chardard, Nagle, \& Bovet, 2011; Vallortigara \& Regolin, 2006; Von Bayern \& Emery, 2009), follow human attentional cues such as gaze, although the context of response varies widely (competition, cooperation, occluders, subtlety of cues), as does success. Few studies have looked at spontaneous responses of freeranging, wild, or wild-caught animals (Bateman \& Fleming, 2011; Carter et al., 2008; Flombaum \& Santos, 2005; Hampton, 1994). Of the latter, all have investigated gaze from a human-predator aversion context; none have dealt with wild subjects naïve to human or mammalian behaviour.

This endemic New Zealand songbird species, which has evolved in absence of terrestrial mammals until recently, is examined here in order to explore possible evolutionary, ecological and behavioural influences on tracking of gaze direction. North Island Robins (Petroica longipes) face a highly competitive foraging paradigm (Powlesland, 1981), practicing food hoarding (Menzies \& Burns, 2010) and pilfering (Van Horik \& Burns, 2007). Robins may also be sensitive to the presence of conspecifics when caching, frequently re-caching prey (Burns \& Van Horik, 2007), like other food-caching corvids which display cache protection strategies in the presence of observing competitors (Bugnyar \& Heinrich, 2005; Dally, Emery, \& Clayton, 2005). The unique combination of evolutionary isolation, food-hoarding and pilfering behaviour makes this species a highly interesting candidate as a model for investigating questions surrounding basic social intelligence.

This study asks whether wild North Island robins, a species unique to New Zealand, are able to respond to what humans can and cannot see. To do so, six different conditions were presented to 24 adult robins in which they were given the opportunity to 'steal' a mealworm from one of two human competitors. North Island robins are unique in the study of gaze response in that humans and other terrestrial mammals are not natural predators, prey, or direct competitors of this species. Robins are fearless of humans (Menzies \& Burns, 2010), but familiar with predatory 
birds such as falcons. If robins' response to competitive foraging or pilfering opportunities is similar to those of rhesus monkeys (Flombaum \& Santos, 2005), subjects should opportunistically steal from the human experimenter whose gaze is directed away or whose view is occluded from the desired mealworm. In addition to successfully following gaze to make contextually appropriate decision, robins must also be able to spontaneously utilise information about the direction of gaze and visual access in multiple mammalian competitors to make a task-relevant decision.

Robins responded to a competitive food-pilfering task by utilising information about eye visibility of others. Robins were sensitive to human attentional cues but less responsive to eye and profile head direction. The results suggest that in the absence of evolutionary familiarity, robins are still able to understand and respond to humans' social attentional cues, but may do so on the basis of avian eye physiology and visual acuity.

\section{Methods}

Study area and subjects. A total of 24 adult robins participated in this study. Each individual robin was identified by a unique combination of coloured bands around their legs. Some subjects were known to have successfully participated in prior cognitive experiments (Hunt et al., 2008) and have regular exposure to visitors and staff walking through the sanctuary forest paths transecting the territories. Robins are not provided with food outside experimentation, and hunt freely for insects in the leaf litter within their self-established defended territories. North Island Robins (Petroica longipes) face a highly competitive foraging paradigm (Powlesland, 1981) and practice food hoarding (Menzies \& Burns, 2010) and a high rate of pilfering (Van Horik \& Burns, 2007) - as a result, subjects were highly motivated to pilfer prey presented by human experimenters. Each robin was exposed to all 6 conditions, using Latin Squares to alternate the order of exposure, experimenter side (left and right), and experimenter position (visual access or none) across the 24 robins.

Robins, like other endemic birds of New Zealand, have evolved in the absence of terrestrial mammals and are particularly susceptible to predation due to naïve behaviour seen many isolated island populations (Menzies \& Burns, 2010). This naïve response to terrestrial mammals, in combination with food-hoarding and 
pilfering behaviour, makes an ideal wild candidate for comparative cognition research eliciting spontaneous responses. Taking this unique ecological context into account, human experimenters in this study stayed within very close range of 'their' mealworm prey and platform, as robins were unlikely to stay at a great distance while the demonstration portion of the trial was run.

Apparatus and Procedure. Two experimenters performed each experiment, both acting as the competitors (Experimenter One and Two) presenting mealworms (Tenebrio molitar) on square wooden platforms (see Figure 14 below, and Appendix C for links to example videos). Each platform was constructed with identical shallow circular indentations and lined in plastic, to prevent immediate escape of mealworm prey. The female experimenters functioning as competitors were both of similar appearance and build, wore the same clothing (black rain pants, black hooded sweater, brown burlap shoe covers) intended both to function in the field environment and avoid colours that may create unintended signalling (such as white). Experimenter one gave verbal instruction ahead of each trial, and movements were synchronised non-verbally during each trial.

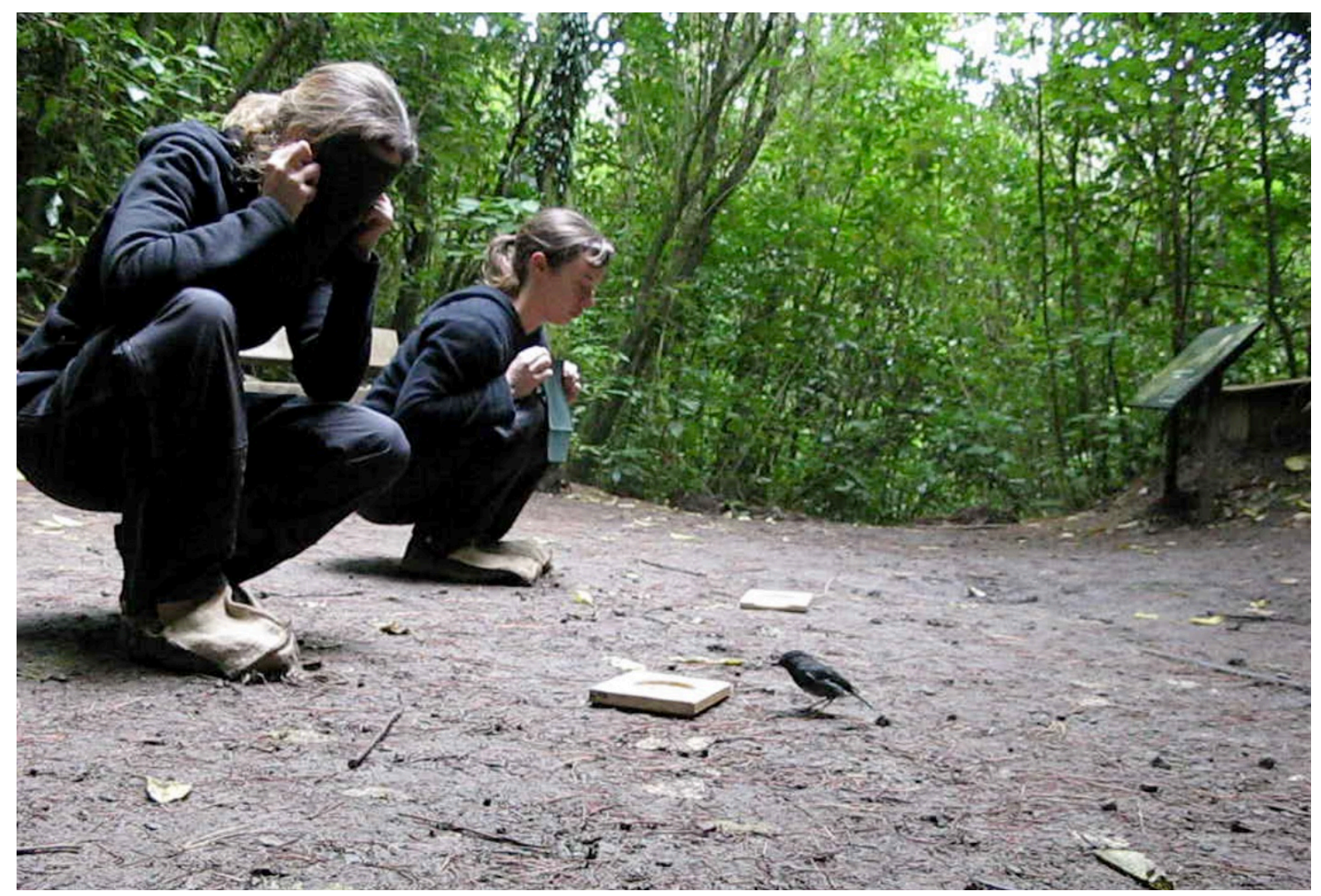

Figure 14. Two experimenters in position during a trial of Condition 5. 
Subjects were located on known established territories, in which the experiment was conducted. Clapping and whistling was used to gain the subject's attention at an appropriate location within the territory. Trials were not run during the interaction of a subject with a mate or fledgling. For video recording, a camera was set up on a flexible field tripod, attached to trees or other landscape features as close as possible to a 90 degree side view of both the subject and experimenters, varying due to the slope of the valley forest landscape and vegetation density.

Each experimenter handled and placed her own platform and mealworm for each trial, to reinforce ownership of these items. Both experimenters approached the subject from the front, each holding a wooden platform and a single mealworm. At approximately $1-2 m$ away from the subject, experimenters squatted and set their platform on the ground approximately $0.3 \mathrm{~m}$ in front themselves. The robin observed from a position approximately equidistant between the two experimenters and their platforms. Continuing to synchronise movements, experimenters then held their mealworms out at waist height (approx. $40 \mathrm{~cm}$ ) over the platform, for 10-20s (visible, pinched between two fingers) until the robin was attending. Experimenters then dropped the mealworms simultaneously onto the platform and immediately assumed the final position described for each condition below. The robin subject was then given the opportunity to approach one of the two competitors, retrieving one of the two mealworms. After retrieval, experimenters both resumed forward position and picked up their respective platforms. In order to avoid aversion or startling due to sound or movement while assuming positions at such close proximity, both experimenters made similar limb and body movements for the same length of time, regardless of the final position. In all conditions, both the initial and final positions were in a squatting position, as robins approach the experimental arena from the ground (and average about $18 \mathrm{~cm}$ in height), and this brings the head and face of the human experimenters more fully into view for the robins.

Conditions. Six different conditions were shown once to each of 24 adult birds; order of exposure was randomised using a Latin Square.

Condition 1. In this condition, subjects were presented with a choice between a human experimenter facing forward (toward mealworm and subject) and an experimenter facing $180^{\circ}$ away (see Figure 15a). The eyes, head and body of the experimenter facing forward were all visible to the robin and positioned facing both 
the mealworm and robin. Conversely, the eyes, head and body of the experimenter facing away were all obscured from view of the participating robin and positioned facing away from both the mealworm and robin. Platforms and experimenters were positioned approximately $1 \mathrm{~m}$ apart, taking into account differences in terrain on each territory.

Condition 2. In Condition 2 (see Figure 15b), subjects were presented with a choice in which the two human experimenters were both positioned between the platforms, and facing the same direction (towards one of the two mealworms). Human experimenters positioned themselves between the two platforms (placed approx. $1.5 \mathrm{~m}$ apart), in relation to the participating robin. Both experimenters initially faced their platform, $180^{\circ}$ away from each other (back to back), and simultaneously dropping the mealworms at the same time onto each platform. One experimenter then turned $180^{\circ}$ to her final position, facing (eyes, head and body) entirely away from the platform she just dropped a mealworm worm onto and towards the back of the second experimenter (and beyond that the second experimenter's mealworm). In the final position, the robin had a clear view of both experimenter's profiles, facing fully toward one of the two mealworms. The experimenter facing $180^{\circ}$ away from her mealworm and platform had a view obscured by experimenter two, and although her eyes, head and body are all positioned facing the second experimenter's mealworm, she could not clearly view either mealworm.

Condition 3. Subjects chose between an experimenter whose eyes and head were facing forward (toward mealworm prey) an experimenter whose eyes and head were facing $90^{\circ}$ to the side (see Figure 15c). Both experimenters' bodies were stationary and facing forward as in Condition 1. After placing their platforms on the ground, experimenters simultaneously dropped the worms from the same height, and one experimenter turned her head and eyes $90^{\circ}$ away from the platform, always in the opposite direction of the second experimenter.

Condition 4. In this condition (see Figure 15d), subjects were presented with a choice between a human experimenter whose eyes were facing forward (toward mealworm prey) and a second experimenter whose eyes were directed $45^{\circ}$ outward (away from other experimenter). Both the face and body of each experimenter were stationary and facing a forward position. Placement of the platforms and dropping mealworms proceeded as described in the Apparatus and Procedure section, 
simultaneously, with one experimenter's eyes turning $45^{\circ}$ away into final position. After experimenter's eyes were in position the subject was given 1 minute to approach and retrieve one of the two mealworms.
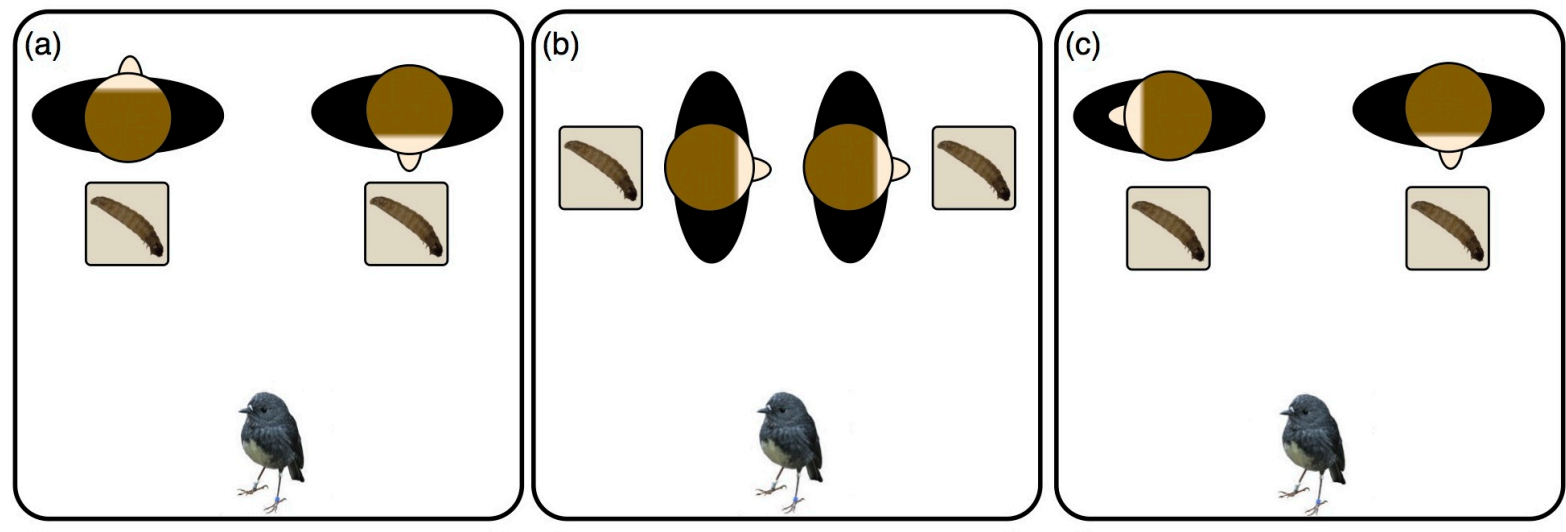

(d)

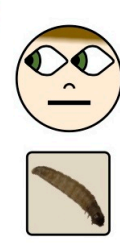

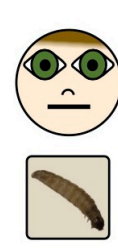

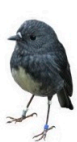

(e)

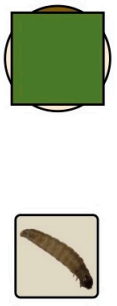

(f)

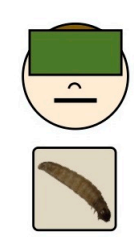

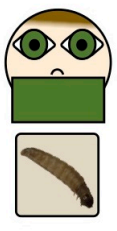
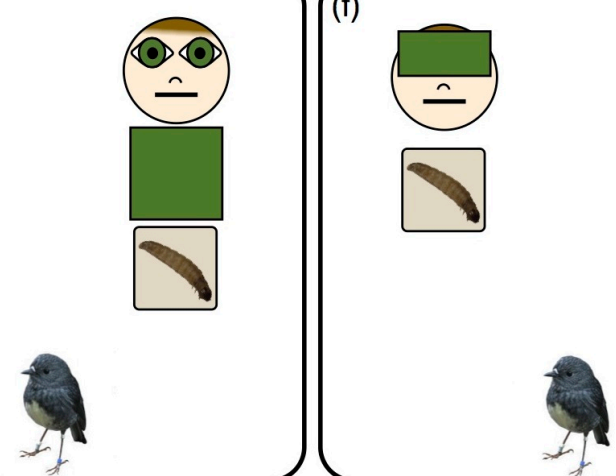

Figure 15. The six conditions presented to robins. In each, one experimenter has full view of the mealworm in front of her, and the view of the other is obscured.

Condition 5. This condition presented subjects with a choice between a human experimenter who was covering their face with a cloth and a second experimenter who covered their chest with a cloth (see Figure 15e). Each opaque, dark green canvas cloth measured $21 \mathrm{~cm}$ wide by $23 \mathrm{~cm}$ in height. After placing platforms in position, both experimenters took the cloths they were holding and simultaneously brought their cloth into position, with one experimenter bringing it in front of her face (completely obscuring the face), and the second holding it in front of her chest, so that in squatting position it appears held below the chin and above her knees.

Condition 6. Subjects were presented with a choice between a human experimenter who was covering their eyes with a cloth and a second experimenter who covered their mouth with a cloth (see Figure 15f). Each opaque, dark green canvas cloth measured $21 \mathrm{~cm}$ wide by $7 \mathrm{~cm}$ in height. After placing platforms in 
position, both experimenters took the cloths they were holding and simultaneously brought their cloth into position, with one experimenter bringing it in front of her eyes (completely obscuring the eyes), and the second holding it in front of her mouth.

\section{Results}

Body orientation. Conditions 1 and 2 presented two human experimenters with different body orientation. In Condition 1, one experimenter faced her entire body (including face and head) toward 'her' platform with 'her' mealworm, and another experimenter faced her entire body away from her own platform and mealworm. Robins selectively approached and retrieved the prey from the experimenter facing away from her mealworm (binomial probability: $p<0.005$; see Figure 16a), suggesting robins approach and pilfer prey based partly on what an individual has visual access to, regardless of species. In Condition 2, experimenters placed their platforms at the side with respect to the observing subject. The robin had to choose between an experimenter facing $90^{\circ}$ to the side and looking at the platform with her mealworm, or an experimenter facing $90^{\circ}$ to the same side, but with her back to her platform and mealworm. Subjects reliably chose the mealworm behind, but not within view of, the experimenter ( $p<0.005$; see Figure 16b), suggesting that while both experimenters only had a profile view of the subject, approach in either condition was not based on generally avoiding the vicinity of the experimenter facing forward.

Head and eye orientation. The next two conditions investigated whether robins were also sensitive to where a human is looking. In the Condition 3, interestingly, robins did not preferentially select between either the experimenter whose head was oriented $90^{\circ}$ to the side or whose head was facing forward ( $p=0.117$; see Figure 16c). Robins did, however, reliably select the experimenter whose eyes were oriented $45^{\circ}$ to the side ( $p<0.05$; see Figure $16 \mathrm{~d}$ ) rather than the experimenter whose eyes were oriented directly at the mealworm in front of her. These findings suggest that while robins are sensitive to the direction that a human is looking to some extent, their decision-making about what a human experimenter can and cannot see takes different information into account than a primate (Flombaum \& Santos, 2005). This result differs markedly from both other mammalian and avian evidence of sensitivity to information about what can and cannot be seen, but 
suggests that avian reasoning may take different physiological information into account (Cook, 2000; Güntürkün, 1999).
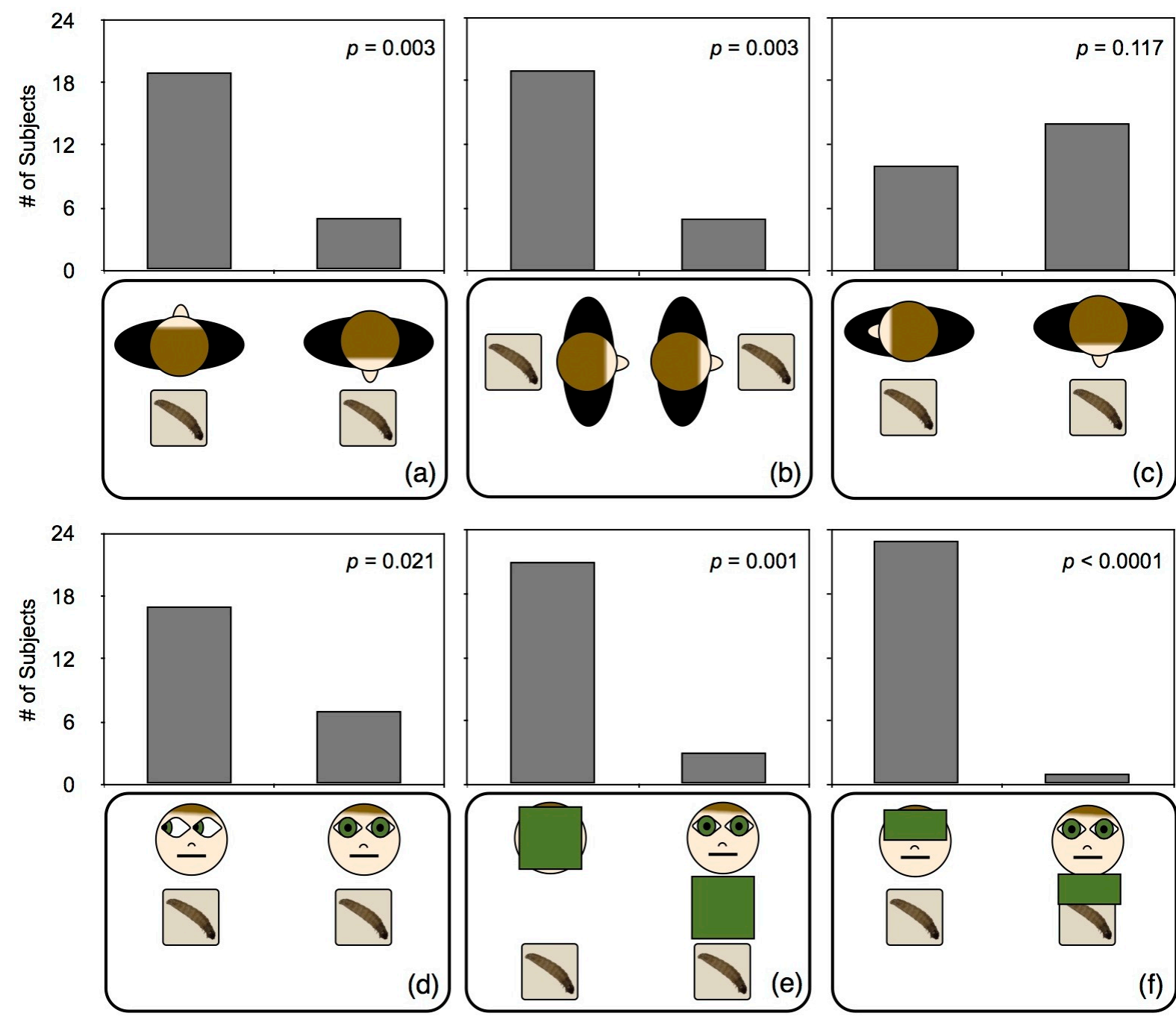

Figure 16. Results for all six conditions, displaying number of subjects pilfering from each experimenter, with one trial per condition per subject.

Visual occlusion. The final two conditions investigated whether robins were able to take into account a physical barrier blocking an experimenter's visual access to her mealworm prey. In Condition 5 , robins preferentially pilfered the mealworm of the experimenter with an opaque cloth barrier held in front of her face ( $p \geq 0.001$; see Figure 16e) rather than the experimenter with a cloth barrier held in front of her chest (see Figure 14). Robins also overwhelmingly selected the mealworm of an experimenter with an opaque cloth barrier held in front of her eyes $(p>0.0001$; see 
Figure 16f) as opposed to an experimenter with an opaque cloth barrier in front of her mouth. Results from both conditions indicate robins are able to take into account whether a visual occluder is obstructing another agent from clearly viewing prey, when making decisions about pilfering or competitive foraging.

\section{Discussion}

North Island robins (Petroica longipes) responded aversively to the presence of human eyes in a choice task to 'steal' mealworm prey in five of six conditions presented. Robins did not distinguish between human experimenters facing towards or $90^{\circ}$ away from prey, and were equally likely to steal the mealworm of either experimenter when these two positions were presented. Robins' preference in selectively choosing to steal from the human experimenter without visual access in every other case appears to indicate sensitivity to the gaze in a species other than itself. Given the relatively aggressive, competitive, and only moderately social nature of this species (very small social networks with comparison to more highly social birds) (Menzies \& Burns, 2008), responding with any sensitivity to eye gaze in an invasive mammalian species is an interesting behaviour to consider, and begs a number of questions about both influencing factors and context. While the present study shows a very basic level of response to the presence of eyes facing desired prey, it leads to the more intriguing question about whether this naive species would successfully and flexibly reason about visual access in a species with the vast differences in visual systems seen in humans with comparison to passerines.

An interesting difference between North Island robins and other species showing these abilities (Flombaum \& Santos, 2005; Kaminski, Riedel, Call, \& Tomasello, 2005; Smith \& Litchfield, 2010), is that robins fail to differentiate human experimenters with heads facing $90^{\circ}$ away from those looking forward, even while succeeding in other conditions, including when only the angle of gaze direction itself, albeit with slightly less success than other conditions.

One potential consideration is whether robins might be making decisions in this context within the framework of an avian visual system, regardless of species of their competitor. Passerine visual systems are functionally very different from human vision in that there is a much wider range of vision peripherally, and in contrast to having acute binocular frontal vision and poorer peripheral vision like humans, they 
have acute monocular vision laterally, and much less acute frontal binocular vision (Güntürkün, 1999). Therefore, in the instance where robins were asked to choose between a human facing frontally and facing $90^{\circ}$ laterally to the object, both at short distances, the subject may possibly fail to preferentially select in this condition based on an underlying response limited to avian vision systems, in which case both experimenters could be attending the contested mealworm. The current array of modern predators has only been exposed to indigenous species for roughly the last 800 years (Whitwell, 2011). (This is not to imply they are naturally predator-free; New Zealand falcons, Falco novaeseelandiae, are a natural predator of robins and other passerines that predate other introduced predators, for example.) In lieu of evolutionary influences or signalling via dichromatic eye morphology, one possibility is that robins are responding to human gaze-direction as though it functions like that of songbirds. Such behaviour may provide support of the 'like me' hypothesis underlying basic social cognition described by Meltzoff (Meltzoff, 2007). Whether lack of co-evolution has played a role in limiting robins' ability to respond to differing visual perimeters in introduced species remains, however, an open question that requires further experimentation to investigate as any possible contributing factor to this behaviour.

To date, however, such experiments have been performed on hand-reared captive birds (Bugnyar, 2011; Peron et al., 2011; Range, Horn, Bugnyar, Gajdon, \& Huber, 2009; Von Bayern \& Emery, 2009; Clayton, Dally \& Emery 2007) or on nonnaïve wild or wild-caught birds, and are exposed exclusively to a predator response situation, where the human acted in a predatory gaze context (Bateman \& Fleming, 2011; Carter et al., 2008; Hampton, 1994), unlike the present study. Jackdaws did appear to respond with increased latency in approach to either one or two human eyes visibly oriented toward food (Von Bayern \& Emery, 2009), differentiating it from other avian studies (Carter et al., 2008; Jones, 1980) where a more basic mechanism appears to be operating more in line with the robins' gaze-aversion to the appearance of two eyes. Von Bayern and Emery suggest that for jackdaws, this could be related to sensing and responding to predation, and point out that jackdaw eye morphology (light iris and dark pupil) makes eye orientation discernible much as humans' do (Von Bayern \& Emery, 2009). Neither of these explanations, however, apply easily in the case of New Zealand robins, as they are unlikely to respond to 
humans as predators, and have eye morphology without any contrast in colour (uniformly dark).

Little is known about whether or how eyes or eye direction plays a significant role in communication between robins, although both inter- and intraspecific signalling occurs via flashing a white frontal spot above the beak (Flack, 1976). A high rate of cache pilferage between mates (Burns, 2009; Burns \& Van Horik, 2007; Van Horik \& Burns, 2007) certainly presents the distinct possibility of caching strategy involving an awareness and response to their conspecifics' eye gaze and direction, like it does with other species dependent on caching behaviour for food sources (Bugnyar \& Heinrich, 2005; Bugnyar, 2011; Dally, Clayton, \& Emery, 2006; Dally et al., 2005; Emery et al., 2004). Such pressures do not however, necessarily predicate an advanced knowledge state about others, and such strategies remain to be investigated in more detail in this particular species.

Another important consideration is exposure to humans. The population in this experiment inhabits a northeastern section of Zealandia (see Figure 2 in Chapter 1, and Appendix B), a wildlife sanctuary open to public visitors. This particular section of forest has a fairly dense network of walking trails that are very accessible and close to the entrance of the park, and therefore see substantially heavier human traffic than the southern reaches of the sanctuary for example. Whether populations of robins in areas less exposed to humans - less transited reaches of the sanctuary an isolated offshore island - would also respond to human eyes or gaze direction in the same manner is an important question. It is entirely possible that the responses of this group of robins could be wholly or partially explained due to regular exposure to both visitor's presence and interactions with experimenters, and that a whole new suite of responses might arise from populations with differing levels of exposure. The loss of an anti-predatory response to mammalian predators within a generation speaks to the plasticity of their behaviour at least as much as it does their evolutionary history and naiveté. While anti-predatory behaviour was developed in some (much reduced) mainland populations after the arrival of western immigrants, it appears to be lost within a generation of being translocated to areas heavily protected from invasive threats (Whitwell et al., 2011). An aversion to the presence of eyes (Jones, 1980), cross-species, simulated, or conspecific, is a naturally occurring 
and almost ubiquitous response in predated species. It is unique that in this case, it also appears to be present in a species not predated by mammals.

The robins' response in the conditions manipulating only eyes or using visual occluders suggest that they are not simply using a behavioural rule to avoid the human experimenter facing forward, as in this case robins would not have selectively chosen between the two experimenters with different occluders. Robins do face aggressive intraspecific competition and pilfering from conspecifics (Menzies \& Burns, 2010). However, it appears unlikely that robins were responding based solely on whether the overall demeanour is threatening for two reasons.

First and foremost, due to their lack of fear of humans or other terrestrial mammals (particularly in this protected population), robins are unlikely to respond to a threatening demeanour even in those mammals that are natural predators of birds such as cats, rats and stoats (Blackwell, 2005; Whitwell et al., 2011). This is further evinced by the experimental procedure itself; after each trial, it was necessary for the experimenters to immediately remove the platforms and remaining worm, or robins would have easily stolen both worms with very little hesitation in almost every case. Preference for pilfering the worm from the experimenter with an obscured visual field did not at all preclude a propensity for pilfering from both experimenters given the opportunity; it simply revealed a strategic priority.

Secondly, robins will often follow walking humans to hunt for insects in the leaf litter that overturns underfoot, and observe and interact with humans at very close range (Menzies \& Burns, 2010). Ecologists have actually gone so far as to speculate that humans play a role similar to that of a Moa from a robin ecology perspective (Atkinson \& Millener, 1991). Such behavioural, ecological and historical contextual details underscore the likelihood that robins are not exclusively responding to predatory threat or posture in humans, as might be the case in other species where such a task is presented. Again, this puts New Zealand robins and other indigenous fauna in a unique position for continuation of such research from a non-predator response perspective that might serve as an informative contrast to other avian gaze studies presently undertaken.

Jackdaws (Von Bayern \& Emery, 2009) and rhesus monkeys (Flombaum \& Santos, 2005) both respond to the presence of human eyes in most conditions, and it appears that robins are also sensitive to human eyes in many conditions. It is 
difficult to directly compare with other studies examining birds' responses to human gaze, because such studies focus on species that have co-evolved with mammals, or experienced hand-rearing and sometimes training (Bugnyar, 2011; Peron et al., 2011; Townsend et al., 2009; Von Bayern \& Emery, 2009). To date, none of these studies have examined a non-naïve species in the wild using a similar competitive object-choice paradigm. Future research is necessary to examine what role coevolution, intra and interspecific social cues, visual field and visual access, exposure to predatory and non-predatory invasive species, age, sex, pilfering strategy, and other ecological contexts might play in shaping a cross-species sensitivity to eyes or gaze, or anything approaching a knowledge of attentional and mental states. 
CHAPTER 6: Study 5 - Response to Human Physical Capability 
The ability to reason about agency and action (Spelke \& Kinzler, 2007) has been explored in a myriad of ways and species. Species from newly hatched chicks to chimpanzees are observed as sensitive to what their predators or competitors can and cannot see (Agrillo et al., 2004; Flombaum \& Santos, 2005; Hare et al., 2001), goal-directed action (Behne, Carpenter, Call, \& Tomasello, 2005; Call, Hare, Carpenter, \& Tomasello, 2004; Peron, Rat-Fischer, Nagle, \& Bovet, 2010; Phillips, Barnes, Mahajan, Yamaguchi, \& Santos, 2009) and gaze direction (Bateman \& Fleming, 2011; Bräuer et al., 2005; Bugnyar, Stöwe, \& Heinrich, 2004; Carter et al., 2008; Jaime, Lopez, \& Lickliter, 2009) and even physical causality (Call, Hare, Carpenter, \& Tomasello, 2004; Horner \& Whiten, 2005).

The role of the limbs in the execution of specific actions is linked to physical causality and has been studied in chimpanzees (Vonk \& Subiaul, 2009), but not yet in avians. For both primates and avians, this may be an important capacity for reasoning about the actions of conspecifics and actions; affecting success in competing for mates, foraging, defence of food stores and possibly assessing strength and weakness in competitors, predator and prey alike. For North Island robins in particular, it begs the question of whether there exists a basic understanding of physical causality as it relates to limb mobility in humans; a species not only much more taxonomically distant than humans and other primates, but one with which exposure on an evolutionary scale is only extremely recent. To this end, this experiment was adapted to test wild robins' abilities to track salient observable cues - visibility of arms and legs - to potentially predict basic 'capability' (the ability to perform specific tasks) in humans.

The majority of studies looking at perception of capability in a human experimenter by another species examine the problem in terms of mental state, looking at intentionality or goal directedness of human experimenters. While chimpanzees did not preferentially distinguish human experimenters who accidentally or deliberately failed to offer food (Povinelli, Perilloux, Reaux, \& Bierschwale, 1998), both chimpanzees and human infants discriminated between 'unwilling' and 'unable' experimenters (Behne et al., 2005; Call et al., 2004), as did capuchin monkeys (Phillips et al., 2009). A recent study suggests that humans and chimpanzees attend differently to goal-directed actions (Myowa-Yamakoshi, Scola, \& Hirata, 2012). Outside of primates, jackdaws (Von Bayern \& Emery, 2009), dogs 
(Hare \& Tomasello, 1999; Miklósi, Polgárdi, Topál, \& Csányi, 1998), dingos (Smith \& Litchfield, 2010), dolphins (Pack \& Herman, 2004), goats (Kaminski et al., 2005) and horses (Maros, Gácsi, \& Miklósi, 2008) all appear to respond in different contexts with varying levels of success to human social cues and gestures such as pointing. A recent study with African grey parrots (Psittacus erithacus)(Peron et al., 2011) found that parrots displayed more requesting behaviour when an experimenter was unwilling to give them seeds - and that their behaviour pattern changed according to attention. However, African grey parrots were unable to use pointing gestures or gaze consistently as cues (Giret, Miklósi, \& Kreutzer, 2009). Jackdaws, in contrast, are able to use pointing as a cue, but only by a familiar caretaker (Von Bayern \& Emery, 2009). Despite such evidence, little has been done to investigate the more obvious and preliminary question of whether other species respond simply to salient observable cues - such as visibility of limbs - to predict a human experimenter's physical capability, regardless of motivational state. As Vonk and Subiaul (2009) point out, such a task does not require the subject to reason about unobservable mental states, or make the inferences necessary to understand a complex gesture such as pointing or subtle mental state differences like 'unwilling'. The present study focuses on presenting experimenters who appear physically rather than motivationally incapable.

In this study, we asked whether wild North Island robins, a species unique to New Zealand, were able to reason about physical capability in humans. To do so, ten different conditions were presented to 25 adult robins in two experiments, in which they were given the opportunity to 'steal' a mealworm from one of two human competitors. If robins' behaviour in competitive foraging or pilfering opportunities takes human capability into account, subjects should opportunistically steal from the human experimenter whose body or limbs are more occluded. In addition to successfully responding to body and limb visibility to make contextually appropriate decision, robins must also be able to spontaneously utilise information about distance, movement and physical access in multiple mammalian competitors to make a task-relevant decision.

The methodology adopted for this study is very similar to that in Vonk's (2009) chimpanzee study on capability, but was adapted to both for the use of wild birds as subjects, and conducting trials out in the field. In addition to either displaying or 
obscuring arms and legs, the appearance of nose/mouth area of the face is also altered. Use of the beak is frequent in territorial defence, pilfering, and the majority of other interaction with and manipulation of the environment around them; for a bird it is as much as a "limb" as other appendages; jackdaws for example, use beak pointing to direct each other's attention to objects (Von Bayern \& Emery, 2009). Most importantly, this choice task is framed within a competitive paradigm. Instead of being asked to choose a food item from a human who will cooperate by providing that item at request when physically possible, robins are asked to choose between stealing the food item from an experimenter, given differing observable physical cues. An additional significant feature is the static location of the mealworm bait in reference to the experimenters. The present study does not attempt to address robins' ability to flexibly apply an awareness of causality or capability, in that the position of the mealworm was not altered in order to appear within reach of only one limb region or another between conditions. It does, however, ask whether a basic understanding of human limb capability in cache defence is present in a species that has not co-evolved with humans.

This study intends to examine whether robins respond to a competitive food-

pilfering task by utilising information of selective visibility of others. If robins respond with sensitivity to limb visibility, it might suggest a basic, rudimentary perception of human capability and causality.

\section{Methods}

Study area and subjects. Wild North Island robins (Petroica longipes) were the focus of this study. A total of 25 adult robins participated in this study. Each individual robin was identified by a unique combination of coloured bands around their legs. Some subjects were known to have successfully participated in prior cognitive experiments (see Appendix A) and have regular exposure to visitors and staff walking through the sanctuary forest paths transecting the territories. Robins are not provided with food outside experimentation, and hunt freely for insects in the leaf litter within their self-established defended territories. North Island Robins (Petroica longipes) face a highly competitive foraging paradigm (Powlesland, 1981) and practice food hoarding (Menzies \& Burns, 2010) and a high rate of pilfering (Van 
Horik \& Burns, 2007) - as a result, subjects were highly motivated to pilfer prey presented by human experimenters.

Procedure. Two experimenters performed each experiment, both acting as the competitors (Experimenter One and Two) presenting mealworms (Tenebrio molitar) on square wooden platforms (see Appendix C for links to example videos). Each platform was constructed with identical shallow circular indentations and lined in plastic, to prevent immediate escape of mealworm prey. The female experimenters were both of similar appearance and build, wore the same clothing (black rain pants, long-sleeved green shirt, black shoe covers) intended both to function in the field environment and avoid colours that may create unintended signalling (such as white). One experimenter gave verbal instruction ahead of each trial, and movements were synchronised during each trial.

Subjects were located on known established territories, in which each set of trials was conducted. Clapping and whistling was used to gain the subject's attention at an appropriate location within the territory. For video recording, a camera was set up on a flexible field tripod, attached to trees or other landscape features as close as possible to a 90 degree side view of both the subject and experimenters, varying due to the slope of the valley forest landscape and vegetation density.

Both experimenters approached the subject from the front, each holding a wooden platform and a single mealworm. Each platform was within easy reach of only the experimenter directly behind it. At approximately $1-2 m$ away from the subject, experimenters squatted and set their platform on the ground approximately $0.3 \mathrm{~m}$ in front themselves. The robin observed from a position approximately equidistant between the two experimenters and their platforms. Continuing to synchronise movements, experimenters then held their mealworms out at waist height (approx. 40cm) over the platform, for 10-20s (visible, pinched between two fingers) until the robin was attending. Experimenters then dropped the mealworms simultaneously onto the platform and immediately assumed the final position described for each condition below. The robin subject was then given the opportunity to approach one of the two competitors, retrieving one of the two mealworms. After retrieval, experimenters both resumed forward position and picked up their respective platforms. 
In order to avoid aversion or startling due to sound or movement while assuming positions at such close proximity, both experimenters made similar limb and body movements for the same length of time, regardless of the final position.

Experiment 1. Twenty-four adult robins were exposed to each of 6 conditions, using Latin Squares to alternate the order of exposure, experimenter side (left and right), and experimenter position (visual access or none) across the 24 robins. Opaque brown cloth was used measuring $210 \mathrm{~cm}$ by $154 \mathrm{~cm}$ to obscure the entire body or limbs of either experimenter. The same type of cloth was used for obscuring part of the face, measuring $26 \mathrm{~cm}$ by $75 \mathrm{~cm}$.

Condition 1. (See Figure 17a) Neither experimenter was covered in this condition. Subjects were presented with a choice between Experimenter One in squatting position, and Experimenter Two in standing position. Arms, legs and faces were all fully visible in this condition.

Condition 2. (See Figure 17b) Both experimenters were covered in this condition, revealing only their head from just above the bridge of the nose. Experimenter One squatted, covered in opaque brown cloth, while Experimenter Two stood, covered in identical opaque brown cloth.

Condition 3. (See Figure 17c) Experimenter One was covered in opaque brown cloth, with her entire body visually occluded from the bridge of the nose to the ground. Experimenter Two was not covered, but draped an identical brown cloth over her lap, without occluding any limbs or a significant portion of her body. Both experimenters squatted in the final position.

Condition 4. (See Figure 17d) Experimenter One covered her legs with an opaque brown cloth, visually occluding her legs from the waist down. Experimenter Two covered her arms with an identical brown cloth, occluding her arms, torso and shoulders. Both experimenters squatted in the final position.

Condition 5. (See Figure 17e) Experimenter One covered her arms with an opaque brown cloth, visually occluding her arms, torso and shoulders. Experimenter Two covered her mouth and nose with a smaller brown cloth, draping an identical brown cloth over her lap, without occluding limbs or any significant portion of her body. Both experimenters squatted in the final position.

Condition 6. (See Figure 17f) Experimenter One covered her legs with an opaque brown cloth, visually occluding her legs from the waist down. Experimenter 
Two covered her mouth and nose with a smaller brown cloth, draping an identical brown cloth over her lap, without occluding limbs or any significant portion of her body. Both experimenters squatted in the final position.
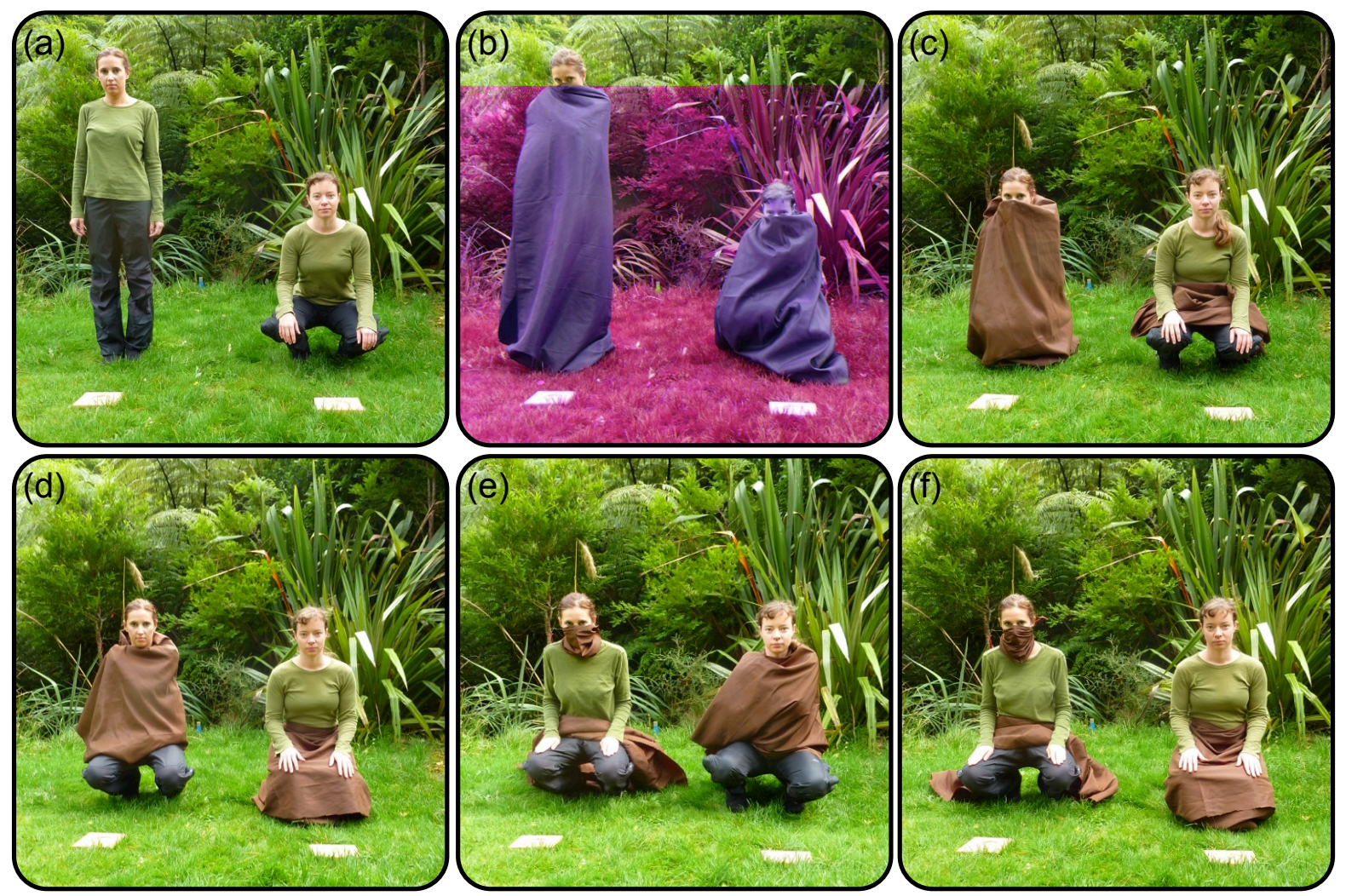

Figure 17. The six conditions presented to robins in Experiment 1, using opaque brown cloth to obscure the body or limbs of the experimenters.

Experiment 2. Twenty-four adult robins were exposed each of 4 conditions, using Latin Squares to alternate the order of exposure, experimenter side (left and right), and experimenter position (visual access or none) across the 24 robins.

Condition 1. (See Figure 18a) Experimenter One squatted behind a wooden plank, her body entirely visually occluded, with her face visible only from just below her eyes (above the bridge of the nose) to the top of her head. Experimenter Two was entirely visible, squatting next to the plank without occluding significant portions of any of her limbs, torso or face.

Condition 2. (See Figure 18b) Experimenter One squatted behind a wooden plank, her body entirely visually occluded, with her face visible only from just below her eyes (above the bridge of the nose) to the top of her head. Experimenter Two 
squatted behind a wooden plank, her body entirely visually occluded, with her face, including eyes and mouth, entirely visible along the outside edge of the plank.

Condition 3. (See Figure 18c) Experimenter One squatted behind a wooden plank, her body entirely visually occluded, with her face visible only from just below her eyes (above the bridge of the nose) to the top of her head. Experimenter Two squatted behind a wooden plank, with her body occluded and arms visible along the outside edges of the plank, and her face visible only from just below the eyes to the top of her head.

Condition 4. (See Figure 18d) Experimenter One squatted behind a wooden plank, her body entirely visually occluded, with her face visible only from just below her eyes (above the bridge of the nose) to the top of her head. Experimenter Two squatted behind a wooden plank, with her body occluded and legs visible below the long edge of the plank, and her face visible only from just below the eyes to the top of her head.
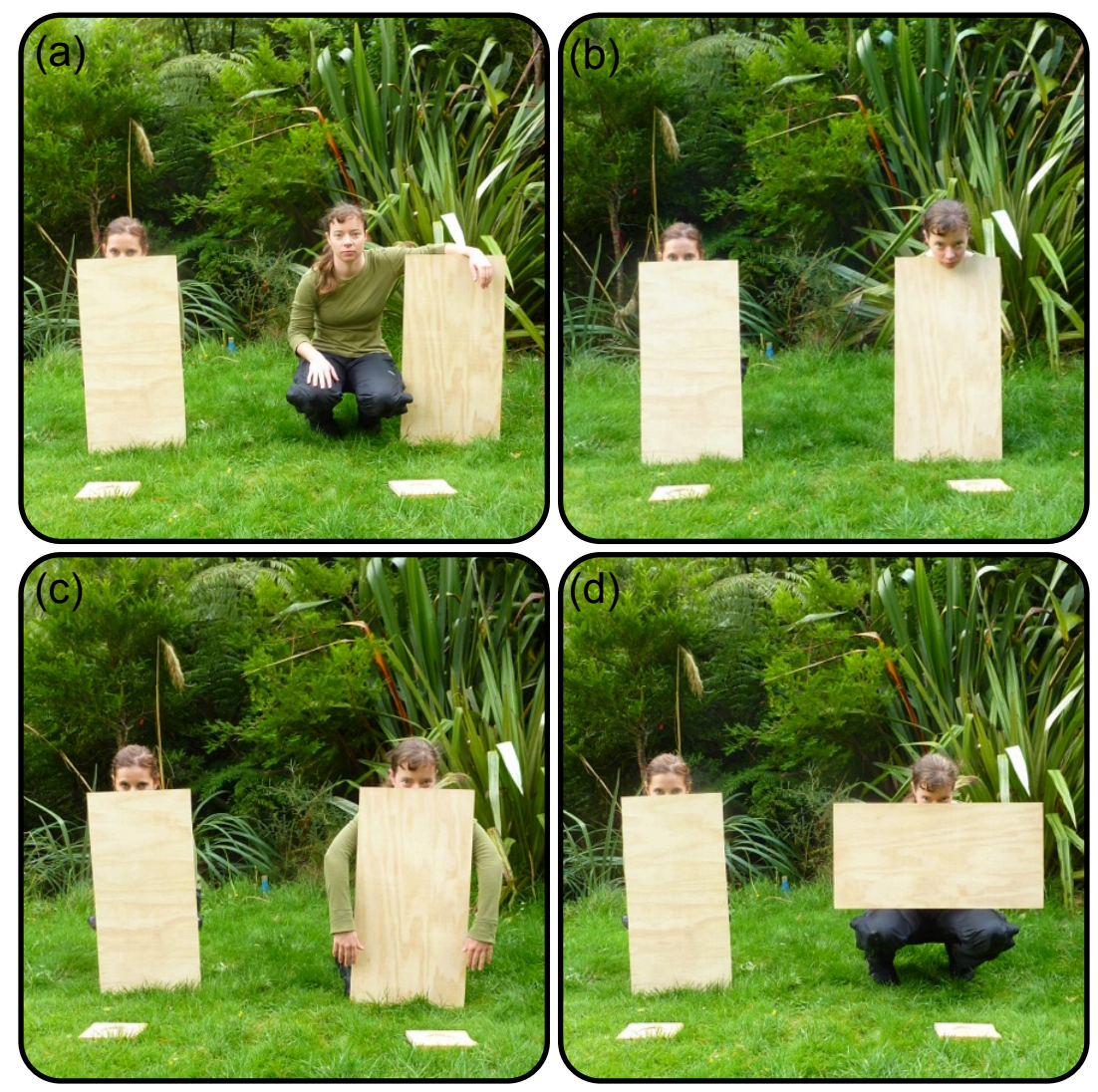

Figure 18. The six conditions presented to robins in Experiment 2, using a wooden plank to obscure the body or limbs of the experimenters. 


\section{Results}

In Experiment 1, for Condition 1 (Figure 17a), there was very little change in latency (1.55s) of response between robins who chose to steal from the visible standing or visible squatting experimenter. The same was true for Condition 4 (Figure 17d) arms covered vs. legs covered (1.77), Condition 5 (Figure 17e) arms covered vs. nose/mouth covered (0.34s), and Condition 6 (Figure 17f) legs covered vs. nose/mouth covered (2.10s). In comparison, there were larger differences in average latency for Condition 2 (Figure 17b) covered squatting vs. covered standing (9.81s), or Condition 3 (Figure 17c) visible squatting vs. covered squatting (8.68s). In Experiment 2, there were noticeable differences in latency to respond between pilfering choice (which experimenter was stolen from) in three of four conditions; Condition 1 (Figure 18a) visible vs. covered (3.81s), Condition 2 (Figure 18b) nose/ mouth visible vs. all covered (8.54s), and Condition 3 (Figure 18c) arms visible vs. all covered (4.95s). In the Condition (d) legs visible vs. all covered difference in average latency of response (1.17s) was small.

Experiment 1. For Experiment 1, Conditions 1, 2 and 3 presented two human experimenters with differing positions and visibility of the entire body. In Condition 1, Experimenter One was uncovered and standing, and Experimenter Two was uncovered and squatting. Robins reliably approached and retrieved the mealworm from the experimenter standing and uncovered (binomial probability: $p<0.01$; see Figure 19a). In Condition 2, were similarly positioned, squatting and standing, but both entirely covered in opaque brown cloth, except the top of the head to just below the eyes. Limbs, nose and mouth of both experimenters were all occluded. Subjects selectively chose the mealworm in front of the experimenter who was standing and covered ( $p<0.05$; see Figure 19b). Results from both of these conditions suggest that proximity to the face is a strong factor in decision-making regarding competitive foraging or pilfering and risk-taking (see Study 4). In Condition 3, Experimenter One was covered and squatting and Experimenter Two was uncovered and squatting. Robins selectively approached and retrieved the mealworm from the experimenter covered and squatting (binomial probability: $p<0.05$; see Figure 19c). Overall, the results of these three conditions indicate that robins preferentially pilfer prey from human experimenters that are standing, and visually occluded. 

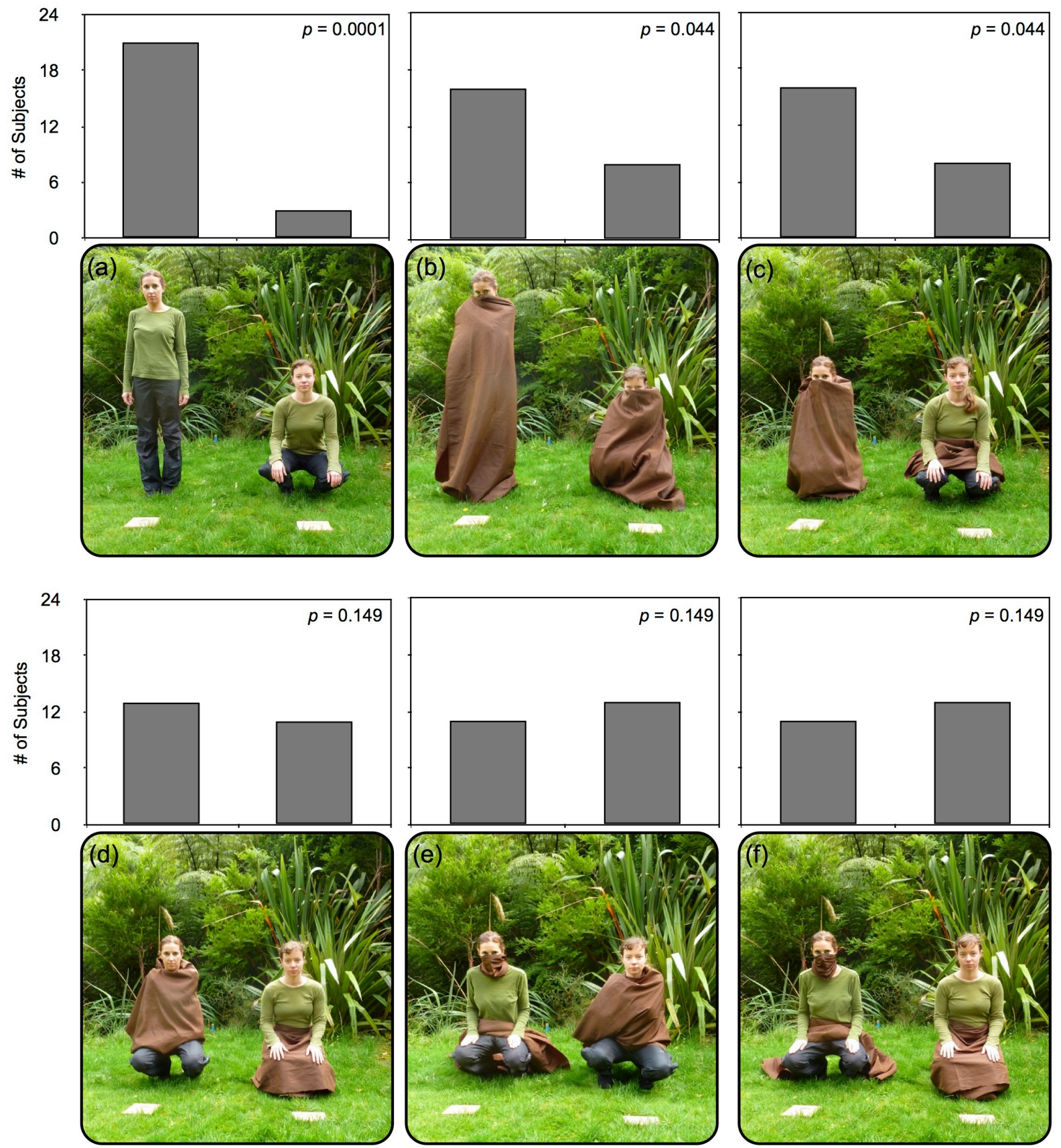

Figure 19. Results for all six conditions in Experiment 1, displaying number of subjects pilfering from each experimenter, with one trial per condition per subject. 
Conditions 4, 5 and 6 presented experimenters in which either legs, arms or nose and mouth were visually occluded. Experimenters were in squatting position for all three conditions. In Condition 4, Experimenter One covered her legs with opaque brown cloth and Experimenter Two covered her arms. In Condition 5, Experimenter One covered her nose and mouth and Experimenter Two covered her arms. In Condition 6, Experimenter One covered her nose and mouth and Experimenter Two covered her legs. Results for all three of these conditions found that robins did not selectively choose either experimenter (binomial probability: $p=0.149$; see Figure 19d-f). Taken together, these results indicate that robins do not selectively avoid only a single limb region (legs, arms, or nose and mouth).

Experiment 2. Experiment 2 alters two fundamental features with comparison to Experiment 1: rather than covering a single 'limb region' (both arms, legs or "beak") at a time, only one limb region at a time is revealed for comparison, and wooden planks are used to occlude experimenters rather than cloth. In these Conditions, Experimenter 1 was always entirely occluded. Experimenter 2 was always fully or partially visible: in Condition 1 she was fully visible (Figure 18a), Condition 2 only her face (including mouth and nose) was visible (Figure 18b), in Condition 3 only her arms were visible (Figure 18c), and in Condition 4 only her legs were visible (Figure 18d). Experimenters were squatting in all conditions. Robins reliably chose the fully occluded experimenter in all four conditions (Figure 20), where Conditions 3 and 4 had slightly stronger significance ( $p<0.005$; see Figure $20 c-d)$ than Conditions 1 and 2 ( $p<0.05$; see Figures 20a-b).

These findings suggest that robins are sensitive to the visibility of all three 'limb' regions (legs, arms, nose/mouth), taking this information into account when making decisions about pilfering or foraging activities. While these results are not directly comparable to primate performance (Vonk \& Subiaul, 2009) where flexible alternation between bait location was also required for successful performance, they do suggest robins possess rudimentary sensitivity to limb visibility within the framework of cache defence and competition. 

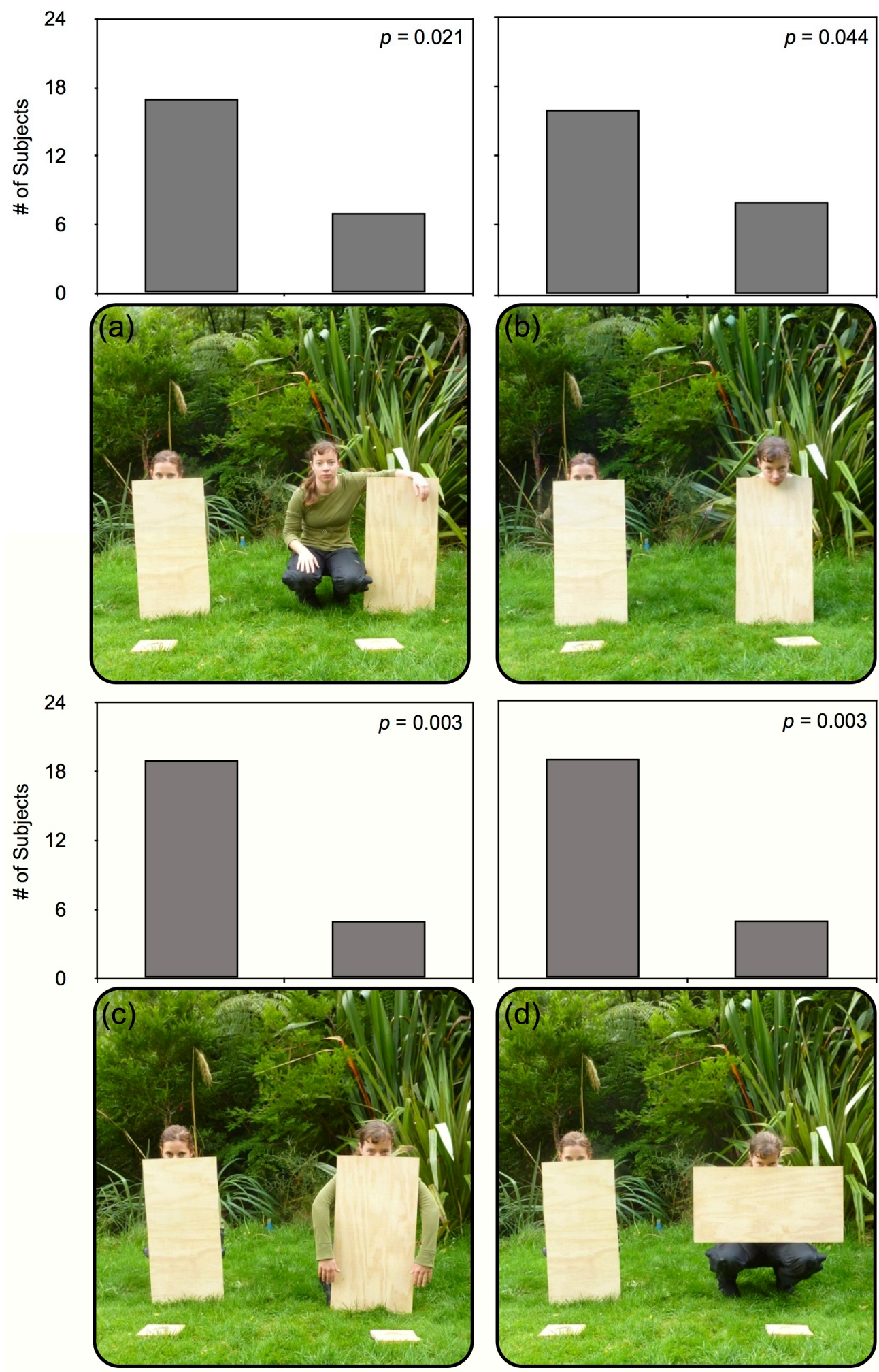

Figure 20. Results for all four conditions in Experiment 2, displaying number of subjects pilfering from each experimenter, with one trial per condition per subject. 


\section{Discussion}

North Island robins demonstrated significantly different responses in pilfering from human experimenters when a limb region is either visible or obscured, but do not appear to differentiate in preference for a specific region. In Experiment 1, subjects preferred to pilfer from an experimenter who was standing or entirely covered in an opaque cloth (Conditions 1, 2 \& 3, see Figure 17a-c), but did not show a preference when only one 'limb region' (arms, legs or nose/mouth) was occluded (Conditions 4, $5 \& 6$, see Figure $17 d$-f). It is possible that the weaker positive p-value for Condition 2 is at least partially due to an effect of avoidance, because of wind fluttering the cloth wrapping the standing experimenter; an unavoidable occurrence in some trials in a peninsular region prone to strong winds such as Wellington. In Experiment 2, subjects were consistently more likely to pilfer from an experimenter who was occluded, rather than an experimenter with a limb region showing.

A simple rule for avoiding the human agent with proportionally more limb area visible is certainly one possible explanation for the robins' response to some conditions. It does not, however, clearly or consistently apply proportionally in effect, where they should have had a less aversive response in a condition where only the nose/mouth area were exposed (see Figure 20b), presenting far less visible surface area, with comparison to a condition where the entire leg region was exposed (see Figure 20d), presenting far greater visible surface area. There was not a proportionally lower level of avoidance of cases with less body surface area visible than in cases with more, and appear to avoid "beak" and "leg" visible conditions equally. Using the same example, when both experimenters are squatting, robins' responses also do not appear wholly explained by the proximity of the exposed limb region - even while the exposed leg region was at a closer approximate distance to the prey and the robin than the exposed nose/mouth area, percentage of responses did not indicate an influence of this difference in distance.

Conversely, in Experiment 1, where experimenters were standing as well as squatting in two conditions, this difference in distance to the head and upper torso in the standing experimenter with comparison to the squatting experimenter may well have a determining factor in the robins' preference for pilfering from the standing experimenter, whose head and torso were at a greater distance from both the prey and the robin itself. It is important in all of these cases to consider context; even 
adult robins with frequent exposure to humans traversing their territory are unafraid and seek out rather than avoid relatively close proximity to completely unoccluded human bodies. Systematic avoidance of only portions of them therefore, based solely on the proportion showing, would be a surprising behaviour in a species showing little hesitation to approach these same body regions within a meter outside of the context of any experiment obscuring limbs. Such behavioural and ecological contextual details underscore that while it may be a contributory factor, robins are unlikely to be responding to proportional visibility of humans alone.

This study also does not in any way conclusively indicate that robins perceive a human's nose and mouth as analogous to beaks, or consider this body region capable of manipulating the environment in the same way. It does, however, establish that they respond by avoiding this facial region much the same way they do visible legs and arms, even in lieu of observing human behaviour in which the nose/ mouth was used in the manipulation of objects or aggression. The birds that took part in these experiments are permanent residents of a fenced sanctuary open to the public, and walking paths transect their territories, inviting regular exposure to human visitors. It is certainly possible that birds in areas of forest less exposed to human visitation, or populations isolated on offshore islands, might show differences in response to the visibility of limb areas.

As noted in Study 4 (Chapter 5), pertaining to visibility of human eyes, evidence shows that this species has a great deal of plasticity in responding to mammalian predators, depending on exposure or lack thereof, and can lose anti-predatory behaviour within a generation of movement to protected areas (Whitwell et al., 2011). This suggests the possibility that behavioural responses toward other non-predatory mammals, such as humans, could also be strongly mediated by exposure. New fledglings, within 6 months of hatching, are often more likely to approach and remain within an extremely close proximity of human arms, legs, and bags in this more heavily trafficked area of the forest than birds 1 year or older (observed in situ). Ecologically, birds (and other species) frequently adapt their behaviour to the physical world around them, from road noise (Slabbekoorn \& Ripmeester, 2007), to changes in biodiversity (Sol \& Lefebvre, 2000). In this context, it is interesting that the data provided by this study appears to demonstrate that a behavioural adaptation 
of pilfering and foraging responses could be applicable to introduced species (mammals).

Previous experimentation has shown that nonhuman animals, both mammals (Call, 2007; Kundey, De Los Reyes, Taglang, Baruch, \& German, 2010; Pattison, Miller, Rayburn-Reeves, \& Zentall, 2010; Santos, Sulkowski, Spaepen, \& Hauser, 2002) and birds (Bird \& Emery, 2010; Pollok, Prior, \& Güntürkün, 2000; Zucca, Milos, \& Vallortigara, 2007) represent and reason about physical objects. Physical contact in particular is a salient cue in physics problems, and is attended to not only by chimpanzees (Povinelli \& Vonk, 2003) whereas bonobos and rooks had difficulty (Helme et al., 2006). A study with chicks (Gallus gallus) (Chiandetti \& Vallortigara, 2011) found that they showed intuitive reasoning about occluded objects. After imprinting upon a specific object as a social partner upon hatching, chicks were given a choice between a screen leaned at an angle that was consistent with that same object being hidden beneath it, or one leant at such an angle that the object could not be hidden behind it. Chicks consistently chose the screen angled such that their imprinted 'companion' could have been hidden beneath it, indicating a rudimentary framework encompassing physical properties of the objects around them. Exact mechanisms and whether such understandings of folk physics is due to causal relations (Bird \& Emery, 2010) or by trial and error (Povinelli, 2000) either in specific contexts or when reasoning about physical interaction altogether is still largely unclear. Whether such an understanding could be extended to physical properties and limitations as it relates to other individual's bodies is yet a further question yet unexplored in most species (Vonk \& Subiaul, 2009).

The present study demonstrates that a biologically naïve bird has the ability to form rules about a physiologically dissimilar species based on observable features but does not answer whether they are generalizable, or disentangle relevant and irrelevant visual cues (2009). If a mealworm was within reach only of an experimenter's legs, but not arms, for example - would robins adapt their response? Given the vast differences in avian and primate physical structure and use of limbs, such a question is even more complex when asked inter- than intraspecies, and of a naïve bird species in reference to humans. Vonk (2009) raises the question of whether causal reasoning is specific to social reasoning, and whether this might be the reason chimpanzees were unable to generalise the physical causality of limbs for 
humans. That can't entirely explain results of either Vonk's or the present study, but makes it all the more interesting that these birds are able to show even a basic systematic response despite lack of co-evolution or clear ecological roles (e.g. predatory). Whether this is due to an underlying mechanism or exposure to humans walking around the sanctuary paths through robin territories is still an open question, begging further investigation with variably exposed populations.

In wild animals, exposure and environmental conditions may play a significant role in how such cognitive abilities play out - even if some shared representations exist across species, responses between species based on differing physiology (a raptor's acute visual system for example) can still be acquired and adapted to through exposure to those species. For example, New Caledonian crows show a context-dependent use of tools for foraging (Hunt, Rutledge, \& Gray, 2006; Taylor, Elliffe, Hunt, \& Gray, 2010; Taylor et al., 2011; Taylor, Hunt, \& Gray, 2012; Taylor et al., 2009) by manipulating wire to form a hook or choosing the right length or type of tool to fit a specific task. Additionally, one study shows that given the right conditions, wolves outperform dogs recognising distal pointing (Udell, Dorey, \& Wynne, 2008), despite research to the contrary (Hare, Brown, Williamson, \& Tomasello, 2002; Miklósi et al., 2003).

A foundational understanding of physical capability underlying reasoning about object-directed action (Phillips et al., 2009) might not be specific to within the primate order. While certain features may well be, a basic framework of observable physical cues and their relation to an agent's physical capability could be a more broadly applicable capacity. Evidence of ravens (Corvus corax) using referential declarative signals amongst themselves in the wild, offering non-edible items in specific social contexts (Pika \& Bugnyar, 2011), or self-agency in chimps (Kaneko \& Tomonaga, 2011), recognising abstract cursor control without direct physical contact may both be seen as related to such a capacity, though the exact extent remains to be seen. The present study attempts to lay the groundwork for further investigation; whether North Island robins can flexibly apply visual information that may be salient to physical capability (i.e. limb visibility and distance to an object), and to what extent the mobility of the visible part of the body influences response (limb versus torso) is certainly an aim for future exploration. 
CHAPTER 7: Study 6 - Perception of Prey Animacy 
Caching species more often store food that has a relatively long shelf-life, such as nuts or seeds (Sherry, 1985), rather than highly perishable food like flesh from living prey or soft fruits. In this respect, North Island, and New Zealand robins in general, are extremely unique in that the food that they hoard throughout their territories are almost exclusively insects that have been hunted and then stunned or killed (and sometimes partitioned if large) before caching (Menzies \& Burns, 2008; Van Horik \& Burns, 2007). Due to the high perishability of the food stored, robins retrieve stores within 1-3 days of them being made (Powlesland, 1980). In the cognitive experiments conducted in the studies presented here on North Island robins, mealworms (and sometimes waxworms) are used as prey both in choice and Violation of Expectancy (VoE) tasks. A small handful of other studies use living prey such as crickets (Spelke \& Kinzler, 2007) and fruit flies (Uller et al., 2003) or in some cases conspecifics (Agrillo et al., 2007; Agrillo et al., 2008; Dadda et al., 2009; McComb et al., 1994) as the countable 'items' in a numerical discrimination task, but the larger portion of such experiments are done with inanimate objects such as tokens or plant-based food (see Chapter 2).

The unique nature of New Zealand robins' storage of hunted prey rather than completely inanimate food objects creates something of a grey area in cognitive behavioural research such as that presented here. For studies such as those presented in Chapters 2 or 4, for example, focusing on quantities of 'items', the 'objects' presented in both VoE and choice tasks are living mealworms or waxworms. These paradigms are formed on the basis of a theory that assumes that the objects responded to are characterised by, and limited to, properties specific only to inanimate objects; persisting over occlusion and subject to laws of physics such as gravity and motion only as a consequence of being acted on (Spelke, 2000; Spelke \& Kinzler, 2007). However, in order to successfully adapt hunting strategy for different prey, behavioural responses to that prey must of course be mediated by characteristics of that prey: agent rather than an object; causality and self-propelled motion, varying mobility and goal-directedness (whether or not this is perceived as such by either the prey or the predator; such is not assumed here) (Leslie, 1994; Spelke \& Kinzler, 2007). Whether some basic representation of agency is used, or behavioural rules are developed by experience and tailored to differing prey behaviour (e.g. movement for escape) - ostensibly, for robins, prey is first animate, 
and then strategically killed and stored as objects. This study aims to begin investigating the boundary between the animate-inanimate distinction in such prey, and how responses of North Island robins might be influenced by expectations specific to the type of prey, or the animacy state of prey.

Arguably, the most important function of an agency system is agency detection the ability to discriminate between things capable of goal-directed action and selfpropulsion and things that are not; an ability that appears to have its own dedicated neural network (Gobbini et al., 2011). There are obvious costs and benefits resulting from the ability (or failure) of an animal to successfully and reliably detect agency, particularly in reference to predator-prey interaction, where life and reproduction often hangs in the balance not just in detecting animacy in surroundings, but detecting it quickly and identifying threats or opportunities (Barrett, 2005; Barrett \& Behne, 2005). Over the course of time, it is of course exactly this interaction of success and failure in recognition that shapes the evolution of such detection systems present in nearly all species (Barrett \& Behne, 2005). This very basic categorisation of the world - animate/agent and inanimate/object - and the resultant ability to respond differently to each category (Barrett \& Behne, 2005; Spelke \& Kinzler, 2007) allows species to integrate more complex behaviours within such a framework; responding with a fine-tuned suite of behaviours geared toward specific prey, like bee-eaters (Watve et al., 2002), or in the case of human infants, the ability to built entire hierarchies of subcategories with associated representations and contexts.

Development of the animate-inanimate distinction (Gelman \& Spelke, 1981; Gelman \& Opfer, 2002) in humans is not a simple or straightforward process. Wheatly et al. (2007) hypothesise the engagement of the mirror system in understanding animate agents. By the age of 6 months, infants associate some animate properties with people, but they do not associate the appropriate properties to the broad category of animates and inanimate until at least the middle of the 2nd year (Rakison \& Poulin-Dubois, 2001). At preschool age, children do not yet appear to consistently differentiate subcategories of inanimacy, such as dead, inactive, unreal or absent (Carey, 1985; Gelman \& Gottfried, 1996; Slaughter, Jaakkola, \& Carey, 1999). By the age of about 4 however, causal cues to death prevent the attribution of agency to animals and people, whereas cues to sleep do not - and this 
particular pattern of development appears to remain consistent across cultures. By the age of 4 , children understand the concept of death as the cessation of agency in such a way that causal cues relating to death inhibit the attribution of agency to animals and people (Barrett \& Behne, 2005). In animals however, any kind of understanding of their perception about cessation of animacy is considerably more difficult to broach with such specificity.

Causality and self propelled motion are key features of identifying animacy. In a habituation/dishabituation experiment, for example, when exposed to videos either with natural causal sequences (such as a hand picking up an item and carrying it off screen), or unnatural sequences (where the hand does not pick up the item but both move off screen), chimpanzees (Pan spp.) looked significantly longer at unnatural physical events than natural ones (O'Connell \& Dunbar, 2005). Newly hatched domestic chickens (Gallus gallus) also show a sensitivity for self-propelled causal agency, and when shown objects that are either display self-propelled or caused motion (Mascalzoni, Regolin, \& Vallortigara, 2010), prefer to associate with selfpropelled objects as companions.

According to Rakison and Poulin-Dubois (2001), drawing on patterns emergent from infant research on representation and categorisation of animacy, there are seven characteristic properties of animate-inanimate distinction, all of which are related to physical or psychological causality. In this proposal, animates display 1) the self-propelled onset of motion, rather than externally caused, 2) a smooth line of trajectory, as opposed to irregular, 3) causal action at a distance, rather than from physical contact, 4) a contingent rather than non-contingent pattern of interaction, 5) an agent type of causal role rather than recipient, 6) a purposeful, goal-directed action, as opposed to without aim, and 7) the influence of an intentional mental state rather than accidental. In a review of research based on visual interpretation of very simple two-dimensional shapes interacting in either a causal or self propelled way, Scholl and Tremoulet (2000) conclude that the visual system involves a mechanism which focuses on features like causality and animacy in objects. Such a system would inform causal and social structure of surroundings, the same way that the visual system has a mechanism focusing on physical features in order to inform 3dimensional structure of the surrounding world. 
Agency detection and perception of varying states of animacy is perhaps unsurprisingly under-investigated in the context of caching live prey in the wild, given the relative rarity of this behaviour, and inherent difficulties in experimentally determining underlying cognitive aspects of such an adaptive behaviour. A few species depend on either killing, paralysing, or in some cases even maintaining live prey and then caching it for short or long periods of time depend on such distinctions for the success or failure of their livelihood. Jaguars often hang carcasses in trees or sometimes bury them, wolves and foxes sometimes bury whole or partial carcasses, and some species of moles and shrews store earthworms in 'knots' underground (Vander Wall, 1990). The majority of caching birds however, appear to cache less perishable plant-based food such as seeds, although New Caledonian crows are unusually skilled at using tools to poke insect larvae out of trees (Bluff, Weir, Rutz, Wimpenny, \& Kacelnik, 2007; Taylor et al., 2012; Taylor, Hunt, Holzhaider, \& Gray, 2007), and Scrub Jays (Aphelocoma coerulescens) in experimental conditions will only recover caches of highly perishable worms or crickets after a shorter interval, and switch to recovering less perishable peanuts after longer intervals without access (Clayton \& Dickinson, 1999; Clayton, Emery, \& Dickinson, 2006b). Scrub jays also sometimes cache insects or fruit in addition to seeds in the wild (Emery et al., 2004), although the exact extent to which Scrub Jay caches in the wild are composed of insect matter seems less clear (Sherry, 1985). Accurately judging the time of death is important to knowing the perishability of the food source, or being able to distinguish paralysis from death (and hunt accordingly) can lengthen the shelf life of a food.

Prey preference and selection is often based on movement - biological motion (Johansson, 1973). Domestic chicks (Gallus gallus) for example, prefer to peck at insect-like elongated stimuli moving in a direction orthogonal to their longer axis (i.e. 'sideways' rather than 'forward') in a computer simulation test (Clara, Regolin, Vallortigara, \& Rogers, 2009), and even prefer to approach a point-light animation exhibiting biological motion patterns (Vallortigara, Regolin, \& Marconato, 2005). Grey partridge chicks' (Perdix perdix) food preference is influenced by colour (yellow/ green), size (large but able to be swallowed whole), and movement - preferring living to dead insects whether fast or slow moving, as well as being a determining factor in the small insects that were selected (Moreby, Aebischer, \& Southway, 2006). 
This study examines the response of North Island robins to a within-subject study composed of 2 experiments, testing the Violation of Expectancy (VoE) of both the type (and mobility) and state of animacy of prey. In the Experiment 1, three different types of prey are presented, with varying levels of mobility, including mealworms (Tenebrio molitor larvae), earthworms (Eisenia Andrei), and locusts (Locusta migratoria). Each type is presented in one congruent trial, where the same type of insect placed in the box is revealed by the bird, and one incongruent trial, where no insect is found after one is visibly placed inside the box. It is expected that in all three cases, search times for incongruent trials will be longer than responses to congruent trials. The question this design hopes to answer is whether search times in trials where the insect is not found is significantly longer or shorter for one insect with comparison to another. In Experiment 2, only mealworm prey was presented in varying states of animacy, including live, dead, halved, and in some cases a small twig with roughly the same coloration and dimensions of a mealworm. This experiment aims to discern whether robins will search longer when the animacy state of prey alters, and which alteration of state might result in a longer search time, indicating a sensitivity to animacy state of cached prey.

\section{Methods}

Subjects. A total of 11 individual adult robins participated in this study, with each participating in both experiments. Each subject was identified with the unique combination of coloured bands on the bird's legs. All of the robins used in this study had prior experimental history (see Appendix A).

Apparatus. Trials were performed by presenting subjects with either mealworms (Tenebrio molitor larvae), earthworms (Eisenia Andrei), locusts (Locusta migratoria), or a small stick (roughly analogous in size and colour to a mealworm) in a wooden encasement designed for Violation of Expectancy (VoE) testing (see Figure 21 and Appendix $C$ for links to video footage). The box measured $9.5 \mathrm{~cm}$ wide, $4 \mathrm{~cm}$ tall, and $17 \mathrm{~cm}$ long, with a thin, particleboard slider protruding from one side which extends out when pulled and slides into the box when pushed. This slider attaches to an upper compartment comprising a clear Petri dish measuring $6 \mathrm{~cm}$ in diameter mounted on the inside of the box. The side of the Petri dish facing the inside of the box into which it can be pulled is covered in opaque black tape so that only the 
contents of the dish is visible when this sliding compartment is pushed into the box. A hole measuring $6 \mathrm{~cm}$ in diameter on the surface of the box allows the subject to see either the sliding compartment $(1 \mathrm{~cm}$ deep), when it is pushed into the box. The well below is accessible when the sliding compartment is pulled out fully, and lined with the same type and size plastic petri dish. The plastic lining of each well served to prevent the mealworms from being able to climb the rough wooden surface of the encasement. The well was covered with a circular brown leather flap approximately $7 \mathrm{~cm}$ across. Insect prey were placed in the apparatus with stainless steel tweezers, to enable the clearest possible view of the prey.

Procedure. In both experiments, the VoE box was used with both the upper and lower compartments filled, regardless of whether the outcome was expected (items were the same) or unexpected (items differed). In that manner, all robins observed prey being dropped only into the lower compartment, and always were only able to retrieve it from the upper. In such a manner, any difference in response based on a perceived difference in depth of the well, or any other aural or visual cues that may not be apparent to the experimenter, such would be the case both for expected and unexpected events.

Experiment 1: Prey type. Robins were shown 6 different hiding events with order controlled using a Latin Square, of which 3 were congruent (the same type of insect dropped in was found) and 3 were incongruent (no insect was found) (see Table 5).

Table 5. Congruent and Incongruent conditions presented in Experiment 1, altering item type.

\begin{tabular}{llll}
\hline \multicolumn{4}{c}{ Experiment 1 } \\
\hline Prey Item & Condition & Viewed Entering Box & Revealed \\
\hline \multirow{2}{*}{ Locust } & 1 & Living Insect & Living Insect \\
& 2 & Living Insect & Empty \\
\multirow{3}{*}{ Mealworm } & 3 & Living Insect & Living Insect \\
& 4 & Living Insect & Empty \\
& 5 & Living Insect & Living Insect \\
Earthworm & 5 & Living Insect & Empty \\
\hline
\end{tabular}




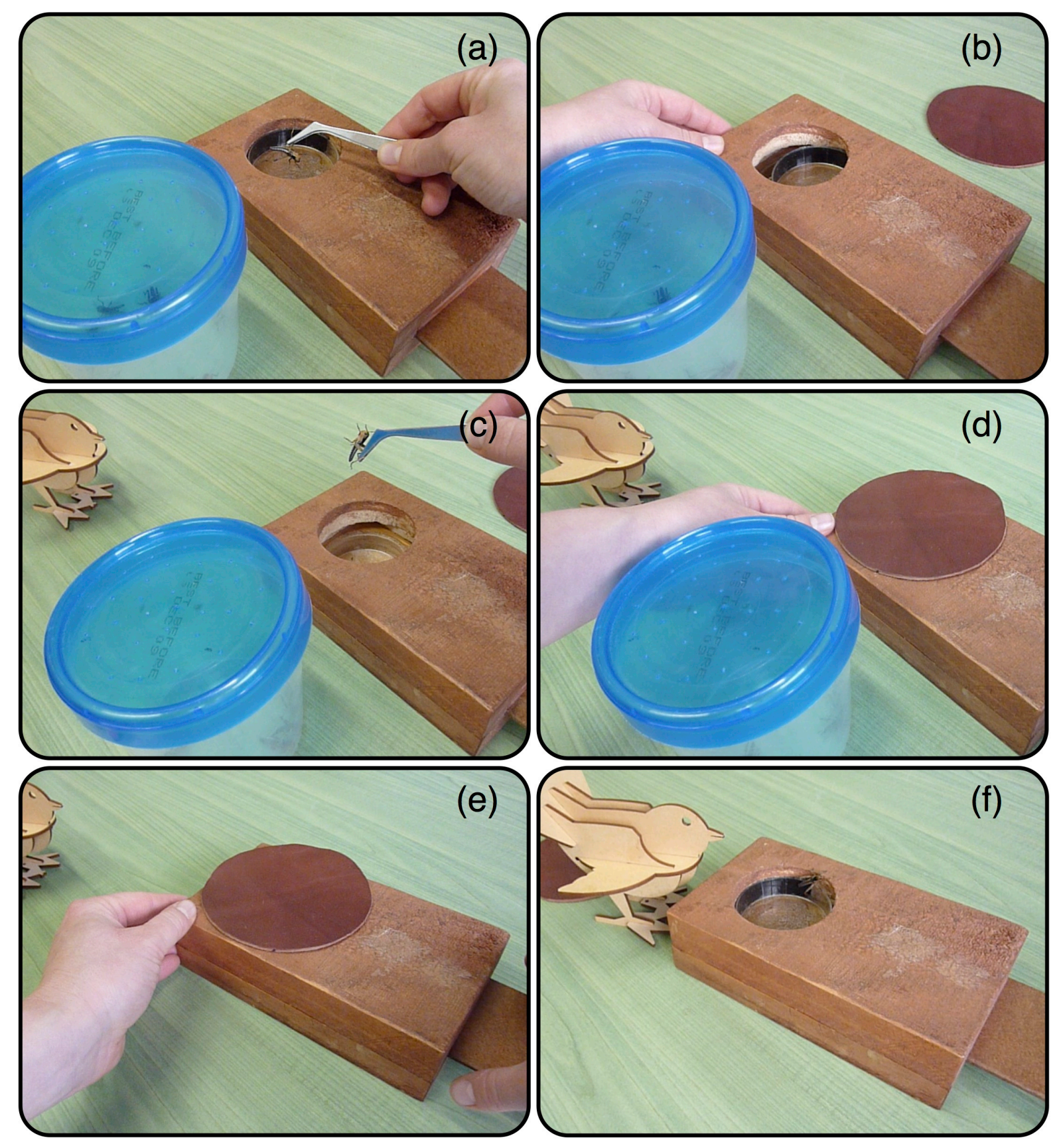

Figure 21. The VoE apparatus: Out of view of the bird, in the upper compartment, (a) insects are placed inside and (b) the compartment is slid out, hiding it. In view of the bird, in the lower compartment (c) insects are placed inside (d) the lid covers the well, (e) the drawer is slid in, and (f) the bird allowed to access the upper compartment.

The sliding compartment of the VoE box was first loaded with an insect (mealworm, earthworm or locust) intended for the robin to find, out of view of the experimental subject, and the sliding compartment was pulled fully out so that the contents was not visible. In the events where nothing is found, no insect was pre- 
loaded into this compartment. The VoE box was then placed so that it was clearly visible to the subject, within a 1-3m distance of the robin's viewing position. A clear plastic container housing a number of the insects about to be dropped into the VoE box is set next to the box, in order that the robin sees the insects actively moving about inside the container. One live insect was taken with tweezers and first displayed clearly for approx. 4s by holding it directly over the well, in order to give the robin the opportunity to clearly see the type of insect and its movement. It was then dropped in clear view of the robin into the empty bottom compartment of the VoE box, and subsequently covered with the leather flap. The hidden sliding compartment drawer is then pushed closed, so the robin finds only what is in the upper compartment (either empty, or with the same type of insect that was seen dropped in). Lastly, robins were given the opportunity to approach and uncover the well, and retrieve a combination of worms either consistent or inconsistent with the category of worms they observed being added or removed.

Experiment 2: Prey animacy. In this experiment, robins were shown 8 different hiding events with mealworm prey hidden and then found in different states (live, dead, halved), or in some cases substituted by a small stick (see Table 6). Of these, 2 were found congruent (e.g. Live-live, dead-dead), 2 were halved (live-half, deadhalf), 2 were found living (dead-live, stick-live), and 2 were found inert (live-dead, live-stick).

Table 6. Congruent and Incongruent conditions presented in Experiment 2, altering item animacy.

\begin{tabular}{lll}
\hline \multicolumn{3}{c}{ Experiment 2 } \\
\hline Condition & Viewed Entering Box & Revealed \\
\hline 1 & Living Mealworm & Living Mealworm \\
2 & Living Mealworm & Dead Mealworm \\
3 & Living Mealworm & Half Mealworm \\
4 & Dead Mealworm & Dead Mealworm \\
5 & Dead Mealworm & Living Mealworm \\
6 & Dead Mealworm & Half Mealworm \\
7 & Living Mealworm & Stick \\
8 & Stick & Living Mealworm \\
\hline
\end{tabular}


Order of exposure was controlled using a Latin Square. The sliding compartment of the VoE box was first loaded with state of insect (or stick) intended for the robin to find, out of view of the experimental subject, and the sliding compartment was pulled fully out so that the contents was not visible. The VoE box was then placed so that it was clearly visible to the subject, within a $1-3 \mathrm{~m}$ distance of the robin's viewing position. A clear plastic container housing a number of the insects about to be dropped into the VoE box is set next to the box, in order that the robin sees the insects either actively moving or inert inside the container. One insect was taken with tweezers and first displayed clearly for approx. 4s by holding it directly over the well, in order to give the robin the opportunity to clearly see the type of insect and its state (active movement or inert). It was then dropped in clear view of the robin into the empty bottom compartment of the VoE box, and subsequently covered with the leather flap. The hidden sliding compartment drawer is then pushed closed, so the robin finds only what is in the upper compartment (either alive or inert). Lastly, robins were given the opportunity to approach and uncover the well, and retrieve a combination of worms either consistent or inconsistent with the category of worms they observed being added or removed.

\section{Results}

A video analysis was performed looking at 3 different dimensions of response behaviour: First, general attendance duration (AD) - the amount of time the subject spent within 1 meter of the apparatus, generally attending even when not actively searching. Second, active search duration (SD) - the total amount of time the robin spent actively examining the apparatus or leather cover. Third, pecking frequency (PF) - the number of times the subject pecked with its beak at any part of the apparatus. The data was also analysed jointly (across both Experiments 1 and 2) looking at the destination of the prey obtained by the robin, and whether it was eaten immediately or removed for caching or provisioning, to examine any differences that might be seen in response duration as a result.

Experiment 1: Prey type. This experiment involved hiding events using three types of prey; mealworms, earthworms and locusts. An experimenter displayed an event where the robin found prey that was either consistent with the prey seen being dropped into the box, or found the box empty. 
Significant differences resulted between the responses in congruent and incongruent trials in multiple dimensions (see Figure 22). A One-Way ANOVA was used to compare means between conditions: congruent trials $(\mathrm{N}=33)$, incongruent trials $(\mathrm{N}=33)$, and neutral exposures $(\mathrm{N}=11)$. Overall, responses for congruent trials were on average lowest values (AD 25.76s, SD 21.88s, PF 2.97) and incongruent trials measured highest (AD 63.79s, SD 53.88s, PF 5.70), while neutral trials on average fell in the middle (AD 33.60s, SD 25.90s, PF 4.35). The difference in means between conditions were overall significant: attendance $(F(2,83)=12.339, p=$. $000)$, search duration $(F(2,83)=14.713, p=.000)$, and pecking frequency $(F(2,83)$ $=4.766, p=.011)$.

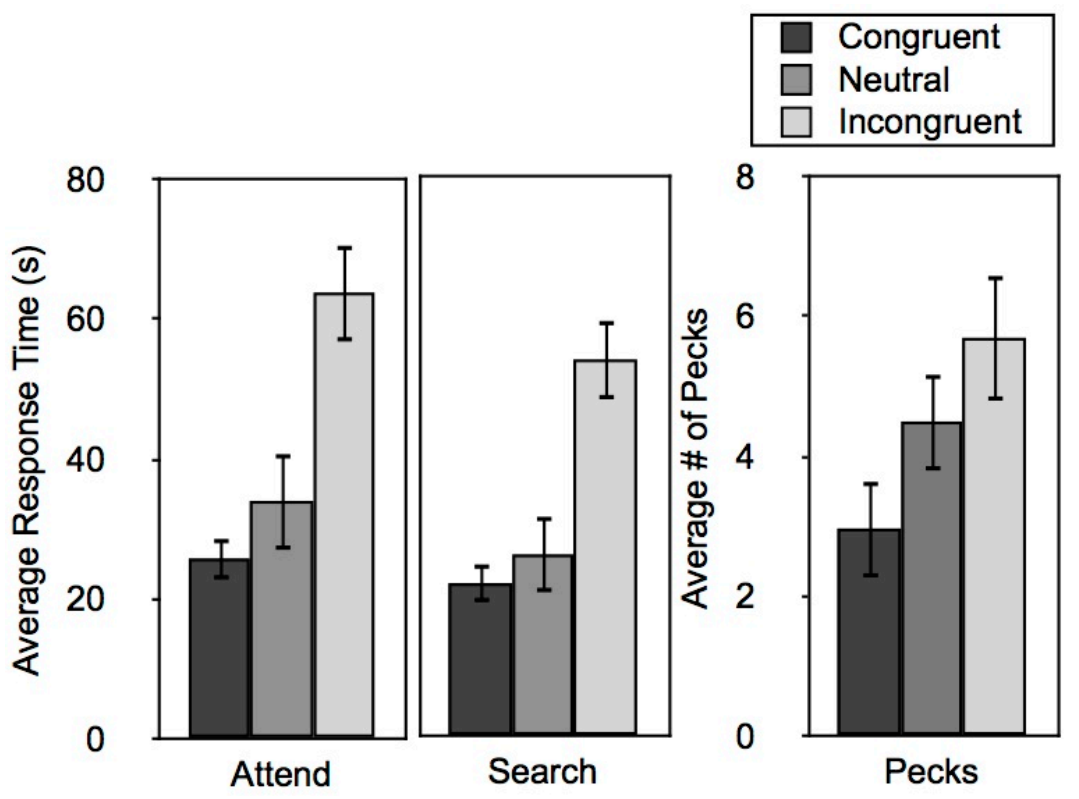

Figure 22. Experiment 1 (Prey type) - Average response in search and attendance time and number of pecks across congruent and incongruent trials.

Independent-Samples T-Tests were used to compare means between each condition (see Figure 22). Congruent and incongruent trials were significantly different in two of three measures: attendance (mean $\Delta 36.33 \mathrm{~s} ; t(53)=-4.021, p=$ 0.000 ) and search (mean $\Delta 31.26 \mathrm{~s} ; t(53)=-4.236, p=0.003$ ), but not pecking (mean $\Delta$ 2.03; $t(53)=-1.635, p=0.205)$, as were neutral and congruent trials: attendance $($ mean $\Delta 7.84 \mathrm{~s} ; t(51)=-1.070, p=0.009)$ and search $($ mean $\Delta 4.02 \mathrm{~s} ; t(51)=-0.734$, $p=0.021$ ), but not pecking (mean $\Delta 0.62 ; t(51)=-3.608, p=0.342$ ). While neutral exposures were on average lower than incongruent trials, they were not significantly so in any of the three measures: attendance (mean $\Delta 28.49 \mathrm{~s} ; t(40)=-2.223, p=$ 
0.213 ) and search (mean $\Delta 13.55 \mathrm{~s} ; t(40)=-2.724, p=0.241)$, and pecking (mean $\Delta$ $2.65 ; t(40)=-1.932, p=0.074)$.

There were also significant differences in response between individual insects for congruent and incongruent conditions (see Figure 23). Paired-Samples T-Tests were used to compare means between conditions. When locusts were used as prey, attendance duration (mean $\Delta 40.73 s ; t(10)=-4.155, p=0.002$ ) and search duration (mean $\Delta 33.73 \mathrm{~s} ; t(10)=-4.409, p=0.001)$, and pecking frequency (mean $\Delta 3.82$; $t(10)=-2.598, p=0.027$ ) were all significantly different between congruent and incongruent trials. For mealworm prey, attendance duration (mean $\Delta 29.82 \mathrm{~s} ; t(10)=$ -2.246, $p=0.048$ ) and search duration (mean $\Delta 25.89 \mathrm{~s} ; t(10)=-2.247, p=0.048$ ) were both significant, but pecking frequency was not (mean $\Delta 1.73 ; t(10)=-1.141, p$ $=0.281$ ). With earthworm prey, attendance duration (mean $\Delta 43.55 \mathrm{~s} ; t(10)=-3.291$, $p=0.008$ ) and search duration (mean $\Delta 37.18 \mathrm{~s} ; t(10)=-3.850, p=0.003$ ) were significant, but again pecking frequency was not (mean $\Delta 2.64 ; t(10)=-1.226, p=$ 0.248).
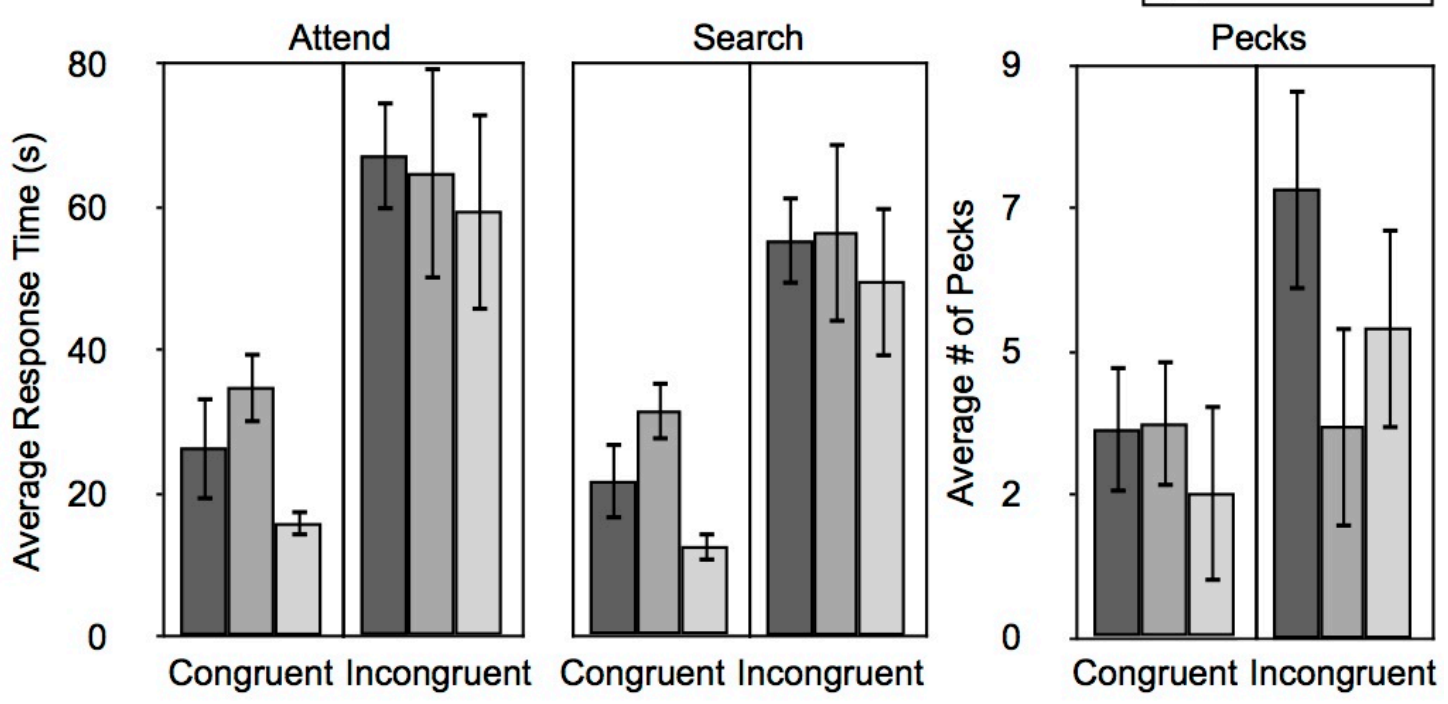

Figure 23. Experiment 1 (Prey type) - Average response in search and attendance time and number of pecks by individual insect.

Very few significant differences appeared between prey types. A One-Way ANOVA was used to compare means between conditions. The average duration of attendance (64.73s) and searching (56.55s) but not peck frequency (5.09) for 
mealworms in the incongruent condition was longer than that of locusts (AD 67.18s, SD 55.36s, PF 7.09) in the incongruent condition. Incongruent trials using earthworms had lower values in all measures (AD 59.45s, SD 49.73s, PF 4.91) than either mealworms or locusts. None of the differences in means between these three prey items were significant for either attendance duration $(F(2,30)=0.098, p=$. $907)$, search duration $(F(2,30)=0.127, p=.881)$, or pecking frequency $(F(2,30)=$ $0.536, p=.591$ ) in incongruent trials however. For congruent trials, values for trials using mealworms were greatest (AD 34.91s, SD 31.45s, 3.36), above those for trials using locusts ( $A D 26.45 \mathrm{~s}, \mathrm{SD} 21.64 \mathrm{~s}, 3.27$ ), and trials using earthworms had the lowest values in all measures (AD 15.91s, SD 12.55s, 2.27). Interestingly, while the pecking frequency was not significantly different across insects in congruent trials $(F$ $(2,30)=0.259, p=.774)$, differences in search $(F(2,30)=5.207, p=.011)$ and attendance duration $(F(2,30)=3.246, p=.053)$ across insects were both significant.

Experiment 2: Prey animacy. This experiment involved hiding events using only one type of prey (mealworms), displayed in various states (alive, dead, halved), or a small stick of an analogous size. An experimenter displayed an event where the robin found prey that was either in a consistent state or in an inconsistent state with the prey seen being dropped into the box. Significant differences resulted between the responses in congruent and incongruent trials in multiple dimensions (see Figure 24). An Independent-Samples T-Test was used to compare means between conditions. Overall, attendance duration (mean difference 14.97s; $t(86)=-1.976, p=$ 0.051 ), search duration (mean difference 12.15s; $t(86)=-2.023, p=0.046$ ) differed significantly between congruent and incongruent conditions, but peck frequency (mean difference 1.26; $t(86)=-1.353, p=0.180$ ) did not.

Prey was found either in the same state it was hidden (live-live, dead-dead); found alive after being dropped in inanimate (dead-live, stick-live), found inanimate after being dropped in alive (live-dead, live-stick), or found halved (and dead) after being dropped in whole (live-half, dead-half). Paired-Samples T-Test was used to compare means between conditions. Robins spent significantly more time in response to trials where prey was unexpectedly found inanimate ( $A D$ mean $\Delta$ $15.18 \mathrm{~s} ; t(21)=-2.213, p=0.038 ; \mathrm{SD}$ mean $\Delta 13.55 \mathrm{~s} ; t(21)=-2.390, p=0.026 ; \mathrm{PF}$ mean $\Delta 1.23 ; t(21)=-2.734, p=0.012)$ or halved $(A D$ mean $\Delta 18.77 \mathrm{~s} ; t(21)=-2.459$, 
$p=0.023 ; \mathrm{SD}$ mean $\Delta 13.55 \mathrm{~s} ; t(21)=-2.397, p=0.026$; PF mean $\Delta 2.14 ; t(21)=$ $-2.255, p=0.035$ ) with comparison to being found in the same state. While robins did spend more time on average attending (4s), searching (8s), and a higher average number of pecks $(0.50)$ in trials were prey was unexpectedly found alive with comparison to being found in the same state, none of these differences were significant $(\mathrm{AD} t(21)=-1.233, p>0.05 ;$ SD $t(21)=-1.503, p>0.05$; PF $t(21)=0.087$, $p>0.05)$.

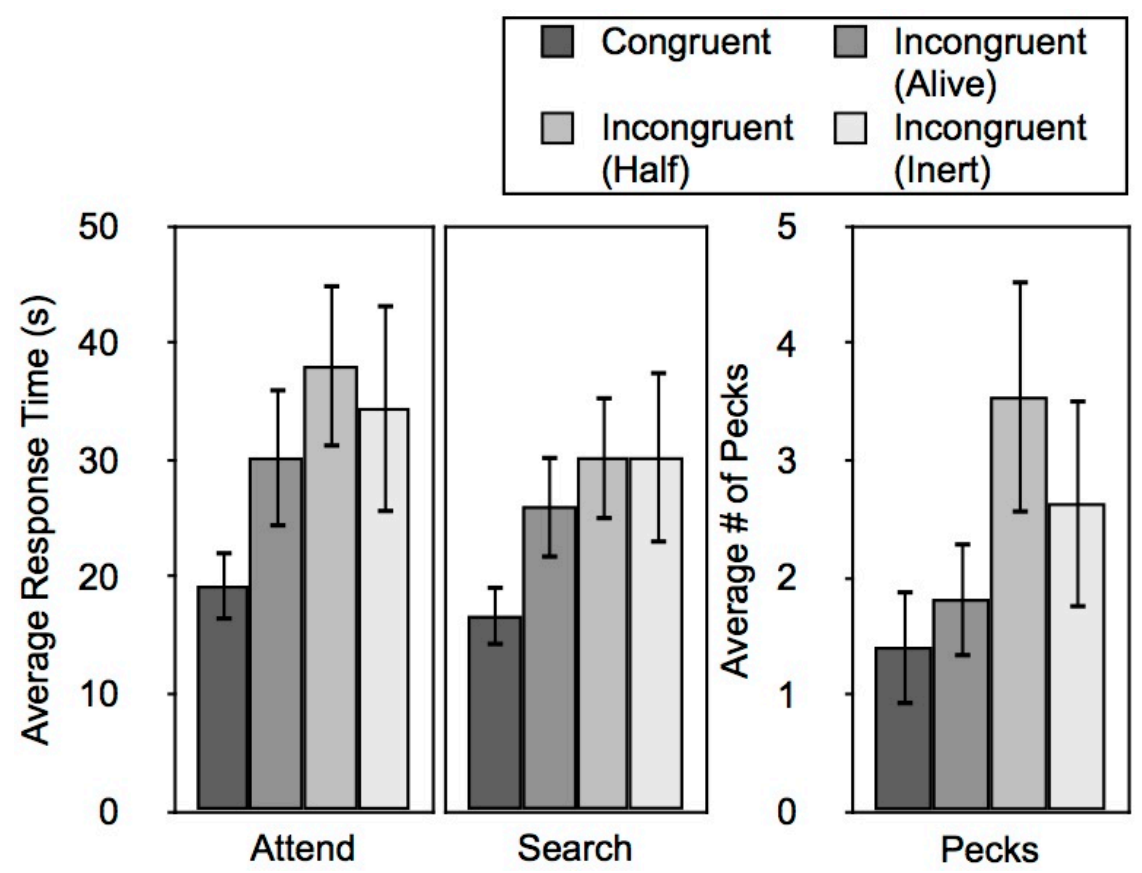

Figure 24. Experiment 2 (Prey animacy) - Average response in search and attendance time and number of pecks.

Prey destination. Taken together, the prey destination - whether it was eaten, taken away for caching or provisioning, or left in the experimental box played a significant role in the response duration (see Figure 25), and much longer response durations when the worm was eaten immediately. A One-Way ANOVA was used to compare means between conditions: prey being eaten $(\mathrm{N}=87)$, taken away $(\mathrm{N}=17)$, and left in the box $(\mathrm{N}=6)$. Trials where there was no prey found were not included. On average, all three response dimensions were all higher when prey was consumed $(A D=33.56 \mathrm{~s}, S D=28.67 \mathrm{~s}, \mathrm{PF}=2.89)$, than when prey was taken away $(A D=7.94 \mathrm{~s}, S D=7.41 \mathrm{~s}, P F=1.41)$ or left $(A D=12.67 \mathrm{~s}, S D=9.67 \mathrm{~s}, P F=0.33)$. 
When examining prey destination across all congruent and incongruent trials in both experiments, these differences appeared significant in attendance duration ( $F(2$, $107)=7.739, p=.001)$ and search duration $(F(2,107)=8.238, p=.000)$, but not pecking frequency $(F(2,107)=2.030, p=.136)$.

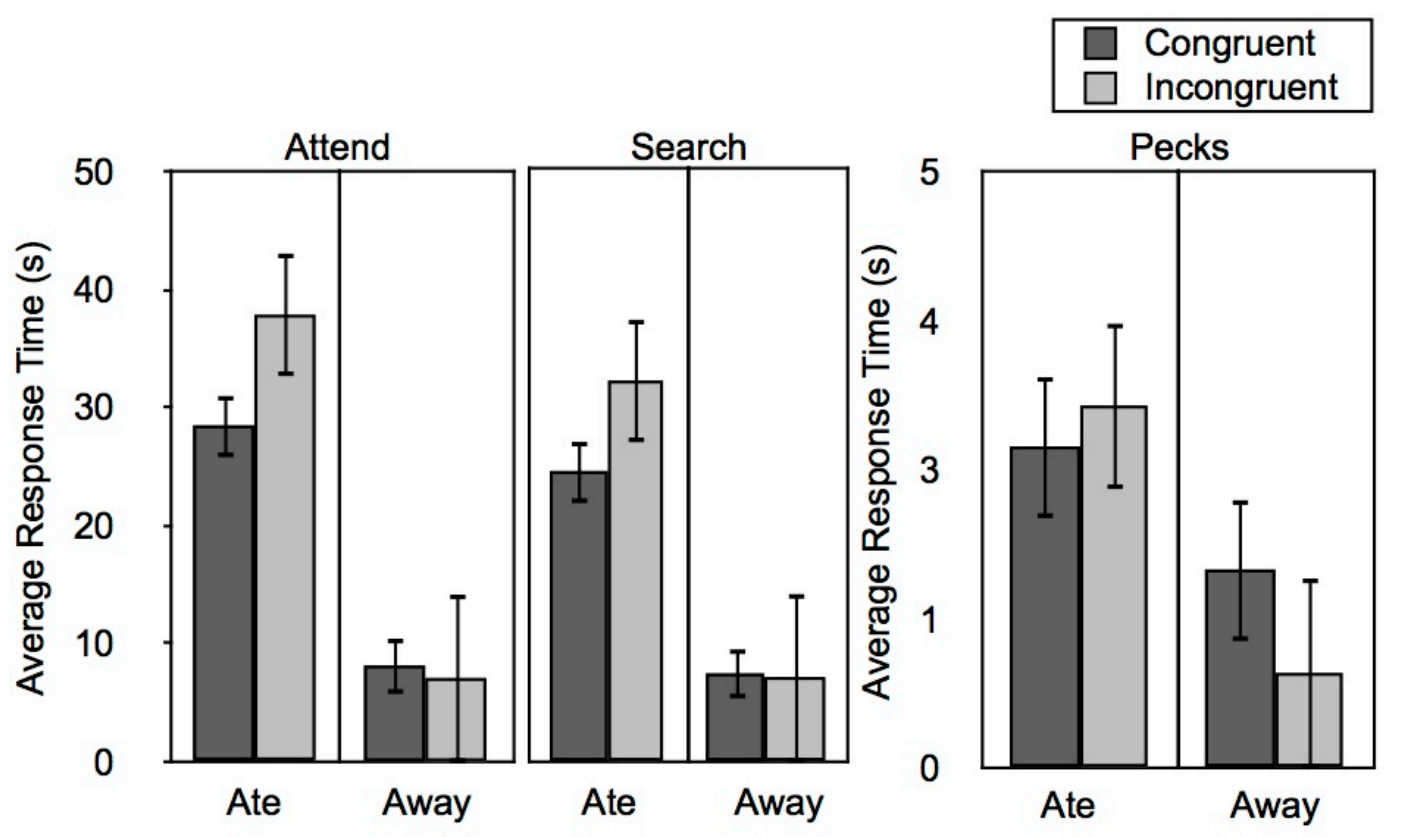

Figure 25. Overall response by prey destination - Average response in search and attendance time and number of pecks.

\section{Discussion}

The data from this study reveals salient food preference information for the North Island robin, in respect to both prey type and animacy. In Experiment 1, robins show a significantly longer search time when no insect is found (incongruent) after seeing one dropped into the box, but do not show significant differences in search time between insect types - for example, search times are not significantly longer on average when a locust goes missing than when an earthworm goes missing. While mobility of these insects varies widely, for example with locusts able to cover the greatest distance at a higher speed, but the earthworms and mealworms able to crawl through smaller spaces (and therefore gaps in an apparatus), these or other physiological details appear not to have influenced decision-making enough for a clear preference to emerge in robins' comparative search times. The type of insect does not appear to strongly influence the amount of time spent searching for a 
missing insect, in lieu of altering any other feature variables (size, colour, background).

In Experiment 2, robins show significantly longer search times for incongruent trials, where prey is revealed in a different state than when hidden (for example hidden dead and revealed alive, or hidden alive and revealed a stick), than for congruent trials, where prey is revealed in the same state as when hidden. The exception to this pattern occurs in the cases in which prey is hidden inanimate or dead and found alive. Looking at both experiments taken together, prey destination played a significant role in the search times of the robins, as they spent significantly longer searching when prey was immediately consumed than when taking it away to cache or provision.

Given the nature of caching highly perishable food such as insects, a keen response to the state of animacy a prey is hidden in, as well as when that state changed (prey stunned or killed) could be a vital aspect of hunting and caching strategy for a species like North Island robins. Alexander et al. (2005) note that when robins are caching, prey is frequently injured but not killed. Whether this is accidental, or intentional, is unclear, but as a caching strategy, it is beneficial for storing highly perishable food, as already dead prey will spoil faster. A battery of experiments show that Scrub Jays are sensitive to exactly these types of features the perishability and point in time a food item was stored (Clayton \& Dickinson, 1998, 1999), and additionally whether and which conspecifics were watching (Dally et al., 2005; Emery et al., 2004), as well as the length of time lapsed between storing and re-accessing a given cache.

Robins' natural diet includes a wide variety of invertebrates (Menzies \& Burns, 2008), including slugs, stick insects (Phasmatodea) (Jewell \& Brock, 2002), earthworms (Lumbricidae) (Lee \& Zelanda, 1959), and weta (Orthoptera: Anostostomatidae) (Gibbs, 1998), among others; even prey larger or heavier than itself (Daugherty, Gibbs, \& Hitchmough, 1993; Powlesland, 1981). The mealworms presented here in Experiment 2, and certain conditions in Experiment 1, are not part of the North Island robin's natural diet, and the insects presented comprise a limited array items. A broader assessment of preference for a naturally encountered prey, in relation to size (dimensions and volume), features (exoskeleton, limbs, defences), colour and other variables would inform a more complete picture in order to better 
understand robin's behaviour with respect to insect animacy as a whole, and it's variation across differing insect species. With such a battery, it is possible that stronger preferences or expectations in VoE events might arise between varying types of insects based on physical features or mobility.

Whether robins' pattern of longer search response for unexpected dead, partial or inanimate items is indicative of a broader suite of behaviours that applies to more abstract tasks (points of light or 2D animations) is an open question. While motion appears to be a natural category for trained pigeons in that they can discriminate between video footage of moving and non-moving pigeons using only motion as a cue (Dittrich \& Lea, 1993), only some pigeons appear able to transfer the ability to discriminate biological motion (walking, pecking) onto displays of points of light as well (Dittrich, Lea, Barrett, \& Gurr, 1998). Robins' slightly longer reaction time in cases where the item revealed was unexpectedly alive after being hidden dead or inanimate compared to trials where the state remained the same could in part be due to these adult robins' prior exposure to experimental trials where multiple quantities of mealworms were presented in a choice task (see Chapter 2), or more than one worm was placed in the VoE box in a computation task (see Chapter 4).

The present study provides a precursor upon which to develop a more detailed understanding of the cognition behind New Zealand robin's caching strategies, prey selection, memory, and the role of environmental biodiversity and surrounding movement. The North Island robins' sensitivity to differing states of animacy in prey sheds light on salient visual information that may be attended to even in tasks aimed at other cognitive domains, such as numerosity or spatial memory. Additional followup tasks such as presentation of virtual insect animations might further be revealing of how generalizable such behaviours are, or whether recognition of self-propulsion vs. causal motion plays a role in caching and cache protection strategy as it relates to the animacy and therefore decay of such perishable prey. 
CHAPTER 8: General Discussion 
The six experiments detailed here provide evidence that North Island robins (Petroica longipes) have both a sophisticated number sense, and a basic awareness of heterospecifics' eye and limb visibility as salient to social cues to pilfering. It is hoped that these studies have accomplished at least three purposes. First, these studies lay the groundwork for further cognition research particularly with this unique species, but also with other native New Zealand species. Second, they add to the body of knowledge encompassing the ecology, behaviour and cognition in an endemic species of bird to New Zealand. Third, they contribute to the collection of research that shapes the understanding of mechanisms that underly basic developmental cognition across species.

\section{Summary}

The first three studies presented here focused on the North Island robins' response to manipulation of numerical quantity. Study 1 explored the Relative Quantity Judgements (RQJs) between both small numbers of prey (totalling 5-30) and large numbers of prey (totalling 36-120). Experiment 1 presented trials in which worms were sequentially dropped and hidden, whereas Experiments 2 and 3 presented trials where worms were simultaneously dropped and remained visible. The data found that robins were successfully able to discriminate between unusually large quantities without a significant effect of ratio. In trials with numbers considerably beyond the signature limit $(<4)$ described as characterising a number representation system, such as those in Experiment 3 (36-120 total worms per trial), a ratio effect was observed. Study 2 focused on a quantity discrimination task, in which summation of items was spatially distributed across two separate arrays, containing 7 wells each. Between 1 and 5 worms were hidden in each array before allowing robins to choose retrieval of all worms in one of the two arrays. Results found that while robins performed above chance with the small numbers presented, percentage success was lower overall than for comparable comparisons in Study 1 , suggesting higher cognitive demand for quantities that are spatially distributed in comparison with a single, continuous location. Study 3 presented the addition and subtraction of small numbers of prey items in a Violation of Expectancy (VoE) design, altering the number of prey items in Experiment 1, and the type of prey (mealworm or waxworm) in Experiment 2. Robins' reactions to computation were 
measured looking at the time spent generally attending to the apparatus, actively searching the VoE box, and pecking at the box or cover. The data found that robins displayed these attention and search behaviours longer when presented with a mathematically incongruent scenario.

The final three studies presented here all explored robins' response to various features of agency. Study 4 examined robins' response to the visibility of human eyes when faced with the opportunity to pilfer prey from one of two experimenters. Results revealed that robins were sensitive to the presence of human gaze in all conditions but one, and preferred to pilfer the mealworm from the experimenter whose eyes were obscured. In Study 5, the response to human limb visibility was measured in a similar choice task, where robins were presented with the opportunity to 'steal' prey from one of two human experimenters. In Experiment 1, one or more 'limb regions' (arms, legs, nose/mouth) were obscured using an opaque cloth, in which the region covered for one experimenter was left exposed in the other. Experiment 2, in contrast, used a wooden plank to obscure the robins' view, and presented trails where only a single limb region of one experimenter was exposed, and none were exposed for the other. The data shows that robin choice was significantly influenced by the visibility of the experimenter's limbs, but did not appear to avoid one limb region more than the others. Study 6 used two different VoE experiments to investigate the response of robins' to the manipulation of presence and absence comparing prey type, or replacement of items in differing states of animacy. Responses were observed using the same measures analysed in Study 2 (general attendance time, active search time, and number of pecks at the apparatus). Experiment 1 presented three different prey types; locusts, mealworms and earthworms, and allowed robins to either find the same insect that was hidden, or find no insect. Subjects responded by searching longer when the insect was found than when it was missing, but results saw no significant difference in length of response when comparing different insect types (for example, robins did not search significantly longer for a missing locust than a missing mealworm). Experiment 2 presented only mealworms (or small sticks in some conditions), but altered the state in which they were found (alive, dead, halve, inanimate stick) with comparison to that in which they were hidden. Results indicated that in each case, robins searched longer when the state of animacy was changed than in conditions where it remained 
the same, suggesting that robins display an awareness of the state of animacy in cached prey.

This body of research does not delve into the heart of nativist-empiricist or domain generalist-specifist debates; those are theoretical problems of a scale not intended to be resolved here. It should however, provide behavioural information within a relevant ecological context, that may be salient to forming a more complete picture of a suite of behaviours that could help to describe the possible cognitive mechanisms driving shared, repeatable response present in this population of birds, and possibly in many other species as well. The evidence provided by the suite of experiments described here provides observable patterns in data that North Island robins behave by responding to external stimuli in ways which appear in line with the behaviours seen in many other animals and human infants which characterise Spelke's Core Knowledge (CK) hypothesis (Spelke, Breinlinger, Macomber, \& Jacobson, 1992; Spelke, 2000). It does, however, differ in the parameters of such responses in some cases - for example, in Study 1, where ratio is not predictive of discrimination of quantities immediately beyond 4 (appearing instead with much larger values), or in Study 4, where robins fail to differentially pilfer worms from an experimenter facing $90^{\circ}$ away from the worm as opposed to one fully facing the worm.

In developmental and comparative cognition, behaviour is largely used as a window into what underlying processes in the mind might be driving a pattern of responses that can be seen developing in a predictable way across a lifespan, or in multiple species across evolution. While the success of using behaviour as a vehicle to describe the cognitive architecture of any given participant, let alone an entire species or groups of them, is challenging (Penn, Holyoak, \& Povinelli, 2008), the importance of experimentally examining such behaviour within varying contexts is clear. There are certainly theories of cognitive development that present strong alternatives to the Core Knowledge theory developed by Elizabeth Spelke and colleagues $(2000 ; 2007)$, and provide somewhat differing frameworks with which to investigate both development of behaviour and cognition in human infants as well as other species. Core knowledge theory (see Chapter 1), in the context that it is taken here, is used as a foundation on which to build applicable field experiments to explore North Island robin behaviour in its natural ecological context. Although the 
theory itself posits that such cognitive domains are inherently present at birth (Spelke, 2000; Spelke \& Kinzler, 2007), the aim of this particular set of studies was not necessarily to provide either support or refute this claim, and to attempt to do so using a wild population of North Island robins would be an extremely difficult feat, given their ecology. Robins raise 2-3 clutches of young in a season, and offspring start hunting, caching and searching for their own mate and territory well within the first year of life; transitioning from parental provisioning of food to independent competitors within an extremely short time-frame. Acquiring behavioural responses to given scenarios, particularly choice tasks, would be next to impossible to successfully determine in nestlings or young fledglings, who would not necessarily engage in recovering insect prey themselves, especially with a parent present, let along accurately distinguishing what factors may have differentiated such responses in nestlings. While age is indeed a very interesting factor in what can be a relatively long-lived passerine in protected areas (a few of the subjects of this study were well above 5 in age) (Taylor, Boessenkool, \& Jamieson, 2008), it is not something focused on specifically in these studies.

Another point is that using the CK framework as has been done here does not necessarily preclude robins from acting or reacting on the basis of either perceived stimulus or unseen processes that are unforeseen by experimenters here or in previous works (Hunt et al., 2008; Menzies \& Burns, 2008). Robin behaviour in this collection of studies is indicative of a basic, and in some cases more complex, ability to act systematically within the context of certain information: they could be called specialists in discerning quantities when it comes to prey inventory, and are sensitive to visual cues such as eyes and limbs, which sometimes carry socially significant information, even in heterospecifics that are evolutionarily distant and invasive in the native environment. These studies do not, however, broach more complex cognitive abilities that frequently come under debate (Penn \& Povinelli, 2007; Penn et al., 2008; Povinelli \& Vonk, 2004), such as transitive interference or theory of mind (Bugnyar, 2007; Pika \& Bugnyar, 2011). 


\section{Interpretation}

In many ways, the studies presented here can be seen as an exercise in behavioural ecology, viewed through the window of developmental cognition. While information derived by comparative studies such as these, and their application as a window into the evolution of behaviour and any accompanying cognitive mechanisms in the brain, is by its very nature quite speculative. Just as this area can be better informed by comparing suites of behavioural and genetic information for two very similar but slightly different sub-species, and looking contextual and functional differences; so can it be informed by doing the same for very evolutionarily disparate species, like those isolated by New Zealand evolutionary history.

There are certainly many ways in which the theories discussed above are not necessarily mutually exclusive; the presence of innate mechanisms aiding in the categorical absorption and analysis of information in given domains does not preclude memory and external stimuli acting to process information and using rules once experience allows, or eradicate the influence that social transfer of information might play on the development and use of more complex cognitive mechanisms. The aim in this instance is not to prove or disprove exclusivity or application of any or all such theories either to this particular species or life as a whole, but to borrow one as a framework within which to begin building behavioural evidence that may serve to illuminate basic cognitive function in an evolutionarily unique species. Because of the nature of this particular species - the North Island robin - the Core Knowledge framework provided the most ecologically salient basis upon which to develop a set of feasible field experiments; even in cases where the evidence elicited did not necessarily support the defining characteristics of this theoretical framework as it exists today.

Number. Cohesion has proven to be an essential feature in discrimination of quantities (Cheries, Mitroff, Wynn, \& Scholl, 2008; vanMarle \& Wynn, 2011; vanMarle \& Wynn, 2006), and may have played some role in influencing differing results between the hidden sequential presentation of worms in Experiment 1 and the visible poured presentation in Experiment 2 of the present study. Infants require a larger ratio difference to discriminate quantities of a non-solid substance, such as Cheerios $^{\mathrm{TM}}$ (at least 1:4) compared to quantities of discrete objects, such as crackers (1:2). Strikingly, when Cheerios ${ }^{\mathrm{TM}}$ were then presented as discrete, individually 
placed objects rather than poured (so they no longer appeared as a non-countable 'substance') infants were successful at the 1:2 ratio they previously had failed (2011). For robins, this may well account for a slightly lower accuracy in Study 1, in the condition in which worms were poured in the present experiment; where robins fall to chance in the larger 0.50 ratio (8 vs. 16) in Experiment 2 (50\%), but not in the same comparison when it is sequentially placed in Experiment 1 (67\%) (see Table 2). Failure of robins to rely on ratio to distinguish numbers in the face of the more cognitively challenging task in Study 2 , where comparisons were spaced out across two arrays, is highly interesting in light of the lower success rates in choosing the larger quantity. The distinct difference in percentages of success between trials where the smaller quantity was 1 or 2 may be indicative that where quantity information is confounded by space, time or other variables, robins use a more basic single-plural distinction, like that seen in some quantity research with rhesus monkeys (Barner et. al., 2008). A mechanism allowing strong singular-plural differentiation would allow robins to more successfully make quick caching and recovery decisions necessary, even in the face of complex environmental sensory and information that surround such decision making, for example, the animacy state the worm was cached in (dead, paralysed, alive), the time and place that it was cached, weather conditions, prey type (earthworm, weta), and likelihood of pilfering. The success of robins in recognising basic addition and subtraction of items in Study 3 raises the question of how great or small a role capacities like attentiveness to individual physical features of each prey item, and whether that might allow them to track individual prey or pieces of prey (when large prey is divided). Study 2, where between 3 and 6 worms were placed amongst 14 separate locations, and Study 3 , where search time was longer when a number or type of worm went missing, both lead to the question of whether information such as the order in which prey is cached (and therefore the age or 'freshness') influences any number of natural behaviours: Does caching strategy in the presence of conspecifics change? Robins will often aggress a mate if they are found pilfering a cache (Menzies, 2008). Is a pilfered item less likely to result in aggression if it was the least recent item cached? Is the choice of item pilfered thusly influenced? Only a detailed continuation of both ecological and experimental studies of such scenarios will reveal the subtleties of this decisionmaking process, and whether it is influenced by an underlying numerical system. 
The analysis of the Study 1 revealed that robins appear to use two systems for coping with numbers. An object indexing system and a ratio-based analog magnitude system each produce an apparent and distinct 'signal' across the number scale. The odds ratio for the object-file system hovered just below 1 across all three experiments $(\operatorname{Exp}(B) s$ ranged from 0.986 to 0.926$)$ creating a signature of a gradual decline as objective number (total set size) increased, a signal that was insignificant for sets below 16. The signature which emerged for a ratio-based system was expressed by a very low odds ratio across all experiments $(\operatorname{Exp}(B) s$ ranged from 0.287 to 0.110 ), showing a drastic decline in probability of success as ratio increased.

The strength of the object indexing system is in providing a more accurate mechanism based upon enumerating distinct objects; it attempts to process objects as discrete items regardless of magnitude. The recent study with infants by vanMarle and Wynn (2011) speculates on exactly this interplay between mechanisms in infants, reminding us that the analogue magnitude system was initially based on data accounting for rats and pigeons perception of continuous stimuli such as time (duration) and number (Meck \& Church, 1983) and that analogue magnitude representations themselves are also continuous, not discrete. They also suggest the possibility that estimation of substances is more variable than enumerating objects as a non-solid stimulus can change shape, dimension or break apart upon movement - and that two mechanisms are likely to underlie the infant's quantification abilities. It is compelling that for both robins and human infants, the same noncohesive material in each case (mealworms and Cheerios ${ }^{\mathrm{TM}}$ respectively) can be perceived and possibly represented as either a continuous substance or as discrete items depending on context and presentation; and that in the case of robins especially, working memory and ecology appears to extend the item indexing limit typically associated with mammals tracking discrete items. Using New Zealand Robins as a model system invites us to understand the representations underpinning numerical cognition in a new light.

Agency. Speaking specifically to research on chimpanzee behaviour, Povinelli et al. (2004) raises the issue that any conclusions made about their ability to reason about unobservable mental states is still a matter of controversy. It is understood by many researchers, primate and otherwise, that not only is there a system that deals 
with social cognition in such a way that allows for representation and reasoning about behaviour, but that this system also then places these behavioural representations within a framework of varying mental states (Bekoff, Townsend, \& Jamieson, 1994; Clayton, Yu, \& Dickinson, 2003; Bekoff, Allen, \& Burghardt, Eds., 2002; Pepperberg, Hurley, \& Chater, 2005; Tomasello, Call, \& Hare, 2003). Povinelli also argues that the present experimental paradigm makes it impossible to resolve this confounding issue in comparative cognition research.

Regardless of how such studies might be interpreted, it is hoped that the present research might be used as a foundation upon which to carry out further, more complex studies in the future. The present studies do not attempt to delve into something approaching the complexity of Theory of Mind (ToM), but rather, focus on what could be considered more elementary building blocks of behaviour. There is no conclusive evidence in Study 4 (Chapter 5) that robins' response to human gaze is indicative of an intricate understanding of human intention (if that were the case, it might be more apparent to them that the human experimenters have little desire to consume or protect the mealworms presented). While we can speculate as to whether some kind of 'like me' mechanism is functioning, only further study could give real evidence of such a system actively in use. At present, data regarding response to eye gaze or limb visibility (Study 5) is more indicative of a basic awareness of the function of human physiology, whether acquired through interaction with humans, or some combination of other means. This is an intriguing feat in and of itself, regardless of speculation on underlying mental representations, given that rapid response to eye-like shapes in other species is typically associated with antipredatory response (Burger, Gochfeld, \& Murray, 1992; Hampton, 1994), given that North Island robin populations that are isolated from introduced predators display no anti-predatory response in their presence (Whitwell et al., 2011).

\section{Cognition in the Field}

Suggesting that ecological validity matters for behaviour and cognition research is not to say that lab based experimentation involving training or other artificial environments or stimuli is not useful, but that animal behaviour studies set in their natural context are just as relevant for cognition research as it might be for human social, cultural or anthropological research (Emery \& Clayton, 2004, 2009; Emery, 
Clayton, Bolhuis, \& Giraldeau, 2005). Experimental research on rats or pigeons has served to inform many of the concepts underpinning the way cognition is understood (Cook, 2000; Koehler, 1941; Meck \& Church, 1983; Meck, Church, \& Gibbon, 1985). Bringing ecological context to this information is the other side of the same coin; the ability to look at the functionality of these behavioural traits, and its variation in different contexts better informs the conception of mechanisms that may be underlying them. Zebra finches (Taeniopygia guttata), for example, are widely used for both behavioural and neurocognitive research, but captive and wild populations may show very different responses to similar measures and even differing genetic variation (Forstmeier, Segelbacher, Mueller, \& Kempenaers, 2007; Gilby, Mainwaring, Rollins, \& Griffith, 2011).

The present study places these experimental paradigms in the natural ecological context of the species in focus, the North Island robin. In working with a wild population of endemic birds in native forest, on their own territories, some measure of experimental control is necessarily ceded in the design and execution of the experiment. By the same token however, placing such experiments in context with the subjects' natural environment allowed certain confounds to be minimised as well. Training, in the true sense of the word, was not necessary for robins to learn to access the artificial caches used in the experimental designs here. Brief familiarisation with the apparatus, leather cover, and bait were enough to allow birds to recognise mealworms (which are not naturally found in native New Zealand forest) as desired prey items, and remove the leather flap in the same manner that they might overturn leaf litter on the forest floor. In this sense, preparatory methodology is not unlike studies with precocial, imprinting birds where brief initial familiarisation suffices rather than extensive repetitive training at a task (Rosa-Salva, Regolin, \& Vallortigara, 2010; Salva, Farroni, Regolin, Vallortigara, \& Johnson, 2011; Vallortigara \& Regolin, 2006; Vallortigara et al., 2005).

Although that may be the case, research to this point has revealed an interesting suite of behaviours that beg continued investigation. This is particularly true given the unique position this threatened species finds itself in, in several respects: First, the evolutionary isolation of the species endemic to New Zealand as a result of it's early geographic divide from the other continents gives rise to behaviours and genetic diversity unlike those on other continents (Daugherty et al., 1993). Second, 
New Zealand robin subspecies are the only endemic species that hoard food in their wild state. Lastly, the approachability and accessibility of this species is nearly unparalleled, outside of other isolated island species that also lack anti-predatory responses to humans. Further research necessary to realise this potential - it is hoped that this series of experiments will serve as a launching point for a more indepth look at behaviour, ecology and cognition from multiple perspectives. A number of questions arise, that could apply to the studies presented here as a whole. How do these behaviours vary under differing levels of human or introduced predator exposure? What level of plasticity exists in a given behavioural response depending on variations biodiversity exposure and geographical isolation? What role might evolution and co-evolution play, and how might comparison with invasive species assist in answering that? What behavioural range can be seen both within and across different ecological settings, and what does that say about the sensory input that a given cognitive mechanism flexibly deals with? How could such mechanisms adjust to these differences, and what commonalities do not alter with ecological context? Will these behaviours transfer to virtual displays of information, such as animations on a screen surface? Does familiarisation with certain environmentally dictated natural limitations, such as typical cache inventory (Alexander et al., 2005) or territory dimensions influence the cognitive abilities or limitations in those domains (e.g. numerosity or geospatial information)?

As a whole, the results of these six studies show that while supporting core features defining CK in many ways, unique results in some aspects of the abilities of this biologically naïve and evolutionarily isolated wild species sheds new light on our growing understanding of the shared basis of cognition. It is certainly possible that further in-depth study arising as a continuation of the present behavioural research in North Island robins will continue to reveal both complementary and conflicting evidence when viewed in light of the current understanding of CK. In doing so, commonalities and differences arising in avian performance of core developmental tasks, with the abilities of other nonhuman species can offer new insights into sweeping evolutionary theories that underpin basic cognitive mechanisms. 


\section{Appendix A: Participant Information}

Information about experimental participation, approximate age, and sex of North Island robins observed interacting in these studies. Approximate current age is provided; age at testing varied based on date of data collection.

\begin{tabular}{|c|c|c|c|c|c|c|c|c|c|}
\hline $\begin{array}{l}\text { Bird ID } \\
\text { Band }\end{array}$ & Sex & $\begin{array}{l}\text { Banding } \\
\text { Date }\end{array}$ & $\begin{array}{c}\text { Approx. } \\
\text { Age }\end{array}$ & $\begin{array}{c}\text { Study } \\
1\end{array}$ & $\begin{array}{c}\text { Study } \\
2\end{array}$ & $\begin{array}{c}\text { Study } \\
3\end{array}$ & $\begin{array}{c}\text { Study } \\
\quad 4\end{array}$ & $\begin{array}{l}\text { Study } \\
5\end{array}$ & $\begin{array}{c}\text { Study } \\
6\end{array}$ \\
\hline BK-OM & Male & 2008 & $>4$ & $\checkmark$ & & $\checkmark$ & $\checkmark$ & $\checkmark$ & $\checkmark$ \\
\hline BM-BB & Male & 2009 & 3 & $\checkmark$ & & & $\checkmark$ & & \\
\hline BM-BfP & Juvenile & 2009 & 3 & $\checkmark$ & & & & & \\
\hline BM-BW & Juvenile & 2009 & 3 & $\checkmark$ & & & & & \\
\hline BM-YW & Male & 2009 & 3 & $\checkmark$ & & $\checkmark$ & $\checkmark$ & $\checkmark$ & $\checkmark$ \\
\hline dBW-OM & Male & 2008 & $>4$ & $\checkmark$ & & & & & \\
\hline fPB-OM & Female & 2008 & $>4$ & $\checkmark$ & & & & & \\
\hline fPM-WR & Female & 2009 & 3 & $\checkmark$ & & & $\checkmark$ & $\checkmark$ & \\
\hline fPM-YpB & Male & 2009 & $>3$ & & & & $\checkmark$ & $\checkmark$ & \\
\hline fPM-YR & Female & 2009 & $>3$ & $\checkmark$ & & $\checkmark$ & $\checkmark$ & $\checkmark$ & $\checkmark$ \\
\hline GR-BM & Juvenile & 2009 & 3 & $\checkmark$ & & & & & \\
\hline GR-OM & Male & 2008 & $>4$ & $\checkmark$ & & $\checkmark$ & $\checkmark$ & $\checkmark$ & $\checkmark$ \\
\hline GW-BM & Juvenile & 2009 & 3 & $\checkmark$ & & & & & \\
\hline GWY-BM & Juvenile & 2009 & 3 & $\checkmark$ & & & & & \\
\hline KY-OM & Male & 2008 & $>4$ & & & & $\checkmark$ & $\checkmark$ & \\
\hline OK-OM & Male & 2008 & $>4$ & $\checkmark$ & & & & & \\
\hline OM-BG & Male & 2008 & $>4$ & $\checkmark$ & & $\checkmark$ & $\checkmark$ & & \\
\hline OM-fPB & Male & 2008 & $>4$ & & & & $\checkmark$ & $\checkmark$ & \\
\hline OM-GO & Female & 2008 & $>4$ & $\checkmark$ & & & & & \\
\hline OM-RK & Male & 2008 & $>4$ & $\checkmark$ & $\checkmark$ & $\checkmark$ & $\checkmark$ & $\checkmark$ & $\checkmark$ \\
\hline OM-RO & Female & 2007 & $>5$ & $\checkmark$ & & $\checkmark$ & $\checkmark$ & $\checkmark$ & \\
\hline OM-RR & Female & 2008 & $>4$ & $\checkmark$ & & & $\checkmark$ & $\checkmark$ & \\
\hline OM-WK & Female & 2008 & $>4$ & $\checkmark$ & & $\checkmark$ & $\checkmark$ & $\checkmark$ & $\checkmark$ \\
\hline OM-YK & Male & 2008 & $>4$ & $\checkmark$ & $\checkmark$ & $\checkmark$ & $\checkmark$ & $\checkmark$ & $\checkmark$ \\
\hline OM-YP & Female & 2008 & $>4$ & $\checkmark$ & & & & & \\
\hline OM-YW & Male & 2008 & $>4$ & $\checkmark$ & & $\checkmark$ & $\checkmark$ & $\checkmark$ & $\checkmark$ \\
\hline OW-OM & Unknown & 2008 & $>4$ & & & & & $\checkmark$ & \\
\hline RdB-fPM & Female & 2009 & $>3$ & $\checkmark$ & & & $\checkmark$ & $\checkmark$ & \\
\hline RfP-fPM & Male & 2009 & $>3$ & $\checkmark$ & & $\checkmark$ & $\checkmark$ & $\checkmark$ & \\
\hline RG-fPM & Male & 2009 & $>3$ & $\checkmark$ & & $\checkmark$ & & & \\
\hline RM-OB & Male & 2007 & 5 & $\checkmark$ & $\checkmark$ & $\checkmark$ & $\checkmark$ & $\checkmark$ & $\checkmark$ \\
\hline RR-BM & Juvenile & 2009 & 3 & $\checkmark$ & & & & & \\
\hline RR-fPM & Male & 2009 & $>3$ & $\checkmark$ & & $\checkmark$ & $\checkmark$ & $\checkmark$ & \\
\hline RW-BM & Male & 2009 & 3 & & & & & $\checkmark$ & \\
\hline RW-OM & Male & 2007 & 5 & & $\checkmark$ & $\checkmark$ & $\checkmark$ & $\checkmark$ & $\checkmark$ \\
\hline RY-fPM & Male & 2009 & $>3$ & $\checkmark$ & $\checkmark$ & $\checkmark$ & $\checkmark$ & $\checkmark$ & $\checkmark$ \\
\hline WfP-BM & Juvenile & 2009 & 3 & $\checkmark$ & & & & & \\
\hline WM-WB & Male & 2003 & 9 & $\checkmark$ & & $\checkmark$ & & & \\
\hline WO-GM & Male & 2004 & 8 & $\checkmark$ & & & & & \\
\hline YG-OM & Male & 2007 & $>5$ & $\checkmark$ & & & $\checkmark$ & $\checkmark$ & \\
\hline YR-KM & Male & 2001 & 11 & $\checkmark$ & & & $\checkmark$ & $\checkmark$ & \\
\hline YW-OM & Female & 2008 & $>4$ & $\checkmark$ & & & & $\checkmark$ & \\
\hline
\end{tabular}




\section{Appendix B: Zealandia - Karori Sanctuary}

The visitor's map of Zealandia: Karori Sanctuary

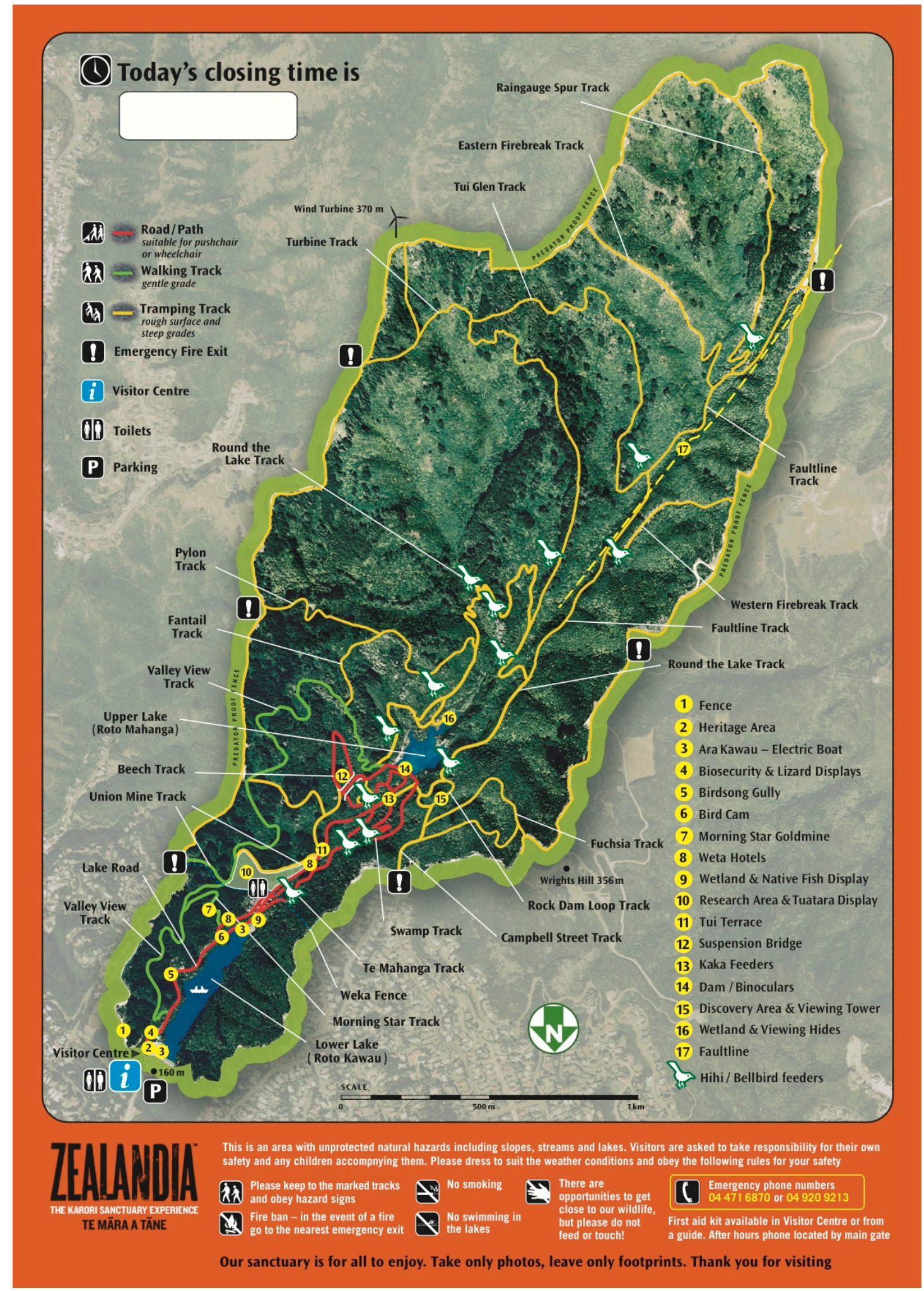

(Credit: Zealandia - The Karori Sanctuary Experience) 


\section{Appendix C: Supplemental Materials Online}

\section{Sample Trial Videos ${ }^{4}$}

Study 1: Discrimination of Large and Small Quantities

Experiment 1 (Quantities up to 16, hidden) - Ex. 6 vs. 8 (choice 8 )

Experiment 2 (Quantities up to 16, visible) - Ex. 16 vs. 8 (choice 16)

Experiment 3 (Quantities up to 64, visible) - Ex. 8 vs. 64 (choice 64)

Study 2: Quantity Discrimination Across Multiple Arrays

Example - 1 vs. 3 (choice 3 )

Example - 2 vs. 5 (choice 5 )

Study 3: Addition and Subtraction

Experiment 1 (Item Number)

Demonstration - Preparing Incongruent Trial

Congruent Trial - Ex. $1+1=2$

Incongruent Trial - Ex. 3-1 $1 \neq 1$

Experiment 2 (Item Category)

Demonstration - Preparing Incongruent Trial

Congruent Trial - Ex. $1 \mathrm{~m}+1 \mathrm{x}=1 \mathrm{~m}+1 \mathrm{x}$

Incongruent Trial - Ex. $1+1 \neq 1 \mathrm{~m} 1 \mathrm{x}$

Study 4: Response to Human Gaze

Condition 1 - Body Opposite

Condition 5 - Face Covered

Study 5: Response to Human Capability

Experiment 1 (Cloth) - Condition 1

Experiment 2 (Plank) - Condition 4

Study 6: Perception of Prey Animacy

Experiment 1 (Prey Type) - Condition 6

Experiment 2 (Prey State) - Condition 7

\footnotetext{
4 For access to these videos, please click on the link and type in the password:

AlexisThesisVUW2012. When accessing the site manually, type the following URL: https://vimeo.com/ album/2077882 for a full list of the videos above, and choose the appropriate video from the selection shown.
} 


\section{Bibliography}

Abramson, J. Z., Hernández-Lloreda, V., Call, J., \& Colmenares, F. (2011). Relative quantity judgments in south american sea lions (Otaria flavescens). Animal Cognition, 14(5), 695-706. doi:10.1007/s10071-011-0404-7

Addessi, E., Crescimbene, L., \& Visalberghi, E. (2008). Food and token quantity discrimination in capuchin monkeys (Cebus apella). Animal Cognition, 11(2), 275-282. doi:10.1007/s10071-007-0111-6

Agrillo, C., Dadda, M., \& Bisazza, A. (2007). Quantity discrimination in female mosquitofish. Animal Cognition, 10(1), 63-70. doi:10.1007/s10071-006-0036-5

Agrillo, C., Dadda, M., Serena, G., \& Bisazza, A. (2008). Do fish count? Spontaneous discrimination of quantity in female mosquitofish. Animal Cognition, 11(3), 495-503. doi:10.1007/s10071-008-0140-9

Agrillo, C., Regolin, L., \& Vallortigara, G. (2004). Can young chicks take into account the observer's perspective? Perception, 33, 110-111.

Aguiar, A., \& Baillargeon, R. (1999). 2.5-Month-old infants' reasoning about when objects should and should not be occluded. Cognitive Psychology, 39(2), 116-157.

Alexander, L., Duthie, C., Fyfe, J., Haws, Z., Hunt, S., Montoya, I., \& Van Horik, J. (2005). An experimental evaluation of food hoarding by North Island robins (Petroica australia longipes). Notornis, 52(3), 138.

Allan, S. E., \& Blough, D. S. (1989). Feature-based search asymmetries in pigeons and humans. Perception \& Psychophysics, 46(5), 456-464.

Armstrong, D. P., \& Ewen, J. G. (2002). Dynamics and viability of a New Zealand robin population reintroduced to regenerating fragmented habitat. Conservation Biology, 16(4), 1074-1085.

Armstrong, D. P., Davidson, R. S., Dimond, W. J., Perrott, J. K., Castro, I., Ewen, J. G., \& Taylor, J. (2002). Population dynamics of reintroduced forest birds on New Zealand islands. Journal of Biogeography, 29(5-6), 609-621.

Armstrong, D. P., Raeburn, E. H., Lewis, R. M., \& Ravine, D. (2006a). Estimating the viability of a reintroduced New Zealand robin population as a function of predator control. Journal of Wildlife Management, 70(4), 1020-1027.

Armstrong, D. P., Raeburn, E. H., Lewis, R. M., \& Ravine, D. (2006b). Modeling vital rates of a reintroduced New Zealand robin population as a function of predator control. Journal of Wildlife Management, 70(4), 1028-1036.

Armstrong, N. (2011). Number discrimination in New Zealand robins (Petroica australis). Thesis, Wellington, New Zealand. 
Atkinson, I. A. E., \& Millener, P. R. (1991). An ornithological glimpse into New Zealand's pre-human past. Acta XX Congressus Internationalis Ornithologici, 1, 129-192.

Baker, M. C., Stone, E., Baker, A. E. M., Shelden, R. J., Skillicorn, P., \& Mantych, M. D. (1988). Evidence against observational learning in storage and recovery of seeds by black-capped chickadees. The Auk, 492-497.

Balda, R. P., \& Kamil, A. C. (1992). Long-term spatial memory in Clark's Nutcracker, Nucifraga columbiana. Animal Behaviour, 44(4), 761-769.

Barner, D., Wood, J. N., Hauser, M. D., \& Carey, S. (2008). Evidence for a nonlinguistic distinction between singular and plural sets in rhesus monkeys. Cognition, 107(2), 603-622.

Baron-Cohen, S., Wheelwright, S., \& Jolliffe, T. (1997). Is there a "language of the eyes"? Evidence from normal adults, and adults with autism or asperger syndrome. Visual Cognition, 4(3), 311-331. doi:10.1080/713756761

Barrett, H. C. (2005). Adaptations to predators and prey. In The handbook of evolutionary psychology (pp. 200-223).

Barrett, H. C., \& Behne, T. (2005). Children's understanding of death as the cessation of agency: A test using sleep versus death. Cognition, 96(2), 93-108.

Barth, H., Kanwisher, N., \& Spelke, E. S. (2003). The construction of large number representations in adults. Cognition, 86(3), 201-221. doi:10.1016/ S0010-0277(02)00178-6

Barth, H., La Mont, K., Lipton, J. S., Dehaene, S., Kanwisher, N., \& Spelke, E. S. (2006). Non-symbolic arithmetic in adults and young children. Cognition, 98(3), 199-222. doi:10.1016/j.cognition.2004.09.011

Bateman, P. W., \& Fleming, P. A. (2011). Who are you looking at? Hadeda lbises use direction of gaze, head orientation and approach speed in their risk assessment of a potential predator. Journal of Zoology, 285(4), 316-323.

Bednekoff, P. A., \& Balda, R. P. (1996). Observational spatial memory in Clark's nutcrackers and mexican jays. Animal Behaviour, 52(4), 833-839.

Bednekoff, P. A., Kamil, A. C., \& Balda, R. P. (1997). Clark's nutcracker (aves: Corvidae) spatial memory: Interference effects on cache recovery performance? Ethology, 103(7), 554-565.

Behne, T., Carpenter, M., Call, J., \& Tomasello, M. (2005). Unwilling versus unable: Infants' understanding of intentional action. Developmental Psychology, 41(2), 328-37. doi:10.1037/0012-1649.41.2.328 
Bekoff, M., Allen, C., \& Burghardt G.M. (Eds.). The Cognitive Animal: Empirical and Theoretical Perspectives on Animal Cognition. (2002). Cambridge, MA, US: MIT Press.

Bekoff, M., Townsend, S. E., \& Jamieson, D. (1994). Beyond monkey minds: Toward a richer cognitive ethology. Behavioural and Brain Sciences, 17(3), 571-572.

Beran, M. J. (2001). Summation and numerousness judgments of sequentially presented sets of items by chimpanzees (Pan troglodytes). Journal of Comparative Psychology, 115(2), 181-191.

Beran, M. J. (2004). Chimpanzees (Pan troglodytes) respond to nonvisible sets after one-by-one addition and removal of items. Journal of Comparative Psychology, 118(1), 25-36.

Beran, M. J. (2006). Quantity perception by adult humans (Homo sapiens), chimpanzees (Pan troglodytes), and rhesus macaques (Macaca mulatto) as a function of stimulus organization. International Journal of Comparative Psychology, 19(4), 386-397.

Beran, M. J. (2007). Rhesus monkeys (Macaca mulatta) enumerate large and small sequentially presented sets of items using analog numerical representations. Journal of Experimental Psychology: Animal Behaviour Processes, 33(1), 42-54.

Beran, M. J. (2008). The evolutionary and developmental foundations of mathematics. PLoS Biology, 6(2). doi:10.1371/journal.pbio.0060019

Beran, M. J. (2012). Quantity judgments of auditory and visual stimuli by chimpanzees (pan troglodytes). Journal of Experimental Psychology: Animal Behaviour Processes, 38(1), 23.

Beran, M. J., \& Beran, M. M. (2004). Chimpanzees remember the results of one-byone addition of food items to sets over extended time periods. Psychological Science, 15(2), 94-99.

Beran, M. J., Beran, M. M., \& Menzel, C. R. (2005). Spatial memory and monitoring of hidden items through spatial displacements by chimpanzees (Pan troglodytes). Journal of Comparative Psychology, 119(1), 14-22.

Beran, M. J., Beran, M. M., Harris, E. H., \& Washburn, D. A. (2005). Ordinal judgments and summation of nonvisible sets of food items by two chimpanzees and a rhesus macaque. Journal of Experimental Psychology: Animal Behaviour Processes, 31(3), 351-362.

Beran, M. J., Evans, T. A., \& Hoyle, D. (2011). Numerical judgments by chimpanzees (Pan troglodytes) in a token economy. Journal of Experimental Psychology: Animal Behaviour Processes, 37(2), 165. 
Bird, C. D., \& Emery, N. J. (2010). Rooks perceive support relations similar to sixmonth-old babies. Proceedings of the Royal Society B-Biological Sciences, 277(1678), 147-151. doi:10.1098/rspb.2009.1456

Bisazza, A., Piffer, L., Serena, G., \& Agrillo, C. (2010). Ontogeny of numerical abilities in fish. PLoS One, 5(11). doi:10.1371/journal.pone.0015516

Blackwell, G. L. (2005). Another world: The composition and consequences of the introduced mammal fauna of New Zealand. Australian Zoologist, 33(1), 108-118.

Bluff, L. A., Weir, A. A. S., Rutz, C., Wimpenny, J. H., \& Kacelnik, A. (2007). Toolrelated cognition in New Caledonian crows. Comparative Cognition \& Behaviour Review, 2(1), 1-25.

Bogale, B. A., Kamata, N., Mioko, K., \& Sugita, S. (2011). Quantity discrimination in jungle crows, Corvus macrorhynchos. Animal Behaviour.

Boysen, S. T., \& Berntson, G. G. (1989). Numerical competence in a chimpanzee (Pan troglodytes). Journal of Comparative Psychology, 103(1), 23-31.

Boysen, S. T., \& Berntson, G. G. (1995). Responses to quantity: Perceptual versus cognitive mechanisms in chimpanzees (Pan troglodytes). Journal of Experimental Psychology: Animal Behaviour Processes, 21(1), 82-86.

Bräuer, J., Call, J., \& Tomasello, M. (2004). Visual perspective taking in dogs (Canis familiaris) in the presence of barriers. Applied Animal Behaviour Science, 88, 299-317.

Bräuer, J., Call, J., \& Tomasello, M. (2005). All great ape species follow gaze to distant locations and around barriers. Journal of Comparative Psychology, 119(2), 145-54. doi:10.1037/0735-7036.119.2.145

Brannon, E. M., Wusthoff, C. J., Gallistel, C. R., \& Gibbon, J. (2001). Numerical subtraction in the pigeon: Evidence for a linear subjective number scale. Psychological Science, 12(3), 238-243.

Bugnyar, T. (2007). An integrative approach to the study of 'theory-of-mind'-like abilities in ravens. Japanese Journal of Animal Psychology, 57(1), 1527.

Bugnyar, T., \& Heinrich, B. (2005). Ravens, Corvus corax, differentiate between knowledgeable and ignorant competitors. Proceedings of the Royal Society B: Biological Sciences, 272(1573), 1641.

Bugnyar, T., \& Heinrich, B. (2006). Pilfering ravens, Corvus corax, adjust their behaviour to social context and identity of competitors. Animal Cognition, 9(4), 369-376.

Bugnyar, T., \& Kotrschal, K. (2002). Observational learning and the raiding of food caches in ravens, Corvus corax : Is it "tactical" deception? Animal Behaviour, 64(2), 185-195. 
Bugnyar, T., Stöwe, M., \& Heinrich, B. (2004). Ravens, Corvus corax, follow gaze direction of humans around obstacles. Proceedings of the Royal Society B: Biological Sciences, 271(1546), 1331-1336.

Bugnyar, T. T. (2011). Knower-guesser differentiation in ravens: Others' viewpoints matter. Proceedings of the Royal Society B: Biological Sciences, 278(1705).

Burger, J., Gochfeld, M., \& Murray, B. G. (1992). Risk discrimination of eye contact and directness of approach in black iguanas (Ctenosaura similis). Journal of Comparative Psychology, 106(1), 97.

Burns, K. C. (2009). Fine-scale food hoarding decisions in New Zealand robins (Petroica australis): Is inter-sexual competition important? Journal of Ornithology, 150(2), 321-328-321-328.

Burns, K. C., \& Steer, J. (2006). Dominance rank influences food hoarding in New Zealand robins petroica australis. Ibis, 148(2), 266-272.

Burns, K. C., \& Van Horik, J. (2007). Sexual differences in food re-caching by New Zealand robins petroica australis. Journal of Avian Biology, 38(3), 394-398.

Call, J. (2007). Apes know that hidden objects can affect the orientation of other objects. Cognition, 105(1), 1-25. doi:10.1016/j.cognition.2006.08.004

Call, J., \& Tomasello, M. (1999). A nonverbal false belief task: The performance of children and great apes. Child Development, 70(2), 381-395.

Call, J., Hare, B., Carpenter, M., \& Tomasello, M. (2004). 'Unwilling' versus 'unable': Chimpanzees' understanding of human intentional action. Developmental Science, 7(4), 488-498. doi:10.1111/j.1467-7687.2004.00368.x

Cantlon, J. F., \& Brannon, E. M. (2006). The effect of heterogeneity on numerical ordering in rhesus monkeys. Infancy, 9(2), 173-189.

Cantlon, J. F., Platt, M. L., \& Brannon, E. M. (2009). Beyond the number domain. Trends in Cognitive Sciences, 13(2), 83-91-83-91.

Carazo, P., Font, E., Forteza-Behrendt, E., \& Desfilis, E. (2009). Quantity discrimination in Tenebrio molitor: Evidence of numerosity discrimination in an invertebrate? Animal Cognition, 12(3), 463-470.

Carey, S. (1985). Conceptual change in childhood (p. 240). Cambridge, Mass.: MIT Press.

Carey, S. (1998). Knowledge of number: Its evolution and ontogeny. Science, 282(5389), 641-642.

Carey, S., \& Williams, T. (2001). The role of object recognition in young infants' object segregation. Journal of Experimental Child Psychology, 78(1), 55-60. 
Carter, J., Lyons, N. J., Cole, H. L., \& Goldsmith, A. R. (2008). Subtle cues of predation risk: Starlings respond to a predator's direction of eye-gaze. Proceedings of the Royal Society B: Biological Sciences, 275(1644), 1709-1715.

Changeux, J. P., \& Dehaene, S. (1989). Neuronal models of cognitive functions. Cognition, 33, 63-109.

Cheng, K. (1986). A purely geometric module in the rat's spatial representation. Cognition, 23(2), 149-178.

Cheries, E. W., Mitroff, S. R., Wynn, K., \& Scholl, B. J. (2008). Cohesion as a constraint on object persistence in infancy. Developmental Science, 11(3), 427-432.

Chiandetti, C., \& Vallortigara, G. (2011). Intuitive physical reasoning about occluded objects by inexperienced chicks. Proceedings of the Royal Society B: Biological Sciences, 278(1718), 2621-7. doi:10.1098/rspb.2010.2381

Chiesa, A. D., Speranza, M., Tommasi, L., \& Vallortigara, G. (2006). Spatial cognition based on geometry and landmarks in the domestic chick (Gallus gallus). Behavioural Brain Research, 175(1), 119-127.

Church, R. M., \& Broadbent, H. A. (1990). Alternative representations of time, number, and rate. Cognition, 37, 55-81.

Clara, E., Regolin, L., Vallortigara, G., \& Rogers, L. J. (2009). Chicks prefer to peck at insect-like elongated stimuli moving in a direction orthogonal to their longer axis. Animal Cognition, 12(6), 755-765.

Clayton, N. S. (1994). The role of age and experience in the behavioural development of food-storing and retrieval in marsh tits, Parus palustris. Animal Behaviour, 47(6), 1435-1444.

Clayton, N. S. (1995). Development of memory and the hippocampus: Comparison of food-storing and non-storing birds on a one-trial associative memory task. Journal of Neuroscience, 15(4), 2796-2807.

Clayton, N. S., \& Dickinson, A. (1998). Episodic-like memory during cache recovery by scrub jays. Nature, 395(6699), 272-274.

Clayton, N. S., \& Dickinson, A. (1999). Scrub jays (Aphelocoma coerulescens) remember the relative time of caching as well as the location and content of their caches. Journal of Comparative Psychology, 113(4), 403-416.

Clayton, N. S., Dally, J. M., \& Emery, N. J. (2007). Social cognition by food-caching corvids: The western scrub-jay as a natural psychologist. Philosophical Transactions of the Royal Society B: Biological Sciences, 362(1480), 507-522. 
Clayton, N. S., Dally, J. M., Gilbert, J., \& Dickinson, A. (2005). Food caching by western scrub-jays (Aphelocoma californica) is sensitive to the conditions at recovery. Journal of Experimental Psychology: Animal Behaviour Processes, 31(2), 115-124.

Clayton, N. S., Emery, N. J., \& Dickinson, A. (2006a). The prospective cognition of food caching and recovery by western scrub-jays (Aphelocoma californica). Comparative Cognition \& Behaviour Review, 1(1), 1-11.

Clayton, N. S., Emery, N. J., \& Dickinson, A. (2006b). The rationality of animal memory: Complex caching strategies of western scrub jays. In S. L. Hurley \& M. Nudds (Eds.), Rational animals? (pp. 197-216). New York, NY, US: Oxford University Press. viii.

Clayton, N. S., Yu, K. S., \& Dickinson, A. (2003). Interacting cache memories: Evidence for flexible memory use by western scrub-jays (Aphelocoma californica). Journal of Experimental Psychology: Animal Behaviour Processes, 29(1), 14-22.

Clearfield, M. W., \& Westfahl, S. M. C. (2006). Familiarization in infants' perception of addition problems. Journal of Cognition and Development, 7(1), 27-43.

Cohen, L. B., \& Marks, K. S. (2002). How infants process addition and subtraction events. Developmental Science, 5(2), 186-201.

Cook, R. G. (2000). The comparative psychology of avian visual cognition. Current Directions in Psychological Science, 9(3), 83-89.

Cordes, S., Gelman, R., Gallistel, C. R., \& Whalen, J. (2001). Variability signatures distinguish verbal from nonverbal counting for both large and small numbers. Psychonomic Bulletin \& Review, 8(4), 698-707.

Dadda, M., Piffer, L., Agrillo, C., \& Bisazza, A. (2009). Spontaneous number representation in mosquitofish. Cognition, 112(2), 343-348.

Dally, J. M., Clayton, N. S., \& Emery, N. J. (2006). The behaviour and evolution of cache protection and pilferage. Animal Behaviour, 72(1), 13-23.

Dally, J. M., Emery, N. J., \& Clayton, N. S. (2005). Cache protection strategies by western scrub-jays, Aphelocoma californica: Implications for social cognition. Animal Behaviour, 70(6), 1251-1263.

Daugherty, C. H., Gibbs, G. W., \& Hitchmough, R. A. (1993). Mega-island or microcontinent? New Zealand and its fauna. Trends in Ecology \& Evolution, 8(12), 437-442.

Davis, H., \& Pérusse, R. (1988). Numerical competence in animals: Definitional issues, current evidence, and a new research agenda. Behavioural and Brain Sciences, 11(04), 561-579. 
Dehaene, S., Dehaene-Lambertz, G., \& Cohen, L. (1998). Abstract representations of numbers in the animal and human brain. Trends in Neurosciences, 21(8), 355-361.

Dehaene, S., Izard, V., Spelke, E. S., \& Pica, P. (2008). Log or linear? Distinct intuitions of the number scale in western and Amazonian indigene cultures. Science, 320(5880), 1217-1217.

Dittrich, W. H., \& Lea, S. E. G. (1993). Motion as a natural category for pigeons: Generalization and a feature-positive effect. Journal of the Experimental Analysis of Behaviour, 59(1), 115.

Dittrich, W. H., Lea, S., Barrett, J., \& Gurr, P. (1998). Categorization of natural movements by pigeons: Visual concept discrimination and biological motion. Journal of the Experimental Analysis of Behaviour, 70(3), 281.

Dunlap, A. S., Chen, B. B., Bednekoff, P. A., Greene, T. M., \& Balda, R. P. (2006). A state-dependent sex difference in spatial memory in pinyon jays, Gymnorhinus cyanocephalus: Mated females forget as predicted by natural history. Animal Behaviour, 72(2), 401-411.

Emery, N. J. (2000). The eyes have it: The neuroethology, function and evolution of social gaze. Neuroscience and Biobehavioural Reviews, 24(6), 581-604. doi: 10.1016/S0149-7634(00)00025-7

Emery, N. J., \& Clayton, N. S. (2004). Comparing the complex cognition of birds and primates. In L. J. Rogers \& G. Kaplan (Eds.), Comparative vertebrate cognition (pp. 3-55). Springer.

Emery, N. J., \& Clayton, N. S. (2008). How to build a scrub-jay that reads minds. In, Origins of the social mind: Evolutionary and developmental views (S. Itakura \& K. Fujita, Eds.). 211-211. Tokyo, Japan: Springer.

Emery, N. J., \& Clayton, N. S. (2009). Comparative social cognition. Annual Review of Psychology, 60, 87-113.

Emery, N. J., Clayton, N. S., Bolhuis, J. J., \& Giraldeau, L. A. (2005). Animal cognition. In The behaviour of animals: Mechanisms, function, and evolution (pp. 515-515). Malden, MA, USA: Wiley-Blackwell.

Emery, N. J., Dally, J. M., \& Clayton, N. S. (2004). Western scrub-jays (Aphelocoma californica) use cognitive strategies to protect their caches from thieving conspecifics. Animal Cognition, 7(1), 37-43.

Emmerton, J. (1998). Numerosity differences and effects of stimulus density on pigeons' discrimination performance. Animal Learning \& Behaviour, 26(3), 243-256.

Farnsworth, G. L., \& Smolinski, J. L. (2006). Numerical discrimination by wild northern mockingbirds. The Condor, 108(4), 953-953. 
Feigenson, L., \& Carey, S. (2003). Tracking individuals via object-files: Evidence from infants' manual search. Developmental Science, 6(5), 568-584.

Feigenson, L., \& Carey, S. (2005). On the limits of infants' quantification of small object arrays. Cognition, 97(3), 295-313.

Feigenson, L., Carey, S., \& Hauser, M. D. (2002). The representations underlying infants' choice of more: Object files versus analog magnitudes. Psychological Science, 13(2), 150-156.

Feigenson, L., Dehaene, S., \& Spelke, E. S. (2004). Origins and endpoints of the core systems of number: Reply to Fias and Verguts. Trends in Cognitive Sciences, 8(10), 448-449.

Flack, J.A.D., (1976). The use of frontal spot and crown feathers in inter- and intraspecific display by the South Island robin, Petroica australis australis. Notornis, 23(2), 90-105.

Fleming, C. A. (1950). New Zealand flycatchers of the genus Petroica swainson (aves), parts I and II. In Transactions of the royal society of New Zealand (Vol. 78, pp. 14-47, 126-160).

Flombaum, J. I., \& Santos, L. R. (2005). Rhesus monkeys attribute perceptions to others. Current Biology, 15(5), 447-452.

Flombaum, J. I., Junge, J. A., \& Hauser, M. D. (2005). Rhesus monkeys (Macaca mulatta) spontaneously compute addition operations over large numbers. Cognition, 97(3), 315-325.

Flombaum, J. I., Santos, L. R., \& Hauser, M. D. (2002). Neuroecology and psychological modularity. Trends in Cognitive Sciences, 6(3), 106-108.

Forstmeier, W., Segelbacher, G., Mueller, J. C., \& Kempenaers, B. (2007). Genetic variation and differentiation in captive and wild zebra finches (Taeniopygia guttata). Molecular Ecology, 16(19), 4039-50. doi:10.1111/j.1365-294X. 2007.03444.x

Gallistel, C. R. (1990). The organization of learning learning, development, and conceptual change. Cambridge, MA, US: The MIT Press.

Gallistel, C. R., \& Gelman, R. (1992). Preverbal and verbal counting and computation. Cognition, 44, 43-74. doi:10.1016/0010-0277(92)90050-R

Gallistel, C. R., \& Gelman, R. (2000). Non-verbal numerical cognition: From reals to integers. Trends in Cognitive Sciences, 4(2), 59-65.

Gallistel, C. R., Gelman, R., Holyoak, K. J., \& Morrison, R. G. (2005). Mathematical cognition. In The cambridge handbook of thinking and reasoning. 559-588. New York, NY, US: Cambridge University Press. 
Garland, A., Low, J., \& Burns, K. (2012). Large quantity discrimination by North Island robins (Petroica longipes). Animal Cognition, 1-12. doi:10.1007/ s10071-012-0537-3

Gelman, R., \& Spelke, E. (1981). The development of thoughts about animate and inanimate objects: Implications for research on social cognition. Social Cognitive Development: Frontiers and Possible Futures, 43-66.

Gelman, S. A., \& Gottfried, G. M. (1996). Children's causal explanations of animate and inanimate motion. Child Development, 67(5), 1970-1987.

Gelman, S. A., \& Opfer, J. E. (2002). Development of the animate-inanimate distinction. In Blackwell handbook of childhood cognitive development (pp. 151-166). Wiley Online Library.

Gergely, G., \& Csibra, G. (2003). Teleological reasoning in infancy: The naïve theory of rational action. Trends in Cognitive Sciences, 7(7), 287-292.

Gibbs, G. W. (1998). New Zealand weta. Auckland: Reed Publishing.

Gilby, A. J., Mainwaring, M. C., Rollins, L. A., \& Griffith, S. C. (2011). Parental care in wild and captive zebra finches: Measuring food delivery to quantify parental effort. Animal Behaviour, 81(1), 289-295.

Giret, N., Miklósi, \& Kreutzer, M. (2009). Use of experimenter-given cues by african gray parrots (Psittacus erithacus). Animal Cognition.

Gobbini, M. I., Gentili, C., Ricciardi, E., Bellucci, C., Salvini, P., Laschi, C., \& Pietrini, P. (2011). Distinct neural systems involved in agency and animacy detection. Journal of Cognitive Neuroscience, 23(8), 1911-1920.

Gould, K. L., Ort, A. J., \& Kamil, A. C. (2012). Do Clarkí s nutcrackers demonstrate what-where-when memory on a cache-recovery task? Animal Cognition, 1-8.

Güntürkün, O. (1999). Avian physiology: Vision. In G. C. Whittow (Ed.), Sturkie's avian physiology (5th ed.). San Diego: Academic Press.

Hampton, R. R. (1994). Sensitivity to information specifying the line of gaze of humans in sparrows (Passer domesticus). Behaviour, 130(1/2), 41-51.

Hanus, D., \& Call, J. (2007). Discrete quantity judgments in the great apes (Pan paniscus, Pan troglodytes, Gorilla gorilla, Pongo pygmaeus): The effect of presenting whole sets versus item-by-item. Journal of Comparative Psychology, 121(3), 241-249.

Hare, B., \& Tomasello, M. (1999). Domestic dogs (Canis familiaris) use human and conspecific social cues to locate hidden food. Journal of Comparative Psychology, 113(2), 173-177. 
Hare, B., Brown, M., Williamson, C., \& Tomasello, M. (2002). The domestication of social cognition in dogs. Science (New York, N.Y.), 298(5598), 1634-6. doi: 10.1126/science.1072702

Hare, B., Call, J., \& Tomasello, M. (2001). Do chimpanzees know what conspecifics know? Animal Behaviour, 61(1), 139-151.

Hare, B., Call, J., Agnetta, B., \& Tomasello, M. (2000). Chimpanzees know what conspecifics do and do not see. Animal Behaviour, 59(4), 771-785.

Hauser, M. D. (2000). Homologies for numerical memory span? Trends in Cognitive Sciences, 4(4), 127-128.

Hauser, M. D., MacNeilage, P., \& Ware, M. (1996). Numerical representations in primates. Proceedings of the National Academy of Sciences of the United States of America, 93(4), 1514-1517. doi:10.2307/38613

Hauser, M. D., Spelke, E. S., \& Gazzaniga, M. S. (2004). Evolutionary and developmental foundations of human knowledge: A case study of mathematics. In The cognitive neurosciences (pp. 853-864). Cambridge, MA, US: MIT Press.

Hauser, M. D., Tsao, F., Garcia, P., \& Spelke, E. S. (2003). Evolutionary foundations of number: Spontaneous representation of numerical magnitudes by cottontop tamarins. Proceedings of the Royal Society B: Biological Sciences, 270(1523), 1441-1446. doi:10.1098/rspb.2003.2414

Helme, A. E., Call, J., Clayton, N. S., \& Emery, N. J. (2006). What do bonobos (Pan paniscus) understand about physical contact? Journal of Comparative Psychology, 120(3), 294-302.

Hermer, L., \& Spelke, E. S. (1994). A geometric process for spatial reorientation in young children. Nature, 370(6484), 57-59.

Hermer, L., \& Spelke, E. S. (1996). Modularity and development: The case of spatial reorientation. Cognition, 61(3), 195-232.

Hitchcock, C. L., \& Sherry, D. F. (1990). Long-term memory for cache sites in the black-capped chickadee. Animal Behaviour, 40(4), 701-712.

Holdaway, R. N., Worthy, T. H., \& Tennyson, A. J. D. (2001). A working list of breeding bird species of the New Zealand region at first human contact. New Zealand Journal of Zoology, 28(2), 119-187.

Hollard, V. D., \& Delius, J. D. (1982). Rotational invariance in visual pattern recognition by pigeons and humans. Science, 218(4574), 804-806.

Honig, W. K., \& Matheson, W. R. (1995). Discrimination of relative numerosity and stimulus mixture by pigeons with comparable tasks. Journal of Experimental Psychology: Animal Behaviour Processes, 21(4), 348-363. 
Honig, W. K., \& Stewart, K. E. (1988). Pigeons can discriminate locations presented in pictures. Journal of the Experimental Analysis of Behaviour, 50(3), 541-551.

Honig, W. K., \& Stewart, K. E. (1989). Discrimination of relative numerosity by pigeons. Animal Learning \& Behaviour, 17(2), 134-146.

Hood, B. M., Hauser, M. D., Anderson, L., \& Santos, L. (1999). Gravity biases in a non-human primate? Developmental Science, 2(1), 35-41.

Horner, V., \& Whiten, A. (2005). Causal knowledge and imitation/emulation switching in chimpanzees (Pan troglodytes) and children (Homo sapiens). Animal Cognition, 8(3), 164-81. doi:10.1007/s10071-004-0239-6

Hunt, G. R., Rutledge, R. B., \& Gray, R. D. (2006). The right tool for the job: What strategies do wild New Caledonian crows use? Animal Cognition, 9(4), 307-316. doi:10.1007/s10071-006-0047-2

Hunt, S., Low, J., \& Burns, K. C. (2008). Adaptive numerical competency in a foodhoarding songbird. Proceedings of the Royal Society B: Biological Sciences, 275(1649), 2373-2379. doi:10.1098/rspb.2008.0702

Irie-Sugimoto, N., Kobayashi, T., Sato, T., \& Hasegawa, T. (2009). Relative quantity judgment by Asian elephants (Elephas maximus). Animal Cognition, 12(1), 193-199.

Izard, V., \& Dehaene, S. (2008). Calibrating the mental number line. Cognition, 106(3), 1221-1247.

Jaakkola, K., Fellner, W., Erb, L., Rodriguez, M., \& Guarino, E. (2005). Understanding of the concept of numerically "less" by bottlenose dolphins (Tursiops truncatus). Journal of Comparative Psychology, 119(3), 296-303.

Jaime, M., Lopez, J. P., \& Lickliter, R. (2009). Bobwhite quail (Colinus virginianus) hatchlings track the direction of human gaze. Animal Cognition, 12(4), 559-565.

Jewell, T., \& Brock, P. D. (2002). A review of the New Zealand stick insects: New genera and synonymy, keys, and a catalogue. Journal of Orthoptera Research, 11(2), 189-197. doi:http://dx.doi.org/10.1665/1082-6467

Johansson, G. (1973). Visual perception of biological motion and a model for its analysis. Attention, Perception, \& Psychophysics, 14(2), 201-211.

Johnson, S. C., Booth, A., \& O'Hearn, K. (2001). Inferring the goals of a nonhuman agent. Cognitive Development, 16(1), 637-656.

Jones, R. B. (1980). Reactions of male domestic chicks to two-dimensional eye-like shapes. Animal Behaviour, 28(1), 212-218. 
Jordan, K. E., \& Brannon, E. M. (2006). A common representational system governed by weber's law: Nonverbal numerical similarity judgments in 6-yearolds and rhesus macaques. Journal of Experimental Child Psychology, 95(3), 215-229.

Kaminski, J., Riedel, J., Call, J., \& Tomasello, M. (2005). Domestic goats, Capra hircus, follow gaze direction and use social cues in an object choice task. Animal Behaviour, 69(1), 11-18.

Kaneko, T., \& Tomonaga, M. (2011). The perception of self-agency in chimpanzees (Pan troglodytes). Proceedings of the Royal Society B: Biological Sciences, 278(1725), 3694-702. doi:10.1098/rspb.2011.0611

Kilian, A., von Fersen, L., \& Güntürkün, O. (2005). Left hemispheric advantage for numerical abilities in the bottlenose dolphin. Behavioural Processes, 68(2), 179-184.

Kilian, A., Yaman, S., von Fersen, L., \& Güntürkün, O. (2003). A bottlenose dolphin discriminates visual stimuli differing in numerosity. Learning \& Behaviour, 31(2), 133-142.

Koehler, O. (1941). Vom erlernen unbenannter Anzahlen bei Vögeln / The learning of unnamed amounts in birds. Naturwissenschaften, 29, 201-218.

Krachun, C., Carpenter, M., Call, J., \& Tomasello, M. (2009). A competitive nonverbal false belief task for children and apes. Developmental Science, 12(4), 521-535.

Krusche, P., Uller, C., \& Dicke, U. (2010). Quantity discrimination in salamanders. Journal of Experimental Biology, 213(11), 1822-1828. doi:10.1242/jeb.039297

Kundey, S. M., De Los Reyes, A., Taglang, C., Baruch, A., \& German, R. (2010). Domesticated dogs' (Canis familiaris) use of the solidity principle. Animal Cognition, 13(3), 497-505. doi:10.1007/s10071-009-0300-6

Lee, K. E., \& Zelanda, N. (1959). The earthworm fauna of New Zealand. Department of Scientific and Industrial Research Wellington, New Zealand.

Leslie, A. M. (1994). ToMM, toby, and agency: Core architecture and domain specificity. In Mapping the mind: Domain specificity in cognition and culture (pp. 119-148).

Leslie, A. M., \& Keeble, S. (1987). Do six-month-old infants perceive causality? Cognition, 25(3), 265-288.

Lipton, J. S., \& Spelke, E. S. (2003). Origins of number sense: Large-number discrimination in human infants. Psychological Science, 14(5), 396-401.

Low, J. (2010). Preschoolers' implicit and explicit false-belief understanding: Relations with complex syntactical mastery. Child Development, 81(2), 597-615. doi:10.1111/j.1467-8624.2009.01418.x 
Low, J., Burns, K. C., \& Hauber, M. E. (2009). Wild number sense in brood parasitic brown-headed cowbirds. Ibis, 151(4), 775-777-775-777.

Lyon, B. E. (2003). Egg recognition and counting reduce costs of avian conspecific brood parasitism. Nature, 422(6931), 495-499.

Maloney, R. F., \& McLean, I. G. (1995). Historical and experimental learned predator recognition in free-living New Zealand robins. Animal Behaviour, 50(5), 1193-1201. doi:10.1016/0003-3472(95)80036-0

Maros, K., Gácsi, M., \& Miklósi, A. (2008). Comprehension of human pointing gestures in horses (Equus caballus). Animal Cognition, 11(3), 457-66. doi: 10.1007/s10071-008-0136-5

Marzluff, J. M., Walls, J., Cornell, H. N., Withey, J. C., \& Craig, D. P. (2010). Lasting recognition of threatening people by wild American crows. Animal Behaviour, 79(3), 699-707.

Mascalzoni, E., Regolin, L., \& Vallortigara, G. (2010). Innate sensitivity for selfpropelled causal agency in newly hatched chicks. Proceedings of the National Academy of Sciences, 107(9), 4483-4485.

McComb, K., Packer, C., \& Pusey, A. (1994). Roaring and numerical assessment in contests between groups of female lions, Panthera leo. Animal Behaviour, 47(2), 379-387. doi:10.1006/anbe.1994.1052

McCrink, K., \& Wynn, K. (2004). Large-number addition and subtraction by 9-monthold infants. Psychological Science, 15(11), 776-781.

McGavin, S. (2009). Density and pair fidelity in a translocated population of North Island robins (Petroica longipes). Notornis, 56, 206-212.

Meck, W. H., \& Church, R. M. (1983). A mode control model of counting and timing processes. Journal of Experimental Psychology: Animal Behaviour Processes, 9(3), 320-334.

Meck, W. H., Church, R. M., \& Gibbon, J. (1985). Temporal integration in duration and number discrimination. Journal of Experimental Psychology: Animal Behaviour Processes, 11(4), 591-597.

Meltzoff, A. N. (2007). 'Like me': A foundation for social cognition. Developmental Science, 10(1), 126-134.

Meltzoff, A. N., \& Moore, M. K. (1977). Imitation of facial and manual gestures by human neonates. Science, 198(4312), 75-78.

Menzies, I. J., \& Burns, K. C. (2008). Food hoarding in the New Zealand robin: A review and synthesis. In E. A. Weber \& L. H. Krause (Eds.), Animal behaviour: New research. New York, NY, US: Nova Science Publishers. 
Menzies, I. J., \& Burns, K. C. (2010). Temporal shifts in the pair-bond dynamics of New Zealand robins (Petroica australis). New Zealand Journal of Ecology, $34(2)$.

Mewaldt, L. R. (1956). Nesting behaviour of the Clark's nutcracker. The Condor, 58(1), 3-23.

Miklósi, A., Polgárdi, R., Topál, J., \& Csányi, V. (1998). Use of experimenter-given cues in dogs. Animal Cognition, 1(2), 113-121.

Miklósi, A., Kubinyi, E., Topal, J., Gacsi, M., Viranyi, Z., \& Csanyi, V. (2003). A simple reason for a big difference: Wolves do not look back at humans, but dogs do. Current Biology, 13(9), 763-766. doi:10.1016/S0960-9822(03)00263-X

Miller, H. C., \& Lambert, D. M. (2006). A molecular phylogeny of New Zealand's Petroica (aves: Petroicidae) species based on mitochondrial DNA sequences. Molecular Phylogenetics and Evolution, 40(3), 844-855. doi:10.1016/j.ympev. 2006.04.012

Miller, H. C., Rayburn-Reeves, R., \& Zentall, T. R. (2009). What do dogs know about hidden objects? Behavioral Processes, 81(3), 439-46. doi:10.1016/j.beproc. 2009.03.018

Moreby, S. J., Aebischer, N. J., \& Southway, S. (2006). Food preferences of grey partridge chicks, Perdix perdix, in relation to size, colour and movement of insect prey. Animal Behaviour, 71(4), 871-878.

Myowa-Yamakoshi, M., Scola, C., \& Hirata, S. (2012). Humans and chimpanzees attend differently to goal-directed actions. Nature Communications, 3, 693. doi:doi:10.1038/ncomms1695

O'Connell, S., \& Dunbar, R. I. M. (2005). The perception of causality in chimpanzees (Pan spp.). Animal Cognition, 8(1), 60-66.

Pack, A. A., \& Herman, L. M. (2004). Bottlenosed dolphins (Tursiops truncatus) comprehend the referent of both static and dynamic human gazing and pointing in an object-choice task. Journal of Comparative Psychology, 118(2), 160-171.

Pattison, K. F., Miller, H. C., Rayburn-Reeves, R., \& Zentall, T. (2010). The case of the disappearing bone: Dogs' understanding of the physical properties of objects. Behavioral Processes, 85(3), 278-82. doi:10.1016/j.beproc. 2010.06.016

Penn, D. C., \& Povinelli, D. J. (2007). On the lack of evidence that non-human animals possess anything remotely resembling a 'Theory of Mind'. Philosophical Transactions of the Royal Society of London Series B, Biological Sciences, 362(1480), 731-744. doi:10.1098/rstb.2006.2023 
Penn, D. C., Holyoak, K. J., \& Povinelli, D. J. (2008). Darwin's mistake: Explaining the discontinuity between human and nonhuman minds. Behavioural and Brain Sciences, 31(2), 109-130.

Pepperberg, I. M. (2006). Grey parrot numerical competence: A review. Animal Cognition, 9(4), 377-391.

Pepperberg, I. M., Hurley, S. L., \& Chater, N. (2005). Insights into vocal imitation in african grey parrots (Psittacus erithacus). In Perspectives on imitation: From neuroscience to social science (Vol. 1, pp. 243-262). Cambridge, MA, US: MIT Press.

Perdue, B. M., Talbot, C. F., Stone, A. M., \& Beran, M. J. (2012). Putting the elephant back in the herd: Elephant relative quantity judgments match those of other species. Animal Cognition, 1-7.

Peron, F., Chardard, C., Nagle, L., \& Bovet, D. (2011). Do African grey parrots (Psittacus erithacus) know what a human experimenter does and does not see? Behavioural Processes, 87(2), 237-240.

Peron, F., Rat-Fischer, L., Nagle, L., \& Bovet, D. (2010). 'Unwilling' versus 'unable' do grey parrots understand human intentional actions? Interaction Studies, 11(3), 428-441. doi:10.1075/is.11.3.06per

Phillips, W., Barnes, J. L., Mahajan, N., Yamaguchi, M., \& Santos, L. R. (2009). 'Unwilling' versus 'unable': Capuchin monkeys (Cebus apella) understanding of human intentional action. Developmental Science, 12(6), 938-945.

Pika, S., \& Bugnyar, T. (2011). The use of referential gestures in ravens (Corvus corax) in the wild. Nature Communications, 2, 560. doi:10.1038/ncomms1567

Pollok, B., Prior, H., \& Güntürkün, O. (2000). Development of object permanence in food-storing magpies (Pica pica). Journal of Comparative Psychology, 114(2), 148-157. doi:10.1037//0735-7036.114.2.148

Povinelli, D. J. (2000). Folk physics for apes: The chimpanzee's theory of how the world works. Oxford ; New York: Oxford University Press.

Povinelli, D. J., \& Vonk, J. (2003). Chimpanzee minds: Suspiciously human? Trends in Cognitive Sciences, 7(4), 157-160. doi:10.1016/S1364-6613(03)00053-6

Povinelli, D. J., \& Vonk, J. (2004). We don't need a microscope to explore the chimpanzee's mind. Mind \& Language, 19(1), 1-28. doi:10.1111/j. 1468-0017.2004.00244.x

Povinelli, D. J., Perilloux, H. K., Reaux, J. E., \& Bierschwale, D. T. (1998). Young and juvenile chimpanzees' (Pan troglodytes) reactions to intentional versus accidental and inadvertent actions. Behavioural Processes, 42(2-3), 205-218.

Powlesland, R. G. (1980). Food-Storing behaviour of the South Island robin. Mauri Ora, 8, 11-20. 
Powlesland, R. G. (1981). The foraging behaviour of the South Island robin. Notornis, 28, 89-102.

Proops, L., \& McComb, K. (2010). Attributing attention: The use of human-given cues by domestic horses (Equus caballus). Animal Cognition, 13(2), 197-205.

Rakison, D. H., \& Poulin-Dubois, D. (2001). Developmental origin of the animateinanimate distinction. Psychological Bulletin, 127(2), 209.

Range, F., Horn, L., Bugnyar, T., Gajdon, G. K., \& Huber, L. (2009). Social attention in keas, dogs, and human children. Animal Cognition, 12(1), 181-192.

Regolin, L., Vallortigara, G., \& Zanforlin, M. (1995). Object and spatial representations in detour problems by chicks. Animal Behaviour, 49(1), 195-199.

Rizzolatti, G., Fogassi, L., \& Gallese, V. (2008). Mirrors in the mind. In M. H. Immordino-Yang (Ed.), The jossey-bass reader on the brain and learning (pp. 12-19). San Francisco, CA, US: Jossey-Bass.

Roberts, W. A., Coughlin, R., \& Roberts, S. (2000). Pigeons flexibly time or count on cue. Psychological Science, 11(3), 218-222.

Rosa-Salva, O., Regolin, L., \& Vallortigara, G. (2010). Faces are special for newly hatched chicks: Evidence for inborn domain-specific mechanisms underlying spontaneous preferences for face-like stimuli. Developmental Science, 13(4), 565-577. doi:10.1111/j.1467-7687.2009.00914.x

Rugani, R., Fontanari, L., Simoni, E., Regolin, L., \& Vallortigara, G. (2009). Arithmetic in newborn chicks. Proceedings of the Royal Society B: Biological Sciences, 276(1666), 2451-2451.

Rugani, R., Regolin, L., \& Vallortigara, G. (2008). Discrimination of small numerosities in young chicks. Journal of Experimental Psychology: Animal Behaviour Processes, 34(3), 388-399.

Salva, O. R., Farroni, T., Regolin, L., Vallortigara, G., \& Johnson, M. H. (2011). The evolution of social orienting: Evidence from chicks (Gallus gallus) and human newborns. PLOS ONE, 6(4), e18802. doi:10.1371/journal.pone.0018802

Sanford, K., \& Clayton, N. S. (2008). Motivation and memory in zebra finch (Taeniopygia guttata) foraging behaviour. Animal Cognition, 11(2), 189-198.

Santos, L. R. (2004). 'Core knowledges': A dissociation between spatiotemporal knowledge and contact-mechanics in a non-human primate? Developmental Science, 7(2), 167-174.

Santos, L. R., \& Hauser, M. D. (1999). How monkeys see the eyes: Cotton-top tamarins' reaction to changes in visual attention and action. Animal Cognition, 2(3), 131-139. 
Santos, L. R., Barnes, J. L., \& Mahajan, N. (2005). Expectations about numerical events in four lemur species (Eulemur fulvus, Eulemur mongoz, Lemur catta and Varecia rubra). Animal Cognition, 8(4), 253-262-253-262.

Santos, L. R., Sulkowski, G. M., Spaepen, G. M., \& Hauser, M. D. (2002). Object individuation using property/kind information in rhesus macaques (Macaca mulatta). Cognition, 83(3), 241-264.

Scarf, D. Colombo, M. (2008). Representation of serial order: A comparative analysis of humans, monkeys, and pigeons. Brain Research Bulletin, 76(3), 307-312.

Scarf, D., Danly, E., Morgan, G., Colombo, M., \& Terrace, H.S. (2011). Sequential planning in rhesus monkeys (Macaca mulatta). Animal Cognition, 14(3), 317-324.

Scarf, D., Hayne, H., \& Colombo, M. (2011). Pigeons on par with primates in numerical competence. Science, 334(6063), 1664-1664.

Scheid, C., \& Bugnyar, T. (2008). Short-term observational spatial memory in jackdaws (Corvus monedula) and ravens (Corvus corax). Animal Cognition, 11(4), 691-698.

Schloegl, C., Kotrschal, K., \& Bugnyar, T. (2007). Gaze following in common ravens, Corvus corax: Ontogeny and habituation. Animal Behaviour, 74(4), 769-778.

Scholl, B. J., \& Tremoulet, P. D. (2000). Perceptual causality and animacy. Trends in Cognitive Sciences, 4(8), 299-309.

Sherry, D. F. (1985). Food storage by birds and mammals. Adv. Study Behav, 15(153), 88.

Shettleworth, S. J. (2009). Animal cognition: Deconstructing avian insight. Current Biology, 19(22), R1039-R1040. doi:10.1016/j.cub.2009.10.022

Siegler, R. S., \& Liebert, R. M. (1975). Acquisition of formal scientific reasoning by 10-and 13-year-olds: Designing a factorial experiment. Developmental Psychology, 11(3), 401.

Simon, T. J., Hespos, S. J., \& Rochat, P. (1995). Do infants understand simple arithmetic? A replication of wynn (1992). Cognitive Development, 10(2), 253-269.

Slabbekoorn, H., \& Ripmeester, E. A. P. (2007). Birdsong and anthropogenic noise: Implications and applications for conservation. Molecular Ecology, 17(1), 72-83.

Slater, A. M., Bremner, J. G., Johnson, S. P., \& Hayes, R. A. (2010). The role of perceptual and cognitive processes in addition--subtraction studies with 5month-old infants. Infant Behaviour and Development, 33(4), 685-688. 
Slaughter, V., Jaakkola, R., \& Carey, S. (1999). Constructing a coherent theory: Children's biological understanding of life and death. In Children's understanding of biology and health.

Smith, B. P., \& Litchfield, C. A. (2010). Dingoes (Canis dingo) can use human social cues to locate hidden food. Animal Cognition, 13(2), 367-376. doi:10.1007/ s10071-009-0287-z

Sol, D., \& Lefebvre, L. (2000). Behavioural flexibility predicts invasion success in birds introduced to New Zealand. Oikos, 90(3), 599-605.

Spelke, E. S. (1990). Principles of object perception. Cognitive Science, 14(1), 29-56.

Spelke, E. S. (2000). Core knowledge. American Psychologist, 55(11), 1233-1243.

Spelke, E. S., \& Kinzler, K. D. (2007). Core knowledge. Developmental Science, 10(1), 89-89.

Spelke, E. S., Breinlinger, K., Macomber, J., \& Jacobson, K. (1992). Origins of knowledge. Psychological Review, 99(4), 605-632.

Spelke, E. S., Gentner, D., \& Goldin-Meadow, S. (2003). What makes us smart? Core knowledge and natural language. In Language in mind: Advances in the study of language and thought (pp. 277-311). Cambridge, MA, US: MIT Press.

Steer, J., \& Burns, K. C. (2008). Seasonal variation in male-female competition, cooperation and selfish hoarding in a monogamous songbird. Behavioural Ecology and Sociobiology, 62(7), 1175-1183.

Sulkowski, G. M., \& Hauser, M. D. (2001). Can rhesus monkeys spontaneously subtract? Cognition, 79(3), 239-262.

Taylor, A. H., Elliffe, D., Hunt, G. R., \& Gray, R. D. (2010). Complex cognition and behavioural innovation in New Caledonian crows. Proceedings of the Royal Society B: Biological Sciences, 277(1694), 2637-2643. doi:10.1098/rspb. 2010.0285

Taylor, A. H., Elliffe, D. M., Hunt, G. R., Emery, N. J., Clayton, N. S., \& Gray, R. D. (2011). New Caledonian crows learn the functional properties of novel tool types. PLOS ONE, 6(12). doi:10.1371/journal.pone.0026887

Taylor, A. H., Hunt, G. R., \& Gray, R. D. (2012). Context-dependent tool use in New Caledonian crows. Biology Letters, 8(2), 205-7. doi:10.1098/rsbl.2011.0782

Taylor, A. H., Hunt, G. R., Holzhaider, J. C., \& Gray, R. D. (2007). Spontaneous metatool use by New Caledonian crows. Current Biology, 17(17), 1504-1507. doi:10.1016/j.cub.2007.07.057 
Taylor, A. H., Hunt, G. R., Medina, F. S., \& Gray, R. D. (2009). Do New Caledonian crows solve physical problems through causal reasoning? Proceedings of the Royal Society B: Biological Sciences, 276(1655), 247-254. doi:10.1098/rspb. 2008.1107

Taylor, S. S., Boessenkool, S., \& Jamieson, I. G. (2008). Genetic monogamy in two long-lived New Zealand passerines. Journal of Avian Biology, 39(5), 579-583.

Taylor, S. S., Jamieson, I. G., \& Armstrong, D. P. (2005). Successful island reintroductions of New Zealand robins and saddlebacks with small numbers of founders. Animal Conservation, 8(4).

Terrace, H. S. (1987). Chunking by a pigeon in a serial learning task. Nature, 325(7000), 149-151. doi:10.1038/325149a0

Teufel, C., Alexis, D. M., Todd, H., Lawrance-Owen, A. J., Clayton, N. S., \& Davis, G. (2009). Social cognition modulates the sensory coding of observed gaze direction. Current Biology, 19(15), 1274-1277.

Tomasello, M., Call, J., \& Hare, B. (2003). Chimpanzees understand psychological states - the question is which ones and to what extent. Trends in Cognitive Sciences, 7(4), 153-156.

Tomonaga, M. (2008). Relative numerosity discrimination by chimpanzees (Pan troglodytes): Evidence for approximate numerical representations. Animal Cognition, 11(1), 43-57. doi:10.1007/s10071-007-0089-0

Townsend, A. K., Clark, A. B., McGowan, K. J., \& Lovette, I. J. (2009). Reproductive partitioning and the assumptions of reproductive skew models in the cooperatively breeding American crow. Animal Behaviour, 77(2), 503-512.

Trick, L. M., \& Pylyshyn, Z. W. (1994). Why are small and large numbers enumerated differently? A limited-capacity preattentive stage in vision. Psychological Review, 10(1), 80-102.

Udell, M. A., Dorey, N. R., \& Wynne, C. D. (2008). Wolves outperform dogs in following human social cues. Animal Behaviour, 76(6), 1767-1773. doi: 10.1016/j.anbehav.2008.07.028

Uller, C., Hauser, M. D., \& Carey, S. (2001). Spontaneous representation of number in cotton-top tamarins (Saguinus oedipus). Journal of Comparative Psychology, 115(3), 248-257.

Uller, C., Jaeger, R., Guidry, G., \& Martin, C. (2003). Salamanders (Plethodon cinereus) go for more: Rudiments of number in an amphibian. Animal Cognition, 6(2), 105-112.

Valenza, E., Leo, I., Gava, L., \& Simion, F. (2006). Perceptual completion in newborn human infants. Child Development, 77(6), 1810-1821. 
Vallortigara, G., \& Regolin, L. (2006). Gravity bias in the interpretation of biological motion by inexperienced chicks. Current Biology, 16(8), 279.

Vallortigara, G., Regolin, L., \& Marconato, F. (2005). Visually inexperienced chicks exhibit spontaneous preference for biological motion patterns. PLoS Biology, 3(7), e208.

Vander Wall, S. B. (1990). Food hoarding in animals. Chicago, IL, US: University of Chicago Press.

Vander Wall, S. B., \& Balda, R. P. (1981). Ecology and evolution of food-storage behaviour in conifer-seed-caching corvids. Zeitschrift Für Tierpsychologie, 56(3), 217-242.

Van Horik, J., \& Burns, K. C. (2007). Cache spacing patterns and reciprocal cache theft in New Zealand robins. Animal Behaviour, 73(6), 1043-1049.

vanMarle, K. L., \& Wynn, K. (2011). Tracking and quantifying objects and noncohesive substances. Developmental Science, 14(3), 502-515.

vanMarle, K. L., \& Wynn, K. (2006). Six-month-old infants use analog magnitudes to represent duration. Developmental Science, 9(5), F41-F49-F41-F49.

Von Bayern, A. M. P., \& Emery, N. J. (2009). Jackdaws respond to human attentional states and communicative cues in different contexts. Current Biology, 19(7), 602-606. doi:10.1016/j.cub.2009.02.062

von Fersen, L., \& Delius, J. D. (1989). Long-term retention of many visual patterns by pigeons. Ethology Formerly Zeitschrift Für Tierpsychologie, 82(2), 141-155.

von Fersen, L., \& Güntürkün, O. (1990). Visual memory lateralization in pigeons. Neuropsychologia, 28(1), 1-7.

Vonk, J., \& Subiaul, F. (2009). Do chimpanzees know what others can and cannot do? Reasoning about 'capability'. Animal Cognition, 267-286.

Watson, J. S. (1972). Smiling, cooing, and "the game". Merrill-Palmer Quarterly: Journal of Developmental Psychology, 18(4), 323-339.

Watve, M., Thakar, J., Kale, A., Puntambekar, S., Shaikh, I., Vaze, K., \& Paranjape, S. (2002). Bee-eaters (Merops orientalis) respond to what a predator can see. Animal Cognition, 5(4), 253-259.

West, R. E., \& Young, R. J. (2002). Do domestic dogs show any evidence of being able to count? Animal Cognition, 5(3), 183-186. doi:10.1007/ s10071-002-0140-0

Wheatley, T., Milleville, S. C., \& Martin, A. (2007). Understanding animate agents distinct roles for the social network and mirror system. Psychological Science, 18(6), 469-474. 
White, D. J., Ho, L., \& Freed-Brown, G. (2009). Counting chicks before they hatch: Female cowbirds can time readiness of a host nest for parasitism. Psychological Science, 20(9), 1140-1145-1140-1145.

Whitwell, S. M., Amiot, C., McLean, I. G., Lovegrove, T. G., Armstrong, D. P., Brunton, D. H., \& Ji, W. (2011). Losing anti-predatory behaviour: A cost of translocation. Austral Ecology. doi:10.1111/j.1442-9993.2011.02293.x

Woodward, A. L. (1999). Infants' ability to distinguish between purposeful and nonpurposeful behaviours. Infant Behaviour \& Development, 22(2), 145-160.

Wynn, K. (1992). Evidence against empiricist accounts of the origins of numerical knowledge. Mind \& Language, 7(4), 315-332.

Wynn, K. (1995). Origins of numerical knowledge. In B. Butterworth (Ed.), Mathematical cognition (Vol. 1, pp. 35-60).

Wynn, K., Cummins, D. D., \& Allen, C. (1998). An evolved capacity for number. In The evolution of mind (pp. 107-126). New York, NY, US: Oxford University Press.

Zucca, P., Milos, N., \& Vallortigara, G. (2007). Piagetian object permanence and its development in Eurasian jays (Garrulus glandarius). Animal Cognition, 10(2), 243-58. doi:10.1007/s10071-006-0063-2 